Preprint typeset in JHEP style - HYPER VERSION

ATL-PHYS-2004-029

Edinburgh 2004/12

\title{
Measurement of SUSY Masses via Cascade Decays for SPS 1a
}

\author{
B. K. Gjelsten \\ Department of Physics, University of Oslo, P.O.B. 1048, Blindern, N-0316 Oslo, \\ Norway \\ E-mail: B.K.Gjelsten@fys.uio.no \\ D. J. Miller \\ Department of Physics and Astronomy, University of Glasgow, Glasgow G12 8QQ, U.K. \\ and \\ School of Physics, University of Edinburgh, Edinburgh EH9 3JZ, U.K. \\ E-mail: D.Miller@physics.gla.ac.uk \\ P. Osland \\ Department of Physics, University of Bergen, N-5007 Bergen, Norway \\ E-mail: Per.0sland@ift.uib.no
}

ABSTRACT: If R-parity conserving supersymmetry exists below the TeV-scale, new particles will be produced and decay in cascades at the LHC. The lightest supersymmetric particle will escape the detectors, thereby complicating the full reconstruction of the decay chains. In this paper we expand on existing methods for determining the masses of the particles in the cascade from endpoints of kinematical distributions. We perform scans in the mSUGRA parameter space to delimit the region where this method is applicable. From the examination of theoretical distributions for a wide selection of mass scenarios it is found that caution must be exerted when equating the theoretical endpoints with the experimentally obtainable ones. We provide analytic formulae for the masses in terms of the endpoints most readily available. Complications due to the composite nature of the endpoint expressions are discussed in relation to the detailed analysis of two points on the SPS 1a line. Finally we demonstrate how a Linear Collider measurement can improve dramatically on the precision of the masses obtained.

KEYWORDS: SUSY, BSM, MSSM. 


\section{Contents}

1. Introduction 2

2. Cascade decays in mSUGRA scenarios

2.1 The mSUGRA mass hierarchy

2.2 Gluino and squark decays: the upper part of the chain

2.3 Neutralino and slepton decays: the lower part of the chain 10

2.4 Other constraints 12

3. Summary of SPS 1a 12

3.1 The SPS 1a line and points 12

3.2 Sparticle production 13

3.3 The cascade 16

4. Mass distributions 17

4.1 Theory curves of invariant mass distributions 18

4.2 Formulae for kinematic endpoints 19

4.3 Inversion formulae 21

5. 'Data' generation and reconstruction 24

5.1 Event generation 24

5.2 Different Flavour (DF) subtraction 25

5.3 Selection cuts 27

5.4 Multiple squark masses 30

5.5 Invariant mass distributions 31

6. Extraction of masses from edges 40

6.1 10,000 ATLAS experiments 40

6.2 Mass estimation via $\Sigma$

6.3 Minima of $\Sigma \quad 41$

6.4 SPS 1a $(\alpha) \quad 42$

6.5 SPS 1a $(\beta)$

7. Linear Collider inputs 50

8. Conclusions 51 


\section{Introduction}

The Standard Model (SM) of particle physics has been remarkably successful in describing the physics probed by modern day particle accelerators. No deviation from the SM has thus far been confirmed by experiment and only the Higgs mechanism, the SM's instrument for the breaking of the electroweak symmetry, remains to be discovered. Nevertheless, the SM suffers from considerable theoretical difficulties, not least of which is the hierarchy problem [1], the extreme sensitivity of the electroweak scale to new physics. Such difficulties imply that the SM is only an effective low-energy theory (albeit a highly successful one) applicable only up to a few hundred $\mathrm{GeV}$ or so, and will need to be extended in order to describe physics at higher scales.

One extension which has attracted a lot of attention is supersymmetry [2, 3, 4, 5]. Supersymmetry not only solves the hierarchy problem but has many other attractive features: it is the only non-trivial extension to the Poincaré symmetry of space-time [6]; it is essential to the formulation of superstring theories [7]; it provides a low-energy theory which is more amenable to the unification of the fundamental forces into a Grand Unified Theory (GUT) at some high energy scale [8]; it provides a natural mechanism for generating the Higgs potential which breaks the electroweak symmetry [9, 10, 11, 12, 13, 14]; and it supplies a good candidate for cold dark matter [15]. Furthermore, if it is to be relevant in solving the hierarchy problem it must exhibit experimental consequences at the $\mathrm{TeV}$-scale, and therefore can be tested by experiment at the Large Hadron Collider (LHC). For an overview of supersymmetry searches at LEP, the Tevatron and HERA, see Ref. [16].

If supersymmetric particles are produced at the LHC, thus confirming supersymmetry, it will become important to identify them and accurately measure their masses. This will be essential for identifying the low energy model and hopefully distinguishing the Minimal Supersymmetric Standard Model (MSSM) from other non-minimal extensions. Furthermore, since no supersymmetric particles have so far been discovered, supersymmetry must be broken by some as yet unknown mechanism. Only an accurate determination of the supersymmetric particle masses and couplings will allow us to determine the low energy soft supersymmetry breaking parameters. It is hoped that extrapolation of these masses and couplings to high energies using the renormalisation group equations will provide an insight into the mechanism of supersymmetry breaking and, more generally, physics at the GUT scale [17. Since errors in the mass measurements will be magnified by the renormalisation group running it is absolutely essential that these masses be determined as accurately as possible.

Here we will discuss supersymmetric mass measurements with reference to one particu-

lar model of supersymmetry breaking, minimal super-gravity (mSUGRA) [18, 12, 9, 13, 14]. In this model, the supersymmetry is broken by the interaction of new particles at high energy which are only linked to the usual particles by gravitational interactions; this new sector of physics is often referred to as the hidden sector. These gravitational interactions transmit the supersymmetry breaking from the hidden sector to our own sector, producing TeV scale effective soft supersymmetry breaking terms in the GUT scale Lagrangian, quantified by parameters which run logarithmically down to the probed $\mathrm{TeV}$ scale. At the 
GUT scale, the scalar supersymmetric particles are assumed to have a common mass, $m_{0}$, while the gauginos have a common mass $m_{1 / 2}$. The trilinear couplings are also taken to be universal at the GUT scale and denoted $A_{0}$.

Mass measurements in the MSSM are complicated by R-parity conservation, which is introduced to prevent unphysical proton decay. R-parity conservation requires that supersymmetric particles are produced in pairs and causes the lightest supersymmetric particle (LSP) to be stable. Consequently the LSP is inevitably the end product of every supersymmetric decay and, if electrically neutral, will escape the detector leaving no track or energy deposit. While this provides a very distinctive missing energy signature, it makes it very difficult to measure masses at the LHC since one cannot fully reconstruct decays.

Instead, mass measurements rely on continuous mass distributions of decay products which attain extrema for certain configurations of the particle momenta that are unambiguously determined by the masses of initial, intermediate and final particles involved. These relations may often be inverted to give the masses of unstable particles. This is analogous to the way a bound on the neutrino mass can be obtained from the end-point of the beta-decay spectrum of ${ }^{3} \mathrm{H}$ [19], but is usually more complex, since a long decay chain is often involved.

In this study we will consider supersymmetric mass measurements made by examining the mass distribution endpoints or 'edges' of the long decay chain ${ }^{1} \tilde{q} \rightarrow \tilde{\chi}_{2}^{0} q \rightarrow \tilde{l} l q \rightarrow \tilde{\chi}_{1}^{0} l l q$ in the ATLAS detector [20]. In particular, we will focus on the Snowmass mSUGRA benchmark line SPS 1a [21], but will also include other mSUGRA parameters in a general discussion. In addition to the usual SPS 1a point, which we will denote SPS 1a $(\alpha)$, we will also consider another point on the SPS 1a line, denoted SPS 1a $(\beta)$, which has a reduced branching ratio for the decay. This will provide a counterpoint to the study of SPS 1a $(\alpha)$ where the branching ratio is rather high. This study differs from previous reports 22, 23, 24, 25, 26] by (i) discussing theoretical distributions which arise for different mass scenarios, (ii) providing inversion formulas, (iii) discussing ambiguities and complications related to the composite nature of the endpoint expressions. Furthermore, we provide an overview of the mSUGRA parameter space, and consider a new point on the SPS 1a line. Finally, we discuss the effects of including Linear Collider data in the analysis.

In Sect. 2 we will discuss general mSUGRA scenarios, paying attention to supersymmetric decay branching ratios to gain some understanding of how generally applicable these kinematic endpoint measurements are. We will identify mass hierarchies for which these mass measurements are possible and show that they occur over a large portion of the mSUGRA parameter space. In Sect. 3 we will outline the properties and mass spectra of the Snowmass benchmark line SPS 1a and the associated points SPS 1a $(\alpha)$ and SPS 1a $(\beta)$. After defining the decay chain under investigation, we go on in Sect. 4 to discuss the theoretical framework of cascade endpoint measurements, and present analytic expressions for masses in terms of these endpoints. In Sect. 5 the experimental situation at the ATLAS detector will be studied, including event generation and reconstruction, the removal of backgrounds, and results for the measurement of the kinematic endpoints will

\footnotetext{
${ }^{1}$ Throughout the text the notions lepton/slepton as well as $l / \tilde{l}$ will refer to the particles of the first and second generation. The third generation particles will be called tau/stau and denoted $\tau / \tilde{\tau}$.
} 
be presented. The extraction of masses from the kinematic endpoints will be described in Sect. 6. Finally, the remarkable improvement of the accuracy of the mass measurements obtained by using inputs from an $e^{+} e^{-}$collider [27, 28] will be studied in Sect. 7, before drawing our conclusions in Sect. 8 .

\section{Cascade decays in mSUGRA scenarios}

In this paper we will be examining the decay chain $\tilde{q} \rightarrow \tilde{\chi}_{2}^{0} q \rightarrow \tilde{l} l q \rightarrow \tilde{\chi}_{1}^{0} l l q$ in the Snowmass scenario SPS 1a for the purpose of the extraction of the supersymmetric particle masses. However, it is extremely unlikely that SPS 1a is exactly the parameter choice of reality, and there would be little point to the study if our methods were only applicable at SPS 1a, or a small region around it. Therefore, in this section we will take a more general look at mSUGRA scenarios to determine whether or not these methods may be used more generally, over a wide parameter range.

At the LHC the main supersymmetric production will be sparticle pairs $\tilde{g} \tilde{g}, \tilde{g} \tilde{q}$ and $\tilde{q} \tilde{q}$, as long as these are not too heavy. Each sparticle immediately decays into a lighter sparticle, which in turn decays further, until, at the end of the chain, an LSP will be produced. Since there are two parent supersymmetric particles, each event will typically have two such chains, complicating their reconstruction.

What route is taken from the initial gluino or squark down to the LSP depends on which decay channels are open, as well as their branching fractions. Therefore there are two criteria which must be met in order to use the cascade kinematic endpoint methods: firstly the sparticle mass hierarchy must be such that the analysed decay chain is allowed; and secondly, the cross-section for the entire decay chain must be large enough to allow analysis.

\subsection{The mSUGRA mass hierarchy}

In a general MSSM model there are few constraints on the sparticle masses, so little can be assumed about their relative mass hierarchy. However, if universal boundary conditions are imposed on the mass parameters at the GUT scale, some mass orderings at the $\mathrm{TeV}$ scale are natural, and some are even necessary.

For example, in mSUGRA scenarios squarks and sleptons have a common mass at the GUT scale, $m_{0}$, but when the masses are evolved down to the TeV scale, QCD interactions affect only the evolution of the squarks and not the colourless sleptons. Consequently, squarks are always heavier than sleptons at LHC energies. For the same reasons, although they may start off with different masses at the GUT scale, running induced by QCD interactions is usually enough to result in the squarks of the first two generations being heavier than the neutralinos and charginos at the $\mathrm{TeV}$ scale. (Large mixing can however bring $\tilde{t}_{1}$, and to a lesser extent $\tilde{b}_{1}$, quite low in mass.)

At the opposite end of the spectrum, there are a number of possible candidates for

the LSP: the lightest neutralino, $\tilde{\chi}_{1}^{0}$, the lightest chargino $\tilde{\chi}_{1}^{ \pm}$, or the lightest slepton (in mSUGRA models, the gravitino is usually rather heavy). Which of these is the LSP depends on the relative sizes of $m_{0}, m_{1 / 2}$ and the derived low-energy Higgs-higgsino mass 
parameter $\mu$. However, the assumption of gauge unification at the GUT scale explicit in mSUGRA models leads to the relation

$$
M_{1} \approx \frac{5}{3} \tan ^{2} \theta_{W} M_{2}
$$

between the $U(1)$ and $S U(2)$ gaugino masses, $M_{1}$ and $M_{2}$ respectively. As a result, $M_{1}$ tends to be rather low, significantly lower than $m_{1 / 2}$. Furthermore, the derived quantity $\mu$ is often required to be much larger than $M_{1}$ in order to give the correct electroweak symmetry breaking (at the SPS $1 \mathrm{a}(\alpha)$ reference point $\mu=357.4 \mathrm{GeV}$ ). For the majority of parameter choices this implies that the LSP will be $\tilde{\chi}_{1}^{0}$, with $\tilde{\tau}_{1}$ being the LSP only if $m_{0} \ll m_{1 / 2}$, and $\tilde{\chi}_{1}^{ \pm}$only for a small region where $m_{1 / 2} \rightarrow 0$. The left-handed sneutrino, by virtue of its $S U(2)$ interactions, is usually heavier than $\tilde{\tau}_{1}$, and is anyway ruled out by direct searches [29]. It is indeed fortunate that $\tilde{\chi}_{1}^{0}$ is the LSP for most of the parameter space since it is clear that only an electrically neutral LSP can play the role of the dark matter constituent which is believed to fill the universe. Finally, the gaugino mass relation, Eq. (2.1), implies that the LSP is usually bino-like.

The first requirement for the decay chain $\tilde{q} \rightarrow \tilde{\chi}_{2}^{0} q \rightarrow \tilde{l} l q \rightarrow \tilde{\chi}_{1}^{0} l l q$ is that the gluino should be comparable to or heavier than the squark initiating the decay chain. If the gluino is sufficiently light, then the squark will almost always choose to decay via its strong interaction $\tilde{q} \rightarrow \tilde{g} q$ rather than by the electroweak decay $\tilde{q} \rightarrow \tilde{\chi}_{2}^{0} q$. Of course, one does not need all of the squarks to be lighter than the gluino; as long as one squark, for example $\tilde{b}_{1}$, is lighter than the gluino, useful information can potentially be obtained from its subsequent decay chain. The second important characteristic is that $\tilde{\chi}_{2}^{0}$ should be heavier than $\tilde{l}$, thereby allowing the lower part of the chain to proceed, $\tilde{\chi}_{2}^{0} \rightarrow \tilde{l} l \rightarrow \tilde{\chi}_{1}^{0} l l$. Otherwise $\tilde{\chi}_{2}^{0}$ will decay to $\tilde{\chi}_{1}^{0} Z$ or $\tilde{\chi}_{1}^{0} h$, or to $\tilde{\chi}_{1}^{0} f \bar{f}$ via a three-body decay, and the useful kinematic endpoints are lost.

In order to understand where in the mSUGRA parameter space these hierarchy requirements are realised, we have performed a scan over the $m_{1 / 2}-m_{0}$ plane for four different choices of $A_{0}$ and $\tan \beta$ (with $\mu>0$ ), and identified the different hierarchy regions with different colours in Fig. 1. The renormalisation group running of the parameters from the GUT scale to the TeV scale has been done using version 7.58 of the program ISAJET [30], which is inherent to the definition of the 'Snowmass Points and Slopes' (see Sect. 3).

The upper left plot shows the $m_{1 / 2}-m_{0}$ plane with $A_{0}=-m_{0}$ and $\tan \beta=10$ and includes the SPS 1a line and points (labeled $(\alpha)$ and $(\beta)$ ). The upper right plot has $A_{0}=0$ and $\tan \beta=30$ and contains the benchmark point SPS 1b. The lower left plot also has $A_{0}=0$ but $\tan \beta=10$ and contains the SPS 3 benchmark line and point. Finally the lower right plot has $A_{0}=-1000 \mathrm{GeV}$ and $\tan \beta=5$ and contains SPS 5 .

The different hierarchies themselves are combinations of the hierarchy between the gluino and the squarks important to the upper part of the decay chain, and that of $\tilde{\chi}_{2}^{0}$ and the sleptons relevant to the later decays. Since $m_{\tilde{l}_{R}}<m_{\tilde{l}_{L}}$ for any set of mSUGRA parameters, we here use $\tilde{l}_{R}$. The seven numbered regions are defined by:

$$
\text { (i) } \quad \tilde{g}>\max \left(\tilde{d}_{L}, \tilde{u}_{L}, \tilde{b}_{1}, \tilde{t}_{1}\right) \quad \text { and } \quad \tilde{\chi}_{2}^{0}>\max \left(\tilde{l}_{R}, \tilde{\tau}_{1}\right)
$$



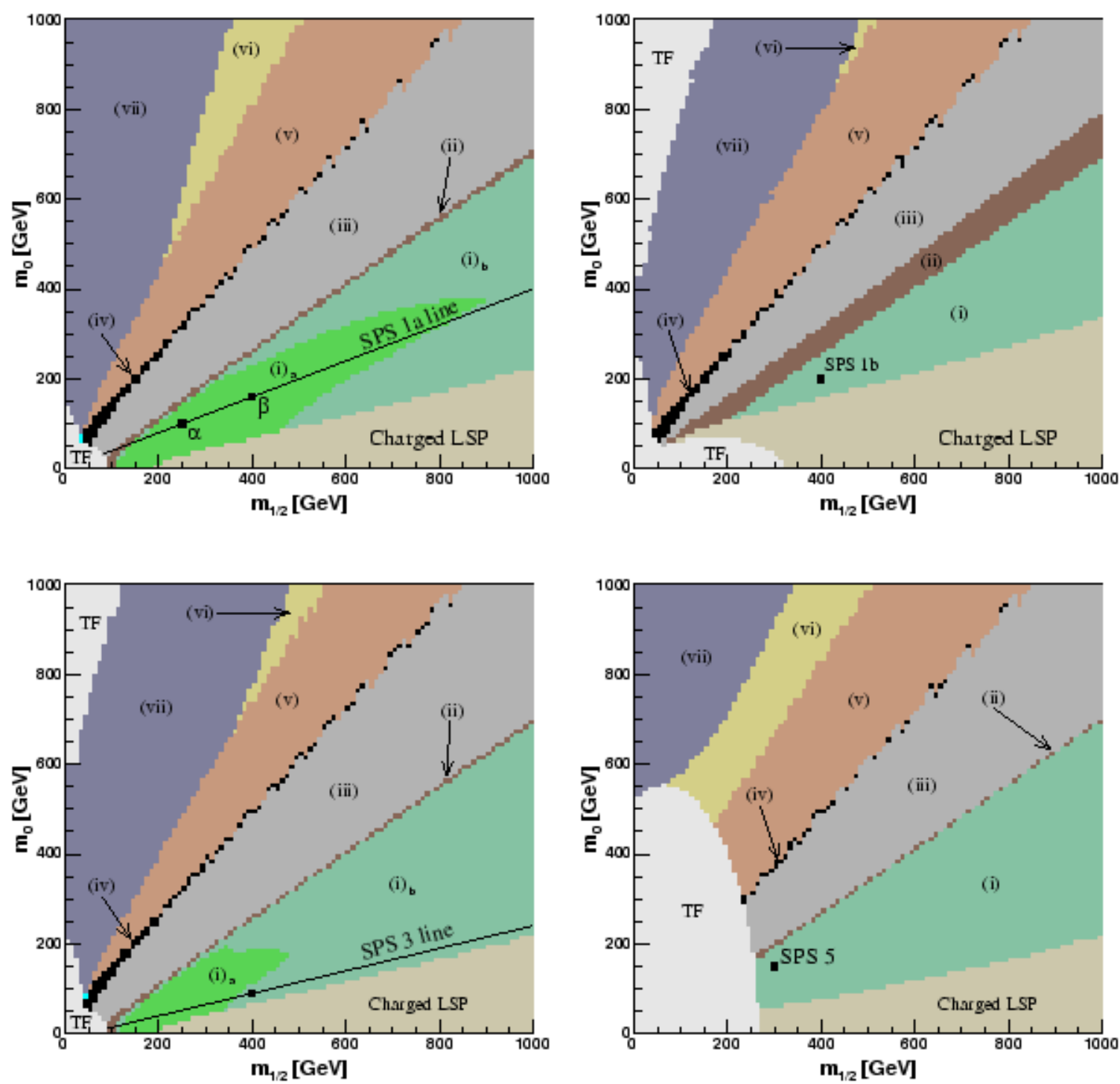

Figure 1: Classification of different hierarchies, labeled (i)-(vii), for four combinations of $\tan \beta$ and $A_{0}$, such that the four panels contain respectively the SPS 1a line, the SPS 1b point, the SPS 3 line, and the SPS 5 point. The regions marked 'TF' are theoretically forbidden. (See text for details.)

$$
\begin{array}{llll}
\text { (ii) } & \tilde{g}>\max \left(\tilde{d}_{L}, \tilde{u}_{L}, \tilde{b}_{1}, \tilde{t}_{1}\right) & \text { and } & \tilde{l}_{R}>\tilde{\chi}_{2}^{0}>\tilde{\tau}_{1} \\
\text { (iii) } & \tilde{g}>\max \left(\tilde{d}_{L}, \tilde{u}_{L}, \tilde{b}_{1}, \tilde{t}_{1}\right) & \text { and } & \min \left(\tilde{l}_{R}, \tilde{\tau}_{1}\right)>\tilde{\chi}_{2}^{0} \\
\text { (iv) } & \tilde{d}_{L}>\tilde{g}>\max \left(\tilde{u}_{L}, \tilde{b}_{1}\right) & \text { and } & \min \left(\tilde{l}_{R}, \tilde{\tau}_{1}\right)>\tilde{\chi}_{2}^{0} \\
\text { (v) } & \min \left(\tilde{d}_{L}, \tilde{u}_{L}\right)>\tilde{g}>\tilde{b}_{1} & \text { and } & \min \left(\tilde{l}_{R}, \tilde{\tau}_{1}\right)>\tilde{\chi}_{2}^{0} \\
\text { (vi) } & \min \left(\tilde{d}_{L}, \tilde{u}_{L}, \tilde{b}_{1}\right)>\tilde{g}>\tilde{t}_{1} & \text { and } & \min \left(\tilde{l}_{R}, \tilde{\tau}_{1}\right)>\tilde{\chi}_{2}^{0} \\
\text { (vii) } & \min \left(\tilde{d}_{L}, \tilde{u}_{L}, \tilde{b}_{1}, \tilde{t}_{1}\right)>\tilde{g} & \text { and } & \min \left(\tilde{l}_{R}, \tilde{\tau}_{1}\right)>\tilde{\chi}_{2}^{0}
\end{array}
$$

where for fermions a particle's symbol represents its mass, while for scalars a particle's sym- 
bol represents the sum of the masses of the scalar and its SM partner. Also shown (mauve) is a region where the LSP is charged and therefore ruled out, as well as a theoretically forbidden (TF) region (gray) for low $m_{1 / 2}$.

It is interesting to note that there are no regions where a squark is heavier than the gluino and $\tilde{\chi}_{2}^{0}$ is heavier than one of the sleptons. This is simply because the gluino and [the gaugino part of] the neutralino have a common mass, $m_{1 / 2}$, at the high scale, so if the gluino is light, the neutralinos will also be light.

However, for more general non-mSUGRA unification scenarios one could still expect hierarchies of the type $m_{\tilde{q}_{L}}>m_{\tilde{g}}>m_{\tilde{\chi}_{2}^{0}}>m_{\tilde{l}}>m_{\tilde{\chi}_{1}^{0}}$ to be realised. It would then be important to be able to distinguish one hierarchy from the other; this should be possible using the kinematic endpoints, number of $b$-quarks in the final state, etc. Also it is possible to distinguish $\tilde{\chi}_{2}^{0} \rightarrow \tilde{l} l \rightarrow \tilde{\chi}_{1}^{0} l l\left(m_{\tilde{\chi}_{2}^{0}}>m_{\tilde{l}}\right)$ from $\tilde{\chi}_{2}^{0} \rightarrow \tilde{\chi}_{1}^{0} l l\left(m_{\tilde{l}}>m_{\tilde{\chi}_{2}^{0}}\right)$. The first has the well-known triangular shape of $m_{l l}$ while the second has a typical 3-body shape. All in all it should therefore be possible to distinguish the various hierarchies (2.2) before continuing to determine the masses themselves.

Region (i) is the only one that has a 'useful' squark decay together with a decay of $\tilde{\chi}_{2}^{0}$ to a slepton, and is shown in light and dark green in Fig. 1. We see that there is therefore a large region where the mass hierarchy is compatible with the methods presented here, and even though we will only perform the analysis for the points $(\alpha)$ and $(\beta)$ on the SPS 1a line, one would expect these methods to be widely applicable.

However, these plots of the mass hierarchies really only show the regions in which the masses are such that the decay chain may occur. If the full decay chain is to be useful, it must have a sufficiently large branching ratio to be seen above the many backgrounds. We will therefore go on to examine the branching ratios of the pertinent decays over (a restricted range of) the mSUGRA parameter space. As a first taste, we have highlighted in a brighter green (and denoted $(\mathrm{i})_{a}$ ) the part of region (i) corresponding to where the overall branching ratio for the decay chain $\tilde{q} \rightarrow \tilde{\chi}_{2}^{0} q \rightarrow \tilde{l} l q \rightarrow \tilde{\chi}_{1}^{0} l l q$ exceeds a tenth of that at the SPS 1a $(\alpha)$ reference point. Although the decay chain is available over a rather large region of the parameter space, using this decay for large values of $m_{1 / 2}$ and $m_{0}$ will be extremely challenging due to the small branching ratio.

\subsection{Gluino and squark decays: the upper part of the chain}

The decay branching ratios of the gluino are shown in Fig. 2 over the $m_{1 / 2}-m_{0}$ plane for two different scenarios. The representation is such that the branching ratio of a given decay channel in a small neighbourhood of the $m_{1 / 2}-m_{0}$ plane is equal to the fraction which the corresponding colour occupies in that neighbourhood. Since the gluino only feels the strong force, it has to decay into a quark and a squark. If no squark is light enough, a three-body decay through an off-shell squark will take place; this is what happens in the green/white region of Fig. 20 at small $m_{1 / 2}$.

For the rest of the $m_{1 / 2}-m_{0}$ plane the gluino decays fairly democratically into the

accessible squarks. In considerable parts of the plane only one two-body decay is open, $\tilde{b}_{1} b$ (red) or $\tilde{t}_{1} t$ (yellow), in which case the allowed decay takes close to the full decay width. Although one can in principle obtain information about the gluino mass by analysing its 

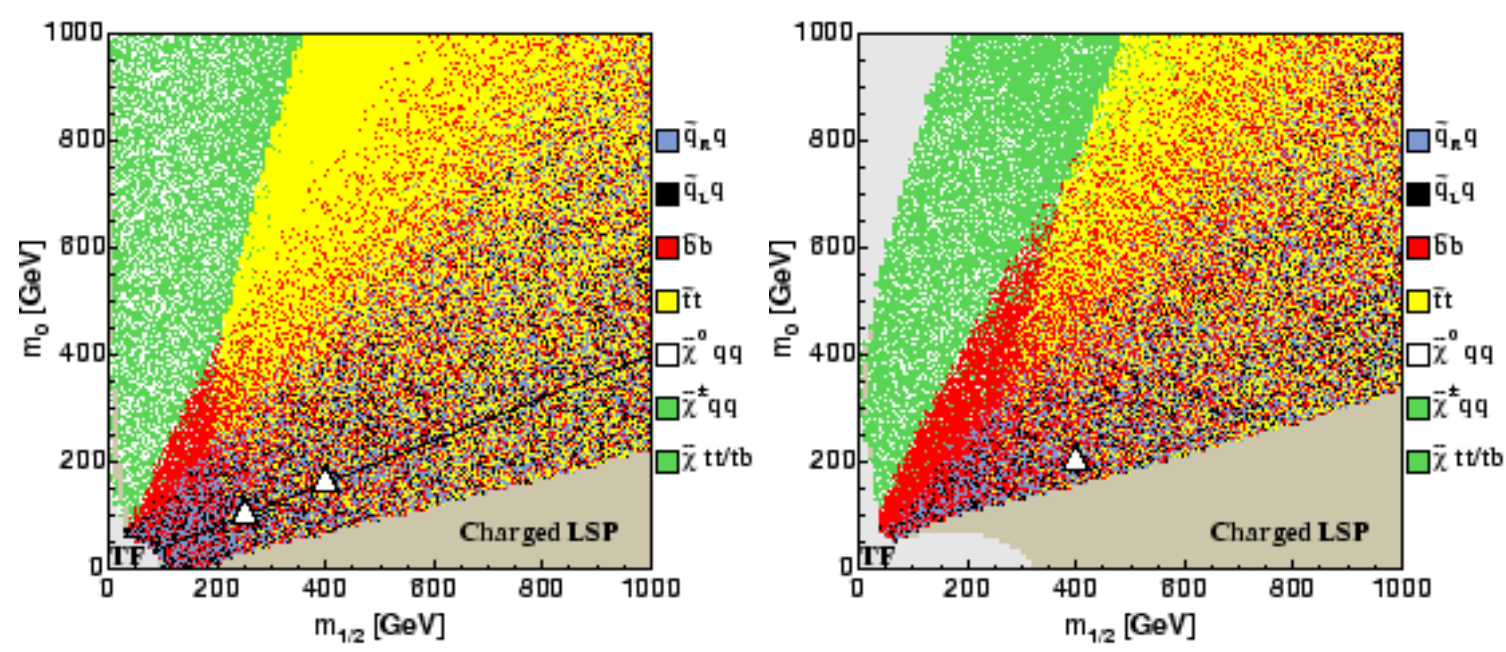

Figure 2: Decay channels of $\tilde{g}$ with $A_{0}=-m_{0}, \tan \beta=10$ (left) and $A_{0}=0, \tan \beta=30$ (right). In the left panel the SPS 1a line is shown together with the two points $(\alpha)$ and $(\beta)$ marked with triangles. In the right panel the triangle marks the SPS 1b point. The branching ratio of a given decay channel in a small neighbourhood of the $m_{1 / 2}-m_{0}$ plane is equal to the fraction which the corresponding colour occupies in that neighbourhood. The region where $\tilde{\chi}_{1}^{0}$ is not the LSP is denoted 'Charged LSP' and is discarded. Some regions are also forbidden theoretically, in that e.g. it is not possible to obtain electroweak symmetry breaking (labeled 'TF').

decay chain, we will only consider here the decay chain starting from a parent squark, and leave the gluino case for a separate publication [33].

As already intimated, squarks may decay by the strong force into a quark and a gluino (if the gluino is lighter), or decay by weak interactions into a quark and a chargino or neutralino, or via a loop into a gluon and a lighter squark. If kinematically allowed, the strong interaction takes a large fraction of the branching ratio, but since the (lighter) charginos and neutralinos are typically much lighter than the gluino, there will always be some neutralino production.

Within mSUGRA models, the squarks $\tilde{d}_{L}$ and $\tilde{u}_{L}$ are very close in mass and behaviour. Furthermore, the second generation squarks, $\tilde{s}_{L}$ and $\tilde{c}_{L}$, are almost identical copies of the former two. It is therefore useful to have the common notation, $\tilde{q}_{L}$, for these four squarks. In a similar manner $\tilde{q}_{R}$ is used for $\tilde{d}_{R}, \tilde{u}_{R}$ and their second generation copies. The righthanded squarks differ from the left-handed ones in that they do not feel weak interactions, which again makes their decay pattern different. In Fig. 3 the decay branching ratios of $\tilde{u}_{L}$ and $\tilde{u}_{R}$ are shown in the $m_{1 / 2}-m_{0}$ plane for two different scenarios.

For $m_{1 / 2} \ll m_{0}$, when the gluino mass is smaller than the squark mass, both $\tilde{q}_{L}$ and even more so $\tilde{q}_{R}$ have strong-interaction decays. In the rest of the $m_{1 / 2}-m_{0}$ plane, when the strong decay is forbidden or suppressed by phase space, their decay patterns are very different: while $\tilde{q}_{R}$ decays directly into the LSP, $\tilde{q}_{L}$ prefers $\tilde{\chi}_{2}^{0}$ and $\tilde{\chi}_{1}^{ \pm}$.

For both low and high $\tan \beta$, the $\tilde{\chi}_{1}^{0}$ is predominantly bino, with only a tiny admixture of wino and higgsino, while $\tilde{\chi}_{2}^{0}$ and $\tilde{\chi}_{1}^{ \pm}$are mainly wino. For quite low mass parameters, 

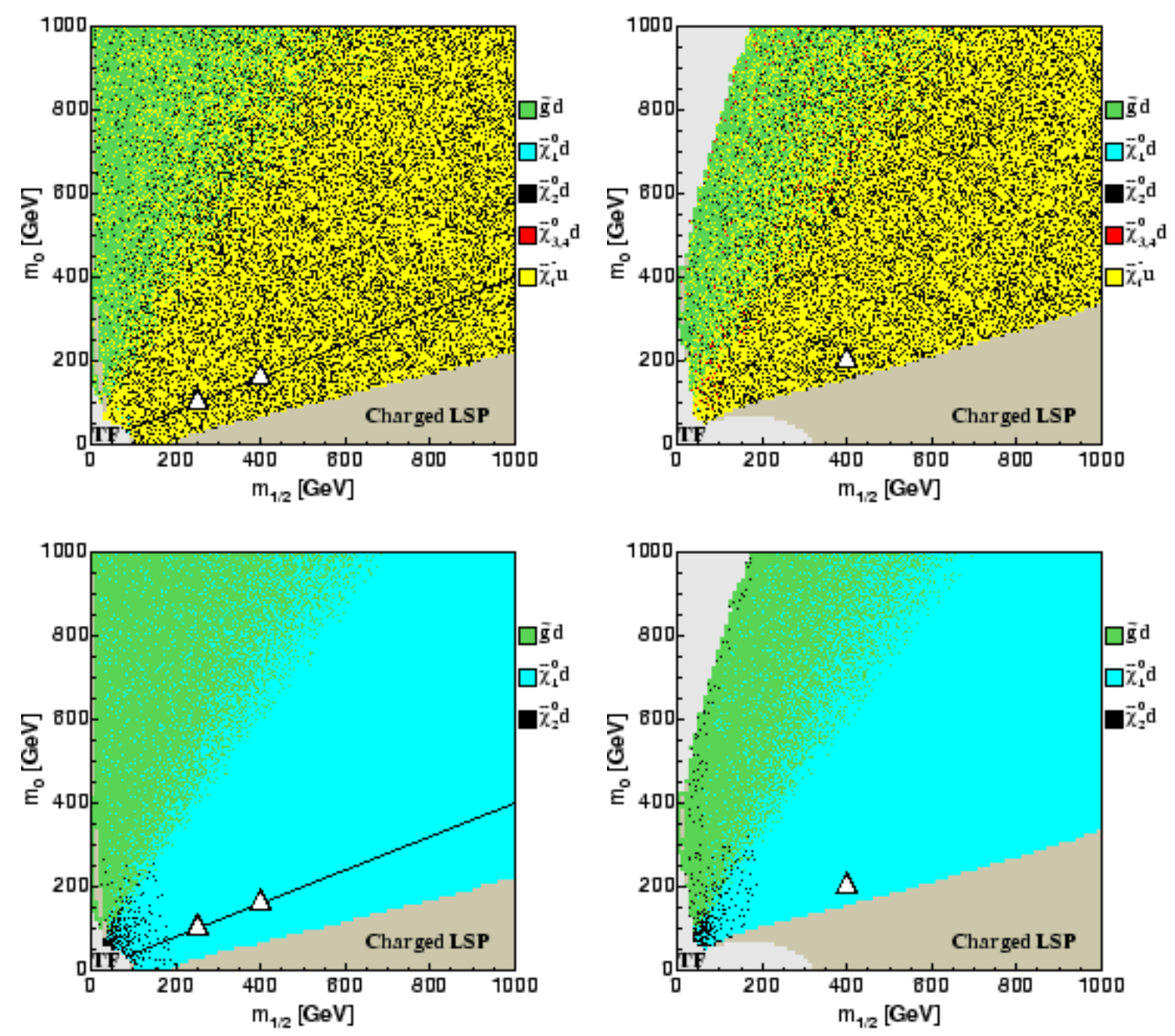

Figure 3: Decay channels of $\tilde{u}_{L}$ (top) and $\tilde{u}_{R}$ (bottom) with $A_{0}=-m_{0}, \tan \beta=10$ (left) and $A_{0}=0, \tan \beta=30$ (right). (See the caption of Fig. 2.) Note that the branching ratios for all first and second-generation squarks are assumed to be essentially the same.

$m_{1 / 2} \lesssim 100 \mathrm{GeV}$, they become more mixed. Since $\tilde{q}_{L}$ generally has a much larger $\mathrm{SU}(2)$ coupling than $\mathrm{U}(1)$ coupling, decays to $\tilde{\chi}_{1}^{ \pm}$and $\tilde{\chi}_{2}^{0}$ will be preferred unless the difference in phase space makes the decay to the lighter $\tilde{\chi}_{1}^{0}$ competitive. In contrast, since the $\tilde{q}_{R}$ has no $\mathrm{SU}(2)$ interaction it will decay predominantly to the bino $\tilde{\chi}_{1}^{0}$, except at quite low mass parameters where the neutralinos change character.

The third generation squarks differ from the others in two aspects. First, the mass eigenstates can have more even admixtures of both handedness components. For $\tilde{b}$ this is the case for low mass parameters, $m_{0}, m_{1 / 2} \lesssim 200 \mathrm{GeV}$, where the branching ratios into $\tilde{\chi}_{1}^{0}$ and $\tilde{\chi}_{2}^{0}$ are of comparable size. At higher masses $\tilde{b}_{1} \approx \tilde{b}_{L}$ and $\tilde{b}_{2} \approx \tilde{b}_{R}$, giving a $\tilde{b}_{1}$ which prefers to go to $\tilde{\chi}_{2}^{0}$ rather than $\tilde{\chi}_{1}^{0}$. Second, due to large splitting, the third generation squarks can decay into other third generation squarks together with a weak gauge boson. The drastic change observed in figure 1 as $m_{0}, m_{1 / 2}$ become less than $\sim 200 \mathrm{GeV}$, is due 

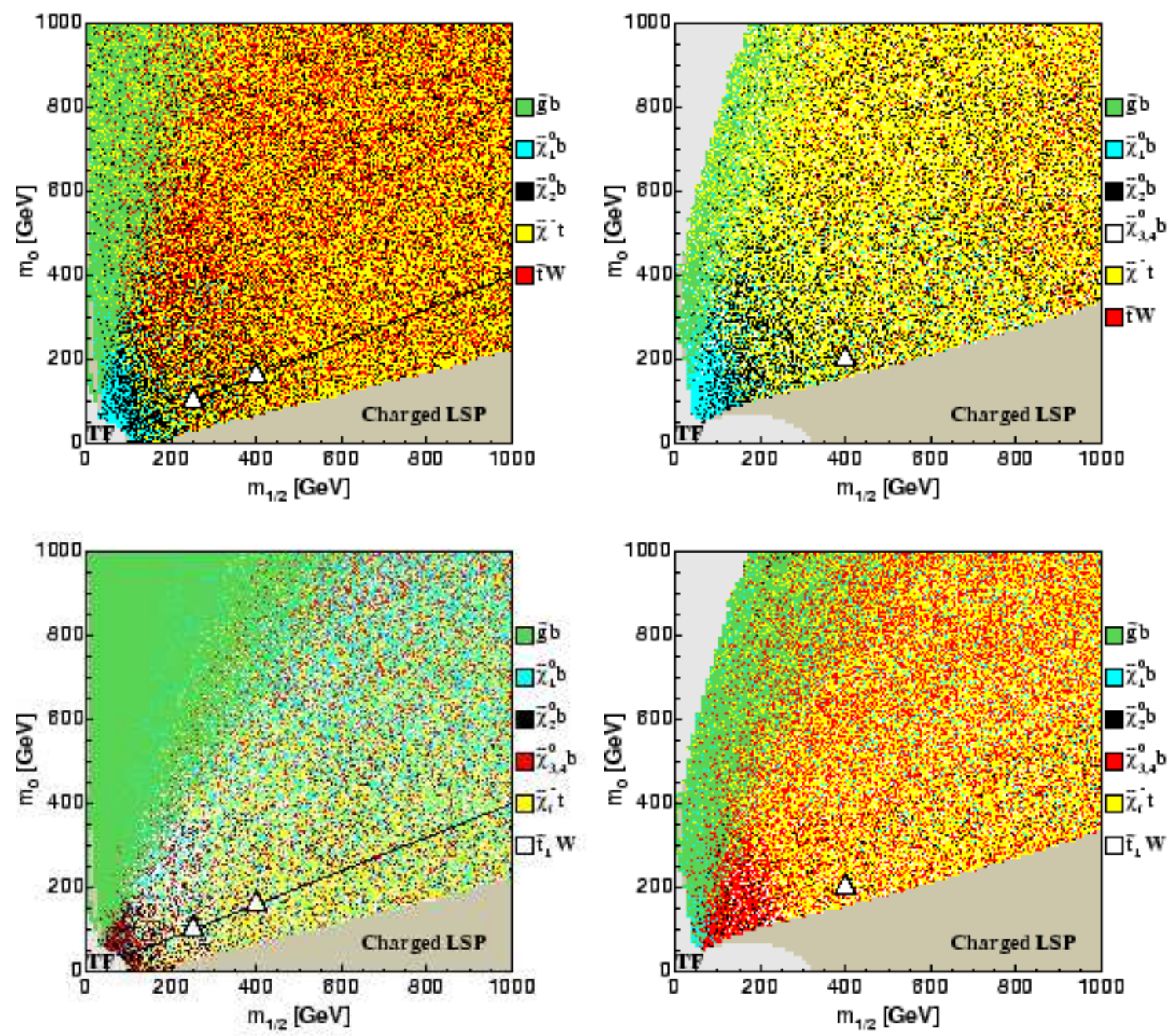

Figure 4: Decay channels of $\tilde{b}_{1}$ (upper panels) and $\tilde{b}_{2}$ (lower panels) with $A_{0}=-m_{0}, \tan \beta=10$ (left) and $A_{0}=0, \tan \beta=30$ (right). (See the caption of Fig. 2.)

both to the more mixed mass eigenstates for lower masses, and to the closing of certain channels involving $t$ or a heavy gauge boson.

While $\tilde{b}_{1}$ has a large branching ratio into $\tilde{\chi}_{2}^{0}$ throughout the entire plane, $\tilde{b}_{2}$ produces $\tilde{\chi}_{2}^{0}$ at a much smaller rate, except for small mass parameters.

To summarize, the squark decays that are 'useful' for kinematic endpoint analyses, are those of left-handed first and second-generation squarks, as well as those of $\tilde{b}_{1}$ and to a lesser extent $\tilde{b}_{2}$. These occur in the entire $m_{1 / 2}-m_{0}$ plane, except for extreme values $m_{1 / 2} \ll m_{0}$, and for both low and high $\tan \beta$ values. For quite low mass parameters also $\tilde{q}_{R}$ contributes.

\subsection{Neutralino and slepton decays: the lower part of the chain}

The reasons why $\tilde{\chi}_{2}^{0}$ often plays an important role in the reconstruction of SUSY events are many. Kinematically situated midway between the initially produced gluinos/squarks and 

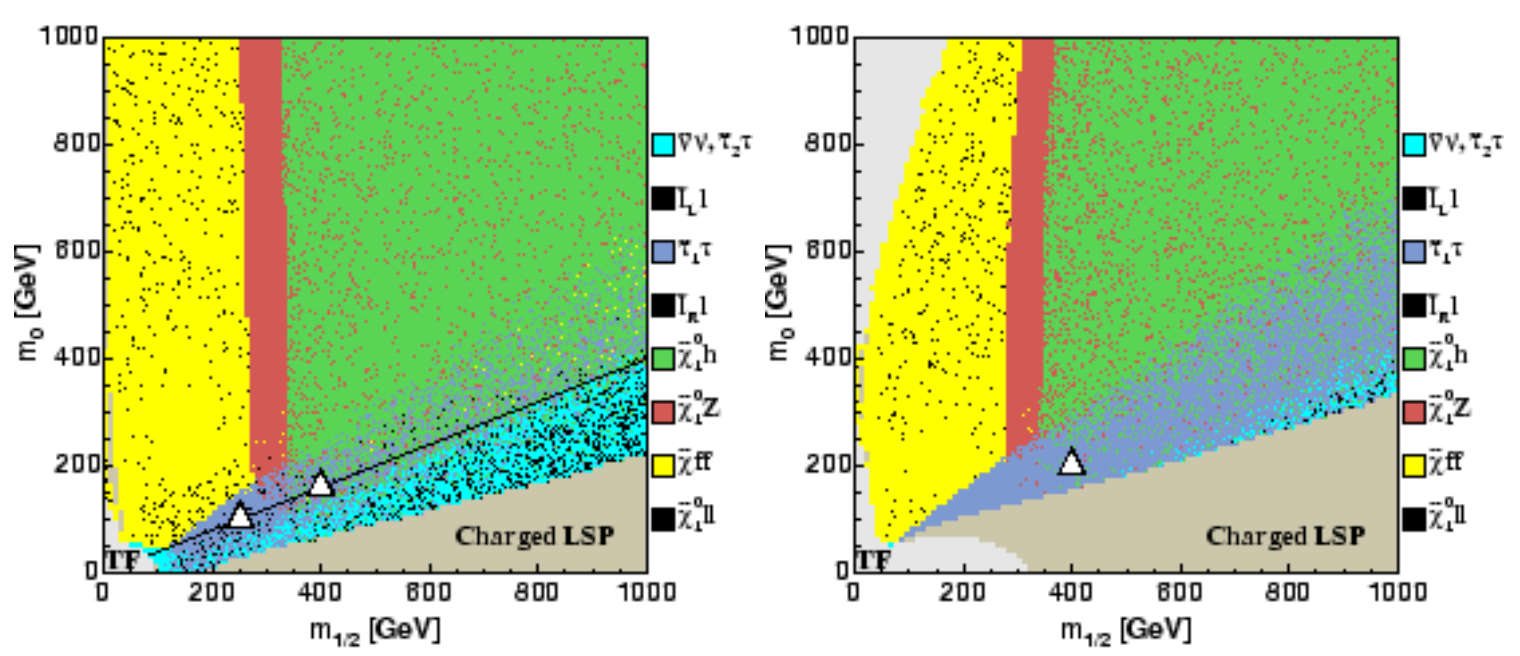

Figure 5: Decay channels of $\tilde{\chi}_{2}^{0}$ with $A_{0}=-m_{0}, \tan \beta=10$ (left) and $A_{0}=0, \tan \beta=30$ (right). (See the caption of Fig. 2.)

the LSP, it is abundantly decayed into, as we have seen. What makes it so useful, usually more so than the $\tilde{\chi}_{1}^{ \pm}$, which is produced in similar ways and amounts, is the fact that its decay products, in addition to easily setting off the trigger, also reconstruct well.

In Fig. 5 the main decay channels of $\tilde{\chi}_{2}^{0}$ are shown for two values of $\tan \beta$. A two-body decay is preferred over a three-body decay, and the coupling to $\tilde{l}, \tilde{\tau}$ or $\tilde{\nu}$ is usually stronger than the coupling to $\tilde{\chi}_{1}^{0}$.

For $m_{0} \gtrsim 0.5 m_{1 / 2}$ all of $\tilde{l}, \tilde{\tau}$ and $\tilde{\nu}$ are heavier than $\tilde{\chi}_{2}^{0}$, so only the decay into the LSP is possible. In yellow, to the very left, no two-body channel is open, and $\tilde{\chi}_{2}^{0}$ undergoes a three-body decay, proceeding through an off-shell squark or stau/slepton, or involving an off-shell $Z, W$ or $h$. As $m_{1 / 2}$ increases, also $m_{\tilde{\chi}_{2}^{0}}-m_{\tilde{\chi}_{1}^{0}}$ increases and more decay channels become available. First the $Z$ channel opens and takes the full decay width, then the $h$ channel opens to dominate. The mass difference between $\tilde{\chi}_{1}^{0}$ and $\tilde{\chi}_{2}^{0}$ is mostly independent of $m_{0}$, which is why, to a good approximation, the yellow, red and green regions are stacked horizontally.

In the blue regions decays into $\tilde{l} / \tilde{\tau} / \tilde{\nu}$ are kinematically allowed. Following a clockwise movement, the scalar masses are reduced relative to $\tilde{\chi}_{2}^{0}$. The right-handed scalars are lighter and become available first (dark blue region). In the light blue region the lefthanded scalars have become available and, despite less phase space, take most of the width due to their $\mathrm{SU}(2)$ coupling. The black part of the blue regions shows the decay into $\tilde{l}_{R}$ (dark blue region) and $\tilde{l}_{L}$ (light blue region). These are the decays of interest to us. At low $\tan \beta$ (left panel) the slepton channels can be used in most of the blue regions. At high $\tan \beta$ the situation is less optimistic. The $\tilde{\tau}_{1}$ channel totally dominates the $\tilde{l}_{R}$ channel, and only in a small region is the $\tilde{l}_{L}$ channel open.

Decay products which involve tau particles are more difficult to use since their reconstruction is always incomplete due to undetected neutrinos. However, in some parts of the parameter space, especially at high $\tan \beta$, these channels take the full decay width, so one 
must be prepared to use them. In any case, whatever the MSSM parameters should turn out to be, in order to measure the stau mass itself, tau particles must be reconstructed.

In the non-blue regions of the plane, information on the sparticle masses can still be retrieved, although to a lesser extent. From an experimental point of view final states involving two leptons are preferable. In the yellow region this fraction is marked in black. In the red region $Z$ decays leptonically in $7 \%$ of the cases. In the green region, no leptonic decay is available. Here the $b \bar{b}$ final state of the Higgs can be used. This channel may even serve as a discovery channel for the Higgs boson.

Returning once more to our chosen decay chain, $\tilde{q} \rightarrow \tilde{\chi}_{2}^{0} q \rightarrow \tilde{l} l q \rightarrow \tilde{\chi}_{1}^{0} l l q$, it is clear from Figs. 2 that the initiating squark must be $\tilde{q}_{L}$ or $\tilde{b}$. Furthermore Fig. 5 shows that for low $\tan \beta$ the sleptonic decay of $\tilde{\chi}_{2}^{0}$ is open in a large fraction of the $m_{1 / 2}-m_{0}$ plane.

\subsection{Other constraints}

Much of these parameter planes considered in Figs. 15 5 are actually excluded or disfavoured by observations. The left part (small $m_{1 / 2}$ ) is typically excluded by the lower bound on the Higgs mass, and the region of 'large' $m_{0}+m_{1 / 2}$ is excluded by the WMAP data [39, 40], since a too high contribution to the Cold Dark Matter (relic LSP) density is produced. Such bounds have been explored in considerable detail for the so-called 'PostLEP' SUSY benchmark points [34, 35]. For the SPS points, see Ref. [36]. Also, there are constraints from the non-emergence of unphysical vacua during the renormalisation-group running from the high scale [37]. These typically rule out a sector at low $m_{0}$ and high $m_{1 / 2}$ that may extend beyond that excluded by charged LSP. We shall here ignore such additional constraints, since they depend somewhat on the assumptions which are adopted. In particular, tunneling into them may take longer than the age of the Universe.

\section{Summary of SPS 1a}

We have shown that the squark and gluino initiated cascade decays seen at the benchmark point and slope SPS 1a are not atypical of a large portion of the parameter space. In this section we will go on to explore SPS 1a in more detail, and introduce our second SPS 1a point on the line.

\subsection{The SPS 1a line and points}

The SPS 1a benchmark line is defined as the masses and couplings of supersymmetric particles at the $\mathrm{TeV}$ scale as evolved from the GUT scale mSUGRA inputs

$$
\begin{aligned}
& m_{0}=-A_{0}=0.4 m_{1 / 2}, \\
& \tan \beta=10, \quad \mu>0,
\end{aligned}
$$

by version 7.58 of the program ISAJET [30]. Elsewhere in this report when the mSUGRA GUT scale parameters are referred to it is to be understood that the low energy parameters are obtained in this way. 

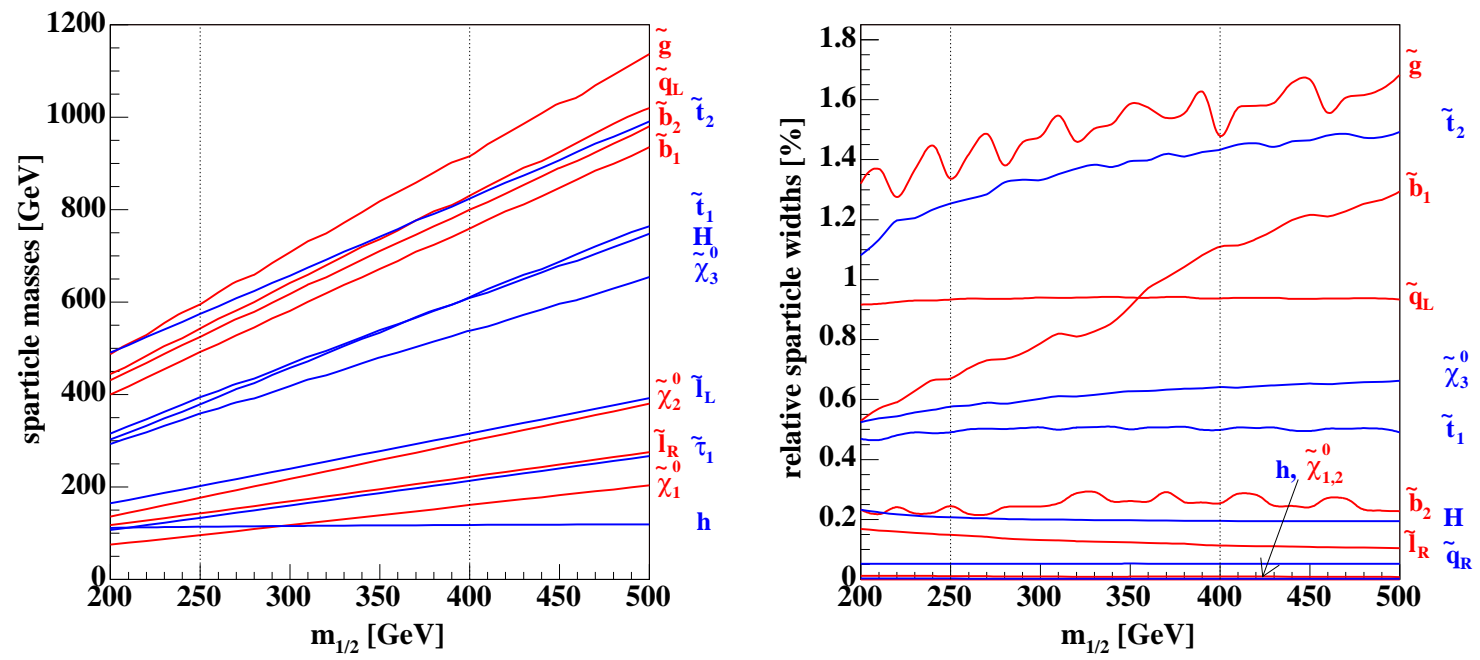

Figure 6: Masses (left) and relative widths (right) of relevant sparticles as $m_{1 / 2}, m_{0}$ and $A_{0}$ are varied along the SPS 1a slope, defined by Eq. (3.1). The vertical dotted lines represent SPS 1a points $(\alpha)$ and $(\beta)$.

In addition, we define two points $(\alpha)$ and $(\beta)$ on the SPS 1a line according to:

$$
\begin{aligned}
& (\alpha): \quad m_{0}=100 \mathrm{GeV}, \quad m_{1 / 2}=250 \mathrm{GeV}, \\
& (\beta): \quad m_{0}=160 \mathrm{GeV}, \quad m_{1 / 2}=400 \mathrm{GeV} \text {. }
\end{aligned}
$$

The first point $(\alpha)$ is the 'basic' SPS 1a point of Ref. [21] and studied in Ref. [27, 28], while the second $(\beta)$ is a new, less optimistic scenario with a reduced cross-section for the decay chain.

The masses of particles relevant for our analysis are shown in Fig. 6 (left), moving along the SPS 1a line by varying $m_{1 / 2}$. The values of the masses at points $(\alpha)$ and $(\beta)$ can be seen from the vertical dotted lines. As expected, all the masses except the lightest Higgs boson mass increase linearly with $m_{1 / 2}$. Neither the heaviest neutralino mass nor the chargino masses are shown; to a good approximation, $m_{\tilde{\chi}_{2}^{ \pm}} \simeq m_{\tilde{\chi}_{3}^{0}} \simeq m_{\tilde{\chi}_{4}^{0}}$ and $m_{\tilde{\chi}_{1}^{ \pm}} \simeq m_{\tilde{\chi}_{2}^{0}}$. Similarly, the masses of $H^{ \pm}$and $A$ are not shown, but $m_{H^{ \pm}} \simeq m_{A} \simeq m_{H}$.

For the points $(\alpha)$ and $(\beta)$, these masses are further detailed in Table 1, with the masses of the particles in our chosen decay chain displayed in bold.

The relative widths (width divided by the mass) of the decaying sparticles are shown in Fig. 6 (right), and are everywhere less than $2 \%$ of the mass. The wiggles in some of these curves (as well as in some of the branching ratio curves below) are due to limited precision in ISAJET [38]. As will be discussed, these widths contribute to a blurring of the kinematical endpoints, and will thus be reflected in the mass determination.

\subsection{Sparticle production}

The cross-sections for producing supersymmetric particles at the LHC are for moderate values of $m_{1 / 2}$ rather high. This can be seen in Fig. 7 which shows the dominating sparticle 


\begin{tabular}{|l|c|c|c|c|c|c|c|c|c|}
\hline Point & $\tilde{g}$ & $\tilde{d}_{L}$ & $\tilde{d}_{R}$ & $\tilde{u}_{L}$ & $\tilde{u}_{R}$ & $\tilde{b}_{2}$ & $\tilde{b}_{1}$ & $\tilde{t}_{2}$ & $\tilde{t}_{1}$ \\
\hline$(\alpha)$ & $\mathbf{5 9 5 . 2}$ & $\mathbf{5 4 3 . 0}$ & 520.1 & $\mathbf{5 3 7 . 2}$ & 520.5 & $\mathbf{5 2 4 . 6}$ & $\mathbf{4 9 1 . 9}$ & 574.6 & 379.1 \\
$(\beta)$ & $\mathbf{9 1 5 . 5}$ & $\mathbf{8 3 0 . 1}$ & 799.5 & $\mathbf{8 2 6 . 3}$ & 797.3 & $\mathbf{8 0 0 . 2}$ & $\mathbf{7 5 9 . 4}$ & 823.8 & 610.4 \\
\hline \hline & $\tilde{e}_{L}$ & $\tilde{e}_{R}$ & $\tilde{\tau}_{2}$ & $\tilde{\tau}_{1}$ & $\tilde{\nu}_{e_{L}}$ & $\tilde{\nu}_{\tau_{L}}$ & & $H^{ \pm}$ & $A$ \\
\hline$(\alpha)$ & 202.1 & $\mathbf{1 4 3 . 0}$ & 206.0 & 133.4 & 185.1 & 185.1 & & 401.8 & 393.6 \\
$(\beta)$ & 315.6 & $\mathbf{2 2 1 . 9}$ & 317.3 & 213.4 & 304.1 & 304.1 & & 613.9 & 608.3 \\
\hline \hline & $\tilde{\chi}_{4}^{0}$ & $\tilde{\chi}_{3}^{0}$ & $\tilde{\chi}_{2}^{0}$ & $\tilde{\chi}_{1}^{0}$ & $\tilde{\chi}_{2}^{ \pm}$ & $\tilde{\chi}_{1}^{ \pm}$ & & $H$ & $h$ \\
\hline$(\alpha)$ & 377.8 & 358.8 & $\mathbf{1 7 6 . 8}$ & $\mathbf{9 6 . 1}$ & 378.2 & 176.4 & & 394.2 & 114.0 \\
$(\beta)$ & 553.3 & 538.4 & $\mathbf{2 9 9 . 1}$ & $\mathbf{1 6 1 . 0}$ & 553.3 & 299.0 & & 608.9 & 117.9 \\
\hline
\end{tabular}

Table 1: Masses $[\mathrm{GeV}]$ for the considered SPS 1a points $(\alpha)$ and $(\beta)$ of Eq. (3.2).

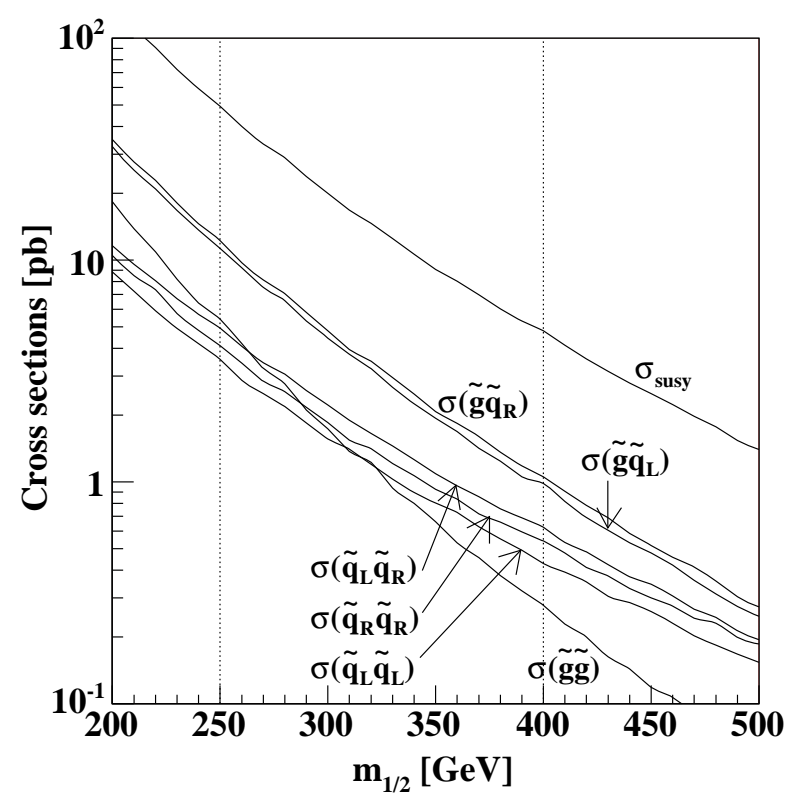

Figure 7: Cross-sections as $m_{1 / 2}, m_{0}$ and $A_{0}$ are varied along the SPS 1a slope, defined by Eq. (3.1). The vertical dotted lines represent SPS 1a points $(\alpha)$ and $(\beta)$.

pair production cross-sections, as $m_{1 / 2}$ is varied along the SPS 1a line. Notice that these cross-sections fall very rapidly as $m_{1 / 2}$ is increased, which will cause repercussions in the analysis of SPS 1a $(\beta)$.

The cross-sections for gluino-gluino, gluino-squark and squark-squark pair productions are detailed in Table 2 for the two chosen analysis points, together with the SUSY total rate. Of course since other supersymmetric particle pairs may contribute to the total SUSY rate it is not simply a sum of the other numbers in the table.

These supersymmetric particle pairs are predominantly produced by QCD interactions of quarks and gluons in the colliding protons. For gluino pairs this is mainly due to $g g \rightarrow \tilde{g} \tilde{g}$ via t-channel gluino exchange and s-channel gluons, and at a much smaller rate $q \bar{q} \rightarrow \tilde{g} \tilde{g}$ via s-channel gluons. Squark pairs with the same handedness have the dominant production 
process $q q \rightarrow \tilde{q}_{L / R} \tilde{q}_{L / R}$ with a t-channel gluino exchange, but can also be produced via $g g \rightarrow \tilde{q}_{L / R} \tilde{q}_{L / R}$ with an s-channel gluon, or via $q \bar{q} \rightarrow \tilde{q}_{L / R} \tilde{q}_{L / R}$ with both t-channel gluino and s-channel gluons. For opposite handedness, $\tilde{q}_{L} \tilde{q}_{R}$ production, the s-channel gluon exchange processes [and thus $g g \rightarrow \tilde{q}_{L} \tilde{q}_{R}$ at tree-level] are disallowed. Lastly, the $\tilde{g} \tilde{q}$ final states are produced by the process $g q \rightarrow \tilde{g} \tilde{q}$ mediated by t-channel squark or gluino exchanges and s-channel quarks.

\begin{tabular}{|r|r|r|r|r|r|r|r|}
\hline & $\sigma(\mathrm{SUSY})$ & $\sigma(\tilde{g} \tilde{g})$ & $\sigma\left(\tilde{g} \tilde{q}_{L}\right)$ & $\sigma\left(\tilde{g} \tilde{q}_{R}\right)$ & $\sigma\left(\tilde{q}_{L} \tilde{q}_{L}\right)$ & $\sigma\left(\tilde{q}_{L} \tilde{q}_{R}\right)$ & $\sigma\left(\tilde{q}_{R} \tilde{q}_{R}\right)$ \\
\hline$(\alpha)$ & 49.3 & 5.3 & 11.4 & 12.3 & 3.5 & 4.8 & 4.1 \\
$(\beta)$ & 4.76 & 0.29 & 0.97 & 1.06 & 0.44 & 0.61 & 0.53 \\
\hline
\end{tabular}

Table 2: Selected supersymmetry cross-sections in pb.

However, these particle pair cross-sections are not the production cross-sections that are relevant to our analysis of the decay chain, since it does not matter from where the parent squark originates. Therefore we should also be counting, for example, gluinos which decay into squarks as possible sources of the decay chain. In Table 3 we divide the sparticle productions rates into 'direct' and 'indirect' contributions, reflecting production rates from sparticles in the 'initial' supersymmetric state as opposed to their generation from the decay of a parent sparticle. Furthermore, if the 'initial' supersymmetric state contains two possible parents then the chance of generating the desired decay chain is doubled ${ }^{2}$. Therefore the pair-production rates with two possible parent particles are counted twice. Again, one cannot simply add the various contributions from Table 2 to obtain the 'direct' rates of Table 3, since they include contributions from squarks or gluinos produced in association with other supersymmetric particles.

\begin{tabular}{|ll|r|r|r|r|r|r|r|}
\hline & & $\Sigma(\tilde{g})$ & $\Sigma\left(\tilde{q}_{L}\right)$ & $\Sigma\left(\tilde{q}_{R}\right)$ & $\Sigma\left(\tilde{b}_{1}\right)$ & $\Sigma\left(\tilde{b}_{2}\right)$ & $\Sigma\left(\tilde{t}_{1}\right)$ & $\Sigma\left(\tilde{\chi}_{2}^{0}\right)$ \\
\hline$(\alpha)$ & Direct & 35.4 & 24.6 & 25.8 & 1.4 & 0.9 & 3.4 & 1.8 \\
& Indirect & - & 8.2 & 14.6 & 6.3 & 3.5 & 5.6 & 16.0 \\
& Total & 35.4 & 32.8 & 40.4 & 7.7 & 4.3 & 9.0 & 17.8 \\
\hline \multirow{2}{*}{$\beta)$ Direct } & 2.71 & 2.64 & 2.80 & 0.10 & 0.06 & 0.23 & 0.23 \\
& Indirect & - & 0.58 & 1.00 & 0.40 & 0.25 & 0.64 & 1.44 \\
& Total & 2.71 & 3.21 & 3.79 & 0.50 & 0.31 & 0.87 & 1.67 \\
\hline
\end{tabular}

Table 3: Selected sparticle production rates in pb.

For low $m_{1 / 2}$ values $\tilde{\chi}_{2}^{0}$ will decay dominantly to a stau and a tau, see Fig. 8. Here, the decay mode of interest is to a right-handed slepton, $\tilde{l}_{R}$, and a lepton, and is at the level of $10 \%$. For higher values of $m_{1 / 2}$, the mass difference between $\tilde{\chi}_{1}^{0}$ and $\tilde{\chi}_{2}^{0}$ grows sufficiently to allow the decay $\tilde{\chi}_{2}^{0} \rightarrow \tilde{\chi}_{1}^{0} h$. At $(\beta)$, even though the Higgs channel takes a significant $30 \%$ of the decay width, it was not used in this study. Due to the accuracy of lepton reconstruction compared to jet reconstruction, only in the case of very low lepton channel statistics can the Higgs channel improve on the results obtained with the slepton channel.

\footnotetext{
${ }^{2}$ The total branching ratio for the decay is sufficiently small that the chance of generating two of the desired decay chains is tiny.
} 


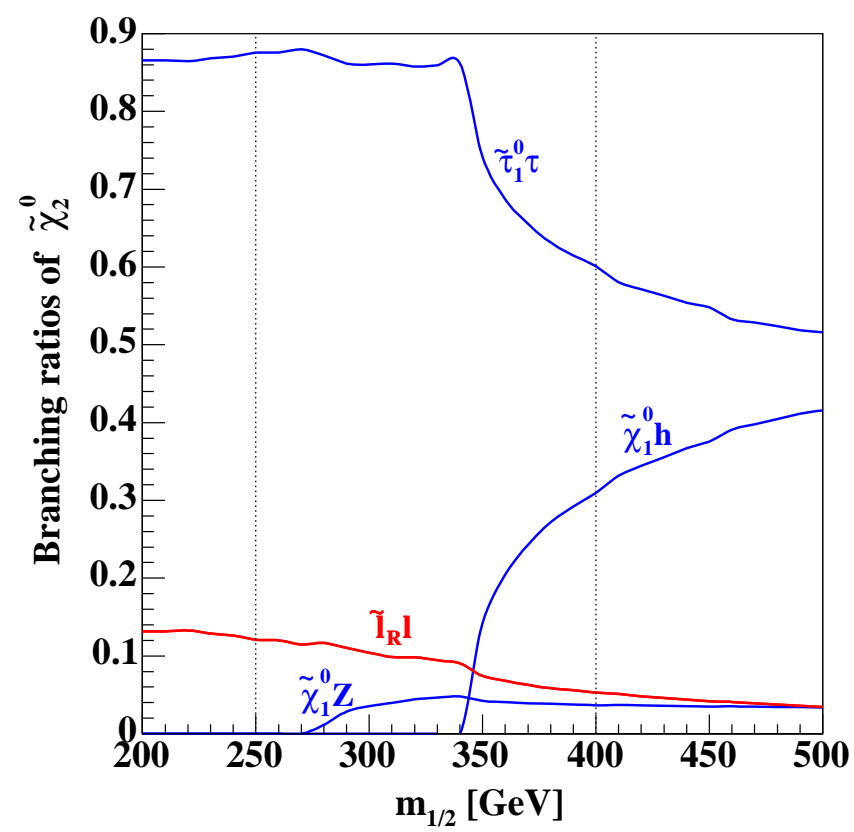

Figure 8: Branching ratios of $\tilde{\chi}_{2}^{0}$ as $m_{1 / 2}, m_{0}$ and $A_{0}$ are varied along the SPS 1a slope. The vertical dotted lines represent SPS 1a points $(\alpha)$ and $(\beta)$.

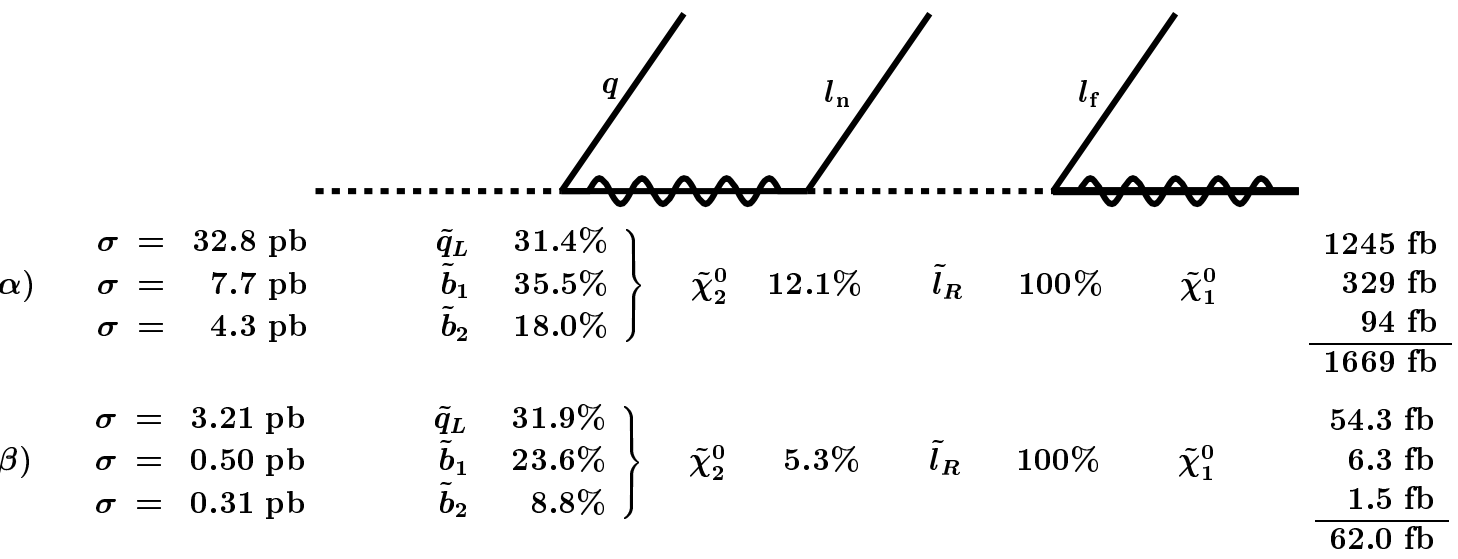

Figure 9: The SPS 1a cascade decay chain.

\subsection{The cascade}

The cross-sections and branching ratios of our chosen decay chains

$$
\begin{aligned}
& \tilde{q}_{L} \rightarrow \tilde{\chi}_{2}^{0} q \rightarrow \tilde{l}_{R}^{\mp} l_{\mathrm{n}}^{ \pm} q \rightarrow \tilde{\chi}_{1}^{0} l_{\mathrm{f}}^{\mp} l_{\mathrm{n}}^{ \pm} q \\
& \tilde{b}_{1} \rightarrow \tilde{\chi}_{2}^{0} b \rightarrow \tilde{l}_{R}^{\mp} l_{\mathrm{n}}^{ \pm} b \rightarrow \tilde{\chi}_{1}^{0} l_{\mathrm{f}}^{\mp} l_{\mathrm{n}}^{ \pm} b \\
& \tilde{b}_{2} \rightarrow \tilde{\chi}_{2}^{0} b \rightarrow \tilde{l}_{R}^{\mp} l_{\mathrm{n}}^{ \pm} b \rightarrow \tilde{\chi}_{1}^{0} l_{\mathrm{f}}^{\mp} l_{\mathrm{n}}^{ \pm} b
\end{aligned}
$$

are summarized in Fig. 9 for the two SPS 1a points. Since the left-handed up and down squarks, $\tilde{u}_{L}$ and $\tilde{d}_{L}$, have very similar masses (at $537.2 \mathrm{GeV}$ and $543.0 \mathrm{GeV}$ respectively), for $(\alpha)$, they are in the above jointly referred to as $\tilde{q}_{L}$, and for this analysis will be grouped 
together. For the fraction of decay chains which commence from a sbottom, $\tilde{b}_{1}$ is responsible for $78 \%$ or so, leaving us rather insensitive to the contribution from $\tilde{b}_{2}$.

\section{Mass distributions}

The longer a decay chain is, the more information it contains. To extract the masses of the supersymmetric particles in the decay we require at least as many kinematic endpoint measurements as unknown masses. In the lower part of the decay chain, where the secondlightest neutralino decays via $\tilde{\chi}_{2}^{0} \rightarrow \tilde{l}_{R} l \rightarrow \tilde{\chi}_{1}^{0} l l$, there are three unknown masses: $m_{\tilde{\chi}_{2}^{0}}, m_{\tilde{l}_{R}}$ and $m_{\tilde{\chi}_{1}^{0}}$. However, only two particle momenta are measured, those of the two leptons, from which only one mass distribution can be constructed, $m_{l l}$. The system is highly underdetermined; one cannot extract the three masses, only a relation between them.

When a squark is added to the head of the decay chain, $\tilde{q} \rightarrow \tilde{\chi}_{2}^{0} q \rightarrow \tilde{l}_{R} l_{\mathrm{n}} q \rightarrow \tilde{\chi}_{1}^{0} l_{\mathrm{f}} l_{\mathrm{n}} q$, three particles can be collected, and one can construct four mass distributions, $m_{l l}, m_{q l_{\mathrm{n}}}$, $m_{q l_{\mathrm{f}}}$ and $m_{q l l}$, where following the notation of Refs. [31, 32], we denote the first emitted lepton $l_{\mathrm{n}}$ ('n' for 'near') and the second $l_{\mathrm{f}}$ ('f' for 'far'). In principle this is just sufficient for extracting the four unknown masses: $m_{\tilde{q}}, m_{\tilde{\chi}_{2}^{0}}, m_{\tilde{l}_{R}}$ and $m_{\tilde{\chi}_{1}^{0}}$. However, in order to use the distributions $m_{q l_{\mathrm{n}}}$ and $m_{q l_{\mathrm{f}}}$, we need to be able to distinguish $l_{\mathrm{n}}$ from $l_{\mathrm{f}}$. Since this is

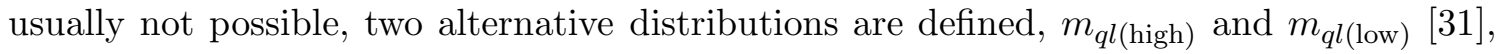
constructed by selecting for each event the largest and smallest values of $m_{q l}$ respectively.

As will be detailed later in this section, the expressions for these kinematic endpoints are not always linearly independent, so these four endpoints are not always sufficient to determine the masses in the decay chain. In this circumstance one must look for other endpoint measurements.

Correlations between different mass distributions can provide further measurements.

For example, one may define the mass distribution $m_{q l l\left(\theta>\frac{\pi}{2}\right)}$ identically to the $m_{q l l}$ distribution but with the additional constraint

$$
m_{l l}^{\max } / \sqrt{2}<m_{l l}<m_{l l}^{\max } .
$$

This cut on $m_{l l}$ translates directly into a cut on the angle $\theta$ between the two leptons in the rest frame of $\tilde{l}_{R}$ [1]. In terms of this angle, $m_{l l}$ is given by

$$
m_{l l}=m_{l l}^{\max } \sqrt{(1-\cos \theta) / 2}
$$

so a constraint of the form (4.1) directly corresponds to $\theta>\frac{\pi}{2}$. The simplicity of this constraint allows one to find an analytic expression for the minimum of the $m_{q l l\left(\theta>\frac{\pi}{2}\right)}$ distribution.

In principle, other correlations between mass distributions could be used, but they are limited by the lack of analytic expressions for the associated extrema. It is no doubt possible to construct simple constraints for which analytic expressions for minima or maxima of mass distributions are possible, but this will not be investigated further in this study.

If we were to also include a parent gluino at the head of the decay chain, $\tilde{g} \rightarrow \tilde{q} q \rightarrow$ $\tilde{\chi}_{2}^{0} q q \rightarrow \tilde{l}_{R} l q q \rightarrow \tilde{\chi}_{1}^{0} l l q q$, we would have an extra quark momentum at our disposal and could construct with it seven more ('primary') mass distributions. 
$\alpha$
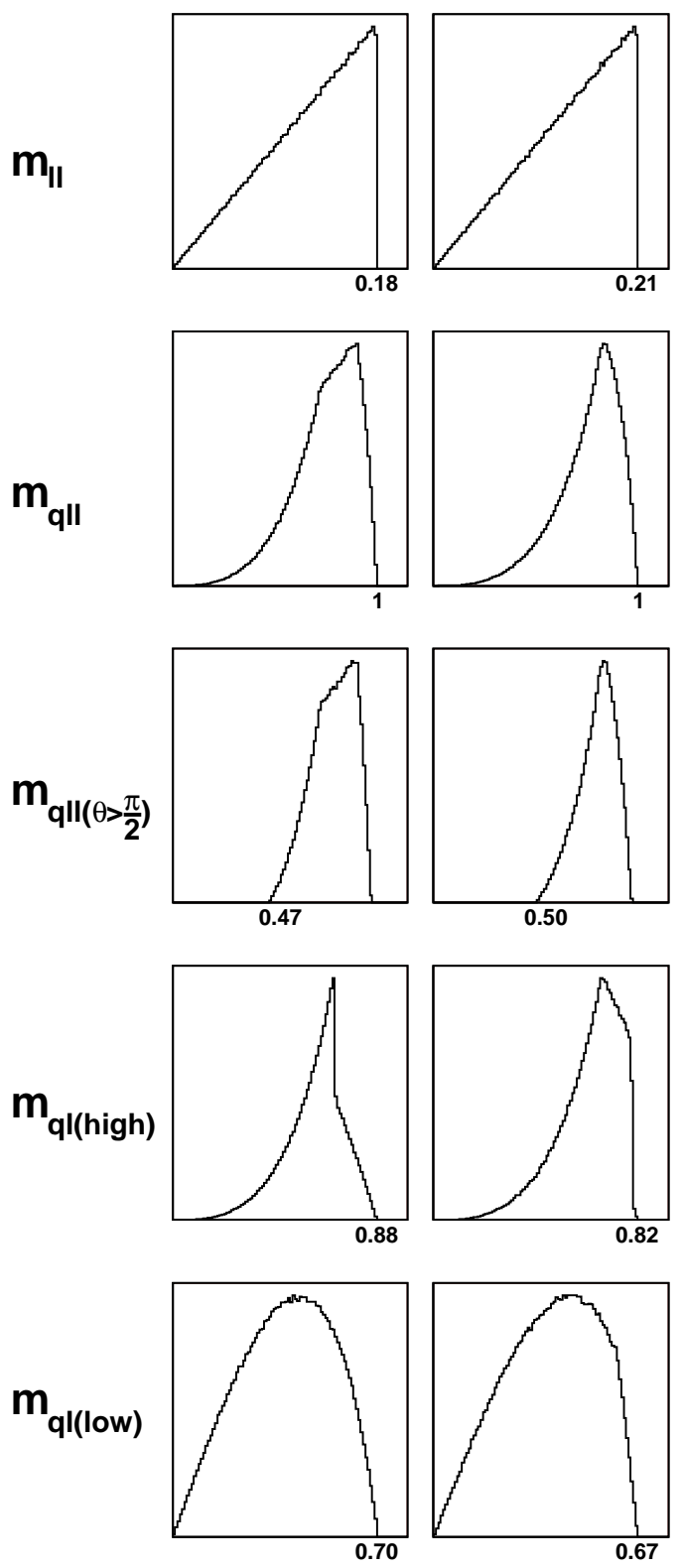

(i)
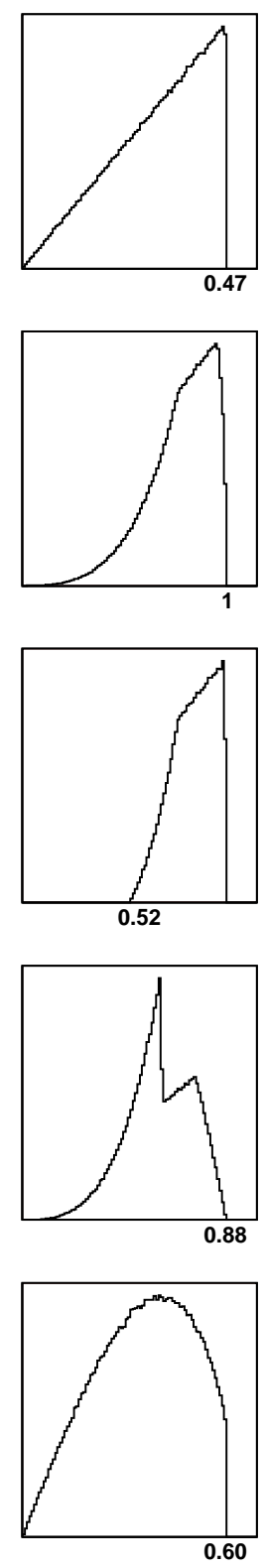

(ii)
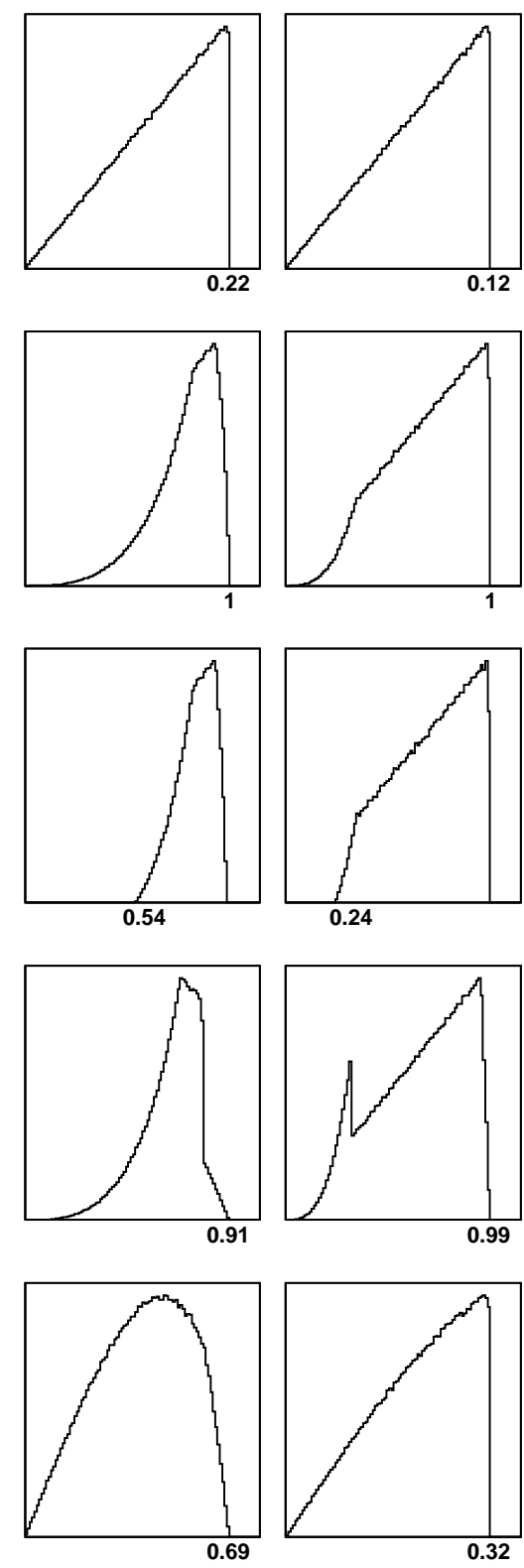

Figure 10: Theoretical mass distributions for SPS 1a $(\alpha)$ and $(\beta)$, as well as for three other mass scenarios, denoted (i), (ii) and (iii). Kinematic endpoints are given in units of $m_{q l l}^{\max }$. (More details will be given in 42 .)

\subsection{Theory curves of invariant mass distributions}

In Fig. 10 we show 'theory' versions of the five mass distributions discussed above for SPS 1a $(\alpha)$ and $(\beta)$, and three other mass scenarios. These distributions reflect the parton level only, where the quark and leptons are assumed to be perfectly reconstructed, and particle widths have been neglected, suppressing a mild smearing of the distributions. Leptons and 
quarks are assumed massless; at worst, i.e. for an endpoint involving a $4.8 \mathrm{GeV}$ b-quark, this approximation gives a value for $m_{\text {qll }}^{\max }$ which is wrong by $5.7 \mathrm{MeV}$ at SPS 1a $(\alpha)$, which is negligible. Furthermore a common squark mass is used; in reality, the experimental distributions would be a sum of several similar distributions shifted typically by a few percent, depending on the differences between the squark masses.

These theory curves have been generated from the phase space only, with no matrix elements inserted. For the two-body decay of scalar particles this is not a problem; the daughter particles are always emitted back-to-back in the rest frame of the parent and since a scalar particle provides no intrinsic direction the decay will be isotropic. The $m_{l l}$ distribution, for example, is very well described by phase-space considerations alone since the scalar $\tilde{l}_{R}$ removes any spin-correlations between the two leptons. For the decays of fermions this is not so clear; the parent's spin picks out a particular direction so the decay need not be isotropic. One would expect that spin-correlations must be fully taken into account by calculating the full matrix elements for the decay chain. In practice, while these spin-correlations are indeed very significant in individual processes, when one sums up final states containing leptons with positive and negative charge (which are produced in equal proportion) these spin correlations cancel out [43]. We have confirmed this assertion analytically.

Most of the distributions of Fig. 10 show a strong dependence on the scenario. Only the $m_{l l}$ distribution is independent of the masses involved, which is easily understood from (4.2) together with the isotropic decay of $\tilde{l}_{R}$. The shape is easy to fit, and since only leptons are involved, the experimental resolution is high. The presence of the quark reduces the precision with which the other distributions may be measured. For $m_{q l l}$ there is a clear dependence on the masses involved, the main feature being the length of the straight section, ranging from infinitesimal to the entire distribution. However, the maximum edge itself is quite well described by a straight line, at least for the latter part. Next, the constrained $m_{q l l}$ distribution differs from $m_{q l l}$ for smaller invariant masses. Its rise from threshold is not very well described by a straight line making the measurement of the minimum value rather imprecise. Both $m_{q l}$ distributions are 'composite' distributions, based on entries from $m_{q l_{\mathrm{n}}}$ and $m_{q l_{\mathrm{f}}}$. This double nature is readily apparent for $m_{q l \text { (high), }}$, which has a wide variety of shapes. Particularly dangerous are mass scenarios like $(\beta)$ or (ii), where the 'foot' which forms the last part of the edge can be hidden by backgrounds, giving a false maximum. In Sections 5 - 6 these considerations will come up in relation to $(\beta)$. For $m_{q l \text { (low) }}$ a similar danger arises in cases like (i) where what appears to be an approximately linear descent to zero suddenly turns into a vertical drop towards the end.

These theory curves must serve as guidance for our determination of the endpoints. As a first stage they should be the basis for the choice of functions with which the edges of the different distributions are fitted. As a second stage the more ambitious goal may be to fit not only the endpoint, but the entire distribution.

\subsection{Formulae for kinematic endpoints}

The invariant masses of various subsets of particles can be determined from kinematical endpoints and thresholds, as discussed in [31]. We confirm the results relevant for our 
analysis:

$$
\begin{aligned}
& \left(m_{l l}^{\max }\right)^{2}=\left(m_{\tilde{\chi}_{2}^{0}}^{2}-m_{\tilde{l}_{R}}^{2}\right)\left(m_{\tilde{l}_{R}}^{2}-m_{\tilde{\chi}_{1}^{0}}^{2}\right) / m_{\tilde{l}_{R}}^{2} \\
& \left(m_{q l l}^{\max }\right)^{2}=\left\{\begin{array}{lll}
\frac{\left(m_{\tilde{q}_{L}}^{2}-m_{\tilde{\chi}_{2}^{0}}^{2}\right)\left(m_{\tilde{\chi}_{2}^{0}}^{2}-m_{\tilde{\chi}_{1}^{0}}^{2}\right)}{m_{\tilde{\chi}_{2}^{0}}^{2}} & \text { for } & \frac{m_{\tilde{q}_{L}}}{m_{\tilde{\chi}_{2}^{0}}}>\frac{m_{\tilde{\chi}_{2}^{0}}}{m_{\tilde{l}_{R}}} \frac{m_{\tilde{l}_{R}}}{m_{\tilde{\chi}_{1}^{0}}} \\
\frac{\left(m_{\tilde{q}_{L}}^{2} m_{\tilde{l}_{R}}^{2}-m_{\tilde{\chi}_{2}^{0}}^{2} m_{\tilde{\chi}_{1}^{0}}^{2}\right)\left(m_{\tilde{\chi}_{2}^{0}}^{2}-m_{\tilde{l}_{R}}^{2}\right)}{m_{\tilde{\chi}_{2}^{0}}^{2} m_{\tilde{l}_{R}}^{2}} & \text { for } & \frac{m_{\tilde{\chi}_{2}^{0}}}{m_{\tilde{l}_{R}}}>\frac{m_{\tilde{l}_{R}}}{m_{\tilde{\chi}_{1}^{0}}} \frac{m_{\tilde{q}_{L}}}{m_{\tilde{\chi}_{2}^{0}}} \\
\frac{\left(m_{\tilde{q}_{L}}^{2}-m_{\tilde{l}_{R}}^{2}\right)\left(m_{\tilde{l}_{R}}^{2}-m_{\tilde{\chi}_{1}^{0}}^{2}\right)}{m_{\tilde{l}_{R}}^{2}} & \text { for } & \frac{m_{\tilde{l}_{R}}}{m_{\tilde{\chi}_{1}^{0}}}>\frac{m_{\tilde{q}_{L}}}{m_{\tilde{\chi}_{2}^{0}}} \frac{m_{\tilde{\chi}_{2}^{0}}}{m_{\tilde{l}_{R}}} \\
\left(m_{\tilde{q}_{L}}-m_{\tilde{\chi}_{1}^{0}}\right)^{2} & \text { otherwise }
\end{array}\right. \\
& \left(m_{q l(\mathrm{low})}^{\max }, m_{q l(\mathrm{high})}^{\max }\right)=\left\{\begin{array}{lll}
\left(m_{q l_{\mathrm{n}}}^{\max }, m_{q l_{\mathrm{f}}}^{\max }\right) & \text { for } & 2 m_{\tilde{l}_{R}}^{2}>m_{\tilde{\chi}_{1}^{0}}^{2}+m_{\tilde{\chi}_{2}^{0}}^{2}>2 m_{\tilde{\chi}_{1}^{0}} m_{\tilde{\chi}_{2}^{0}} \\
\left(m_{q l(\mathrm{eq})}^{\max }, m_{q l_{\mathrm{f}}}^{\max }\right) & \text { for } & m_{\tilde{\chi}_{1}^{0}}^{2}+m_{\tilde{\chi}_{2}^{0}}^{2}>2 m_{\tilde{l}_{R}}^{2}>2 m_{\tilde{\chi}_{1}^{0}} m_{\tilde{\chi}_{2}^{0}} \\
\left(m_{q l(\mathrm{eq})}^{\max }, m_{q l_{\mathrm{n}}}^{\max }\right) & \text { for } & m_{\tilde{\chi}_{1}^{0}}^{2}+m_{\tilde{\chi}_{2}^{0}}^{2}>2 m_{\tilde{\chi}_{1}^{0}} m_{\tilde{\chi}_{2}^{0}}>2 m_{\tilde{l}_{R}}^{2}
\end{array}\right. \\
& \left(m_{q l_{\mathrm{n}}}^{\max }\right)^{2}=\left(m_{\tilde{q}_{L}}^{2}-m_{\tilde{\chi}_{2}^{0}}^{2}\right)\left(m_{\tilde{\chi}_{2}^{0}}^{2}-m_{\tilde{l}_{R}}^{2}\right) / m_{\tilde{\chi}_{2}^{0}}^{2} \\
& \left(m_{q l_{\mathrm{f}}}^{\max }\right)^{2}=\left(m_{\tilde{q}_{L}}^{2}-m_{\tilde{\chi}_{2}^{0}}^{2}\right)\left(m_{\tilde{l}_{R}}^{2}-m_{\tilde{\chi}_{1}^{0}}^{2}\right) / m_{\tilde{l}_{R}}^{2} \\
& \left(m_{q l(\mathrm{eq})}^{\max }\right)^{2}=\left(m_{\tilde{q}_{L}}^{2}-m_{\tilde{\chi}_{2}^{0}}^{2}\right)\left(m_{\tilde{l}_{R}}^{2}-m_{\tilde{\chi}_{1}^{0}}^{2}\right) /\left(2 m_{\tilde{l}_{R}}^{2}-m_{\tilde{\chi}_{1}^{0}}^{2}\right) \\
& \left(m_{q l l\left(\theta>\frac{\pi}{2}\right)}^{\min }\right)^{2}=\left[\left(m_{\tilde{q}_{L}}^{2}+m_{\tilde{\chi}_{2}^{0}}^{2}\right)\left(m_{\tilde{\chi}_{2}^{0}}^{2}-m_{\tilde{l}_{R}}^{2}\right)\left(m_{\tilde{l}_{R}}^{2}-m_{\tilde{\chi}_{1}^{0}}^{2}\right)\right. \\
& -\left(m_{\tilde{q}_{L}}^{2}-m_{\tilde{\chi}_{2}^{0}}^{2}\right) \sqrt{\left(m_{\tilde{\chi}_{2}^{0}}^{2}+m_{\tilde{l}_{R}}^{2}\right)^{2}\left(m_{\tilde{l}_{R}}^{2}+m_{\tilde{\chi}_{1}^{0}}^{2}\right)^{2}-16 m_{\tilde{\chi}_{2}^{0}}^{2} m_{\tilde{l}_{R}}^{4} m_{\tilde{\chi}_{1}^{0}}^{2}} \\
& \left.+2 m_{\tilde{l}_{R}}^{2}\left(m_{\tilde{q}_{L}}^{2}-m_{\tilde{\chi}_{2}^{0}}^{2}\right)\left(m_{\tilde{\chi}_{2}^{0}}^{2}-m_{\tilde{\chi}_{1}^{0}}^{2}\right)\right] /\left(4 m_{\tilde{l}_{R}}^{2} m_{\tilde{\chi}_{2}^{0}}^{2}\right)
\end{aligned}
$$

where 'low' and 'high' on the left-hand side in Eq. (4.5) refer to minimising and maximising with respect to the choice of lepton. Furthermore 'min' in Eq. (4.9) refers to the threshold in the subset of the $m_{q l l}$ distribution for which the angle between the two lepton momenta (in the slepton rest frame) exceeds $\pi / 2$, corresponding to the mass range (4.1).

Notice that the different cases listed in Eq. (4.4) are distinguished by mass ratios of neighbouring particles in the hierarchy, $m_{\tilde{q}_{L}} / m_{\tilde{\chi}_{2}^{0}}, m_{\tilde{\chi}_{2}^{0}} / m_{\tilde{l}_{R}}$ and $m_{\tilde{l}_{R}} / m_{\tilde{\chi}_{1}^{0}}$. Since each decay in the chain involves two massive particles and one massless one, the boosts from one rest frame to another are conveniently expressed in terms of such mass ratios. 


\subsection{Inversion formulae}

Once the endpoints of the distributions have been measured, the masses may be extracted. In principle, this can be done in two ways. If the number of endpoint measurements coincides with the number of unknown masses, one may analytically invert the expressions for the endpoints to give explicit formulae for the masses in terms of the endpoints. If more endpoints are known, the system becomes overconstrained and the measurements must be weighted according to their uncertainties. The analytic method cannot easily handle such a situation. Instead the more flexible approach of numerical fit must be used.

However, the analytic inversion is more transparent than the numerical treatment, and reveals some interesting features of how the masses are related to the endpoints. It is also practical to use the analytical method in combination with the numerical method to provide initial values for the fits. Consequently we will discuss the inversion of the endpoint formulae in some detail in this subsection.

The four principal endpoints $m_{l l}^{\max }, m_{q l l}^{\max }, m_{q l(\mathrm{low})}^{\max }$ and $m_{q l(\mathrm{high})}^{\max }$ are given by Eqs. (4.3)(4.5). While $m_{l l}^{\max }$ is given by one unique expression, the others have different representations for different mass spectra. To perform the inversion, each combination of the endpoint expressions must be considered separately. There are four representations of $m_{\text {qll }}^{\max }$ and three representations of $\left(m_{q l(\text { low })}^{\max }, m_{q l(\text { high })}^{\max }\right)$. Each overall combination corresponds to a unique region in mass space $\left(m_{\tilde{q}_{L}}, m_{\tilde{\chi}_{2}^{0}}, m_{\tilde{l}_{R}}, m_{\tilde{\chi}_{1}^{0}}\right)$ and can be labeled by $(i, j)$, where $i$ and $j$ denote the region of applicability of the $m_{q l l}$ and $m_{q l}$ endpoints, respectively, as given in Eqs. (4.4)-(4.5).

However, not all of the $4 \times 3$ combinations are physical. In particular the regions (2,1), $(2,2)$ and $(3,3)$ are not possible. For $(2,1)$ this can be seen by simultaneously trying to satisfy the mass constraints (2) of Eq. (4.4) and (1) of Eq. (4.5).

Additionally, for the regions (2,3), (3,1) and (3,2) the endpoint expressions are not linearly independent. In each of these three regions, one of the mass ratios is dominant, and a lepton (rather than a quark) is emitted from the vertex involving the dominant mass ratio. The maximum of $m_{q l l}$ occurs when this lepton travels in one direction and the other lepton together with the quark travels in the opposite direction, all observed from the rest frame of any one of the sparticles in the decay. For massless quarks and leptons one can always write

$$
m_{q l l}^{2}=2 p_{q} p_{l_{\mathrm{n}}}+2 p_{q} p_{l_{\mathrm{f}}}+2 p_{l_{\mathrm{n}}} p_{l_{\mathrm{f}}}=m_{q l(\text { high })}^{2}+m_{q l(\mathrm{low})}^{2}+m_{l l}^{2}
$$

In the three regions mentioned above, for maximum values this equation reduces to

$$
\left(m_{q l l}^{\max }\right)^{2}=\left(m_{q l(\text { high })}^{\max }\right)^{2}+\left(m_{l l}^{\max }\right)^{2}
$$

Since one of the endpoints can be expressed in terms of two others, the four endpoint measurements only transform into three independent conditions. Hence, in order to determine the masses an additional endpoint would be required for these three regions. The endpoint of $m_{q l l\left(\theta>\frac{\pi}{2}\right)}$ is therefore particularly important in such cases. However, the complicated nature of Eq. (4.9) leads to exceptionally cumbersome inversion formulae and will not be studied here. 
For the remaining six regions the inversion of the four endpoint expressions Eqs. (4.3)(4.5) is possible. Coincidentally these are actually the regions most likely to be realised in SUSY scenarios with sparticle mass unifications at the GUT scale. Inversion formulae for these regions are detailed below. For simplicity of notation we will write:

$$
a=m_{l l}^{\max }, b=m_{q l l}^{\max }, c=m_{q l(\mathrm{low})}^{\max }, d=m_{q l(\text { high })}^{\max }
$$

Region (1,1):

$$
\begin{aligned}
& m_{\tilde{\chi}_{1}^{0}}^{2}=\frac{\left(b^{2}-d^{2}\right)\left(b^{2}-c^{2}\right)}{\left(c^{2}+d^{2}-b^{2}\right)^{2}} a^{2} \\
& m_{\tilde{l}_{R}}^{2}=\frac{c^{2}\left(b^{2}-c^{2}\right)}{\left(c^{2}+d^{2}-b^{2}\right)^{2}} a^{2} \\
& m_{\tilde{\chi}_{2}^{0}}^{2}=\frac{c^{2} d^{2}}{\left(c^{2}+d^{2}-b^{2}\right)^{2}} a^{2} \\
& m_{\tilde{q}_{L}}^{2}=\frac{c^{2} d^{2}}{\left(c^{2}+d^{2}-b^{2}\right)^{2}}\left(c^{2}+d^{2}-b^{2}+a^{2}\right)
\end{aligned}
$$

Region (1,2):

$$
\begin{aligned}
& m_{\tilde{\chi}_{1}^{0}}^{2}=\frac{\left(b^{2} c^{2}-b^{2} d^{2}+c^{2} d^{2}\right)\left(2 c^{2}-d^{2}\right)}{\left(d^{2}-c^{2}\right)^{2}\left(b^{2}-d^{2}\right)} a^{2} \\
& m_{\tilde{l}_{R}}^{2}=\frac{\left(b^{2} c^{2}-b^{2} d^{2}+c^{2} d^{2}\right) c^{2}}{\left(d^{2}-c^{2}\right)^{2}\left(b^{2}-d^{2}\right)} a^{2} \\
& m_{\tilde{\chi}_{2}^{0}}^{2}=\frac{\left(2 c^{2}-d^{2}\right) c^{2} d^{2}}{\left(d^{2}-c^{2}\right)^{2}\left(b^{2}-d^{2}\right)} a^{2} \\
& m_{\tilde{q}_{L}}^{2}=\frac{c^{2} d^{2}}{\left(d^{2}-c^{2}\right)^{2}\left(b^{2}-d^{2}\right)}\left[a^{2}\left(2 c^{2}-d^{2}\right)+\left(d^{2}-c^{2}\right)\left(b^{2}-d^{2}\right)\right]
\end{aligned}
$$

Region (1,3):

$$
\begin{aligned}
& m_{\tilde{\chi}_{1}^{0}}^{2}=\frac{\left(d^{2}-c^{2}\right)\left(b^{2}-d^{2}\right)\left(b^{2}-2 c^{2}\right)}{\left(c^{2}+d^{2}-b^{2}\right)^{2} d^{2}} a^{2} \\
& m_{\tilde{l}_{R}}^{2}=\frac{\left(d^{2}-c^{2}\right)^{2}\left(b^{2}-d^{2}\right)}{\left(c^{2}+d^{2}-b^{2}\right)^{2} d^{2}} a^{2} \\
& m_{\tilde{\chi}_{2}^{0}}^{2}=\frac{\left(2 d^{2}-b^{2}\right)\left(d^{2}-c^{2}\right) c^{2}}{\left(c^{2}+d^{2}-b^{2}\right)^{2} d^{2}} a^{2} \\
& m_{\tilde{q}_{L}}^{2}=\frac{\left(2 d^{2}-b^{2}\right) c^{2}}{\left(c^{2}+d^{2}-b^{2}\right)^{2} d^{2}}\left[a^{2}\left(d^{2}-c^{2}\right)+d^{2}\left(c^{2}+d^{2}-b^{2}\right)\right]
\end{aligned}
$$

Region (4,1):

$$
\begin{aligned}
& m_{\tilde{\chi}_{1}^{0}}^{2}=m_{\tilde{\chi}_{2}^{0}}^{2}-(d a / c+a c / d) m_{\tilde{\chi}_{2}^{0}}+a^{2} \\
& m_{\tilde{l}_{R}}^{2}=m_{\tilde{\chi}_{2}^{0}}^{2}-(a c / d) m_{\tilde{\chi}_{2}^{0}} \\
& m_{\tilde{\chi}_{2}^{0}}=\frac{\left[\left(a^{2}+b^{2}\right) c^{2} d^{2}-\left(b^{2}-a^{2}\right) a^{2}\left(c^{2}+d^{2}\right)+2 a b c d \sqrt{\left(a^{2}+c^{2}-b^{2}\right)\left(a^{2}+d^{2}-b^{2}\right)}\right]}{\left[\left(a^{2} c^{2}+a^{2} d^{2}+c^{2} d^{2}\right)^{2}-4 a^{2} b^{2} c^{2} d^{2}\right] /(a c d)}
\end{aligned}
$$


$m_{\tilde{q}_{L}}=m_{\tilde{\chi}_{1}^{0}}+b$

Region (4,2):

$$
\begin{aligned}
& m_{\tilde{\chi}_{1}^{0}}=\frac{2 c^{2}-d^{2}}{d^{2}-c^{2}}\left(b-c \sqrt{\frac{b^{2}-a^{2}-d^{2}}{2 c^{2}-d^{2}}}\right) \\
& m_{\tilde{l}_{R}}^{2}=\frac{c^{2}}{2 c^{2}-d^{2}} m_{\tilde{\chi}_{1}^{0}}^{2} \\
& m_{\tilde{\chi}_{2}^{0}}^{2}=\frac{c^{2}}{2 c^{2}-d^{2}} m_{\tilde{\chi}_{1}^{0}}^{2}+\frac{a^{2} c^{2}}{d^{2}-c^{2}} \\
& m_{\tilde{q}_{L}}=m_{\tilde{\chi}_{1}^{0}}+b
\end{aligned}
$$

Region (4,3):

$$
\begin{aligned}
m_{\tilde{\chi}_{1}^{0}}^{2}= & m_{\tilde{l}_{R}}^{2}+a\left[a\left(d^{2}-c^{2}\right)-\sqrt{a^{2}\left(d^{2}-c^{2}\right)^{2}+4 c^{2} d^{2} m_{\tilde{l}_{R}}^{2}}\right] /\left(2 d^{2}\right) \\
m_{\tilde{l}_{R}}^{2}= & a^{2}\left\{-\left(d^{4}-c^{4}\right)^{2} a^{8}+\left(d^{2}-c^{2}\right)\left[c^{6}\left(2 d^{2}-3 b^{2}\right)+7 c^{4} b^{2} d^{2}+d^{4}\left(c^{2}\left(3 b^{2}-2 d^{2}\right)+b^{2} d^{2}\right)\right] a^{6}\right. \\
& +c^{2} d^{2}\left[\left(5 c^{4}-6 c^{2} d^{2}-3 d^{4}\right) b^{4}+\left(7 c^{2} d^{4}+5 c^{6}-5 c^{4} d^{2}+d^{6}\right) b^{2}+4 d^{4} c^{4}\right] a^{4} \\
& +d^{4} c^{4}\left[4 d^{2} c^{2} b^{2}-10 c^{2} b^{4}+2 c^{2} d^{4}+2 c^{4} d^{2}+c^{4} b^{2}+4 b^{6}-2 d^{2} b^{4}-d^{4} b^{2}\right] a^{2} \\
& +c^{6} d^{6}\left(b^{2}-d^{2}\right)\left(b^{2}-c^{2}\right) \pm 2 a b c \sqrt{\left(b^{2}-a^{2}-d^{2}\right)\left(b^{2} d^{2}-2 a^{2} d^{2}-2 c^{2} d^{2}+2 a^{2} c^{2}\right)} \\
& \left.\times\left[\left(d^{2}+c^{2}\right)\left(d^{2}-c^{2}\right)^{2} a^{4}+2 c^{2} d^{2}\left(c^{2} b^{2}-b^{2} d^{2}+c^{4}+d^{4}\right) a^{2}+c^{4} d^{4}\left(d^{2}+c^{2}-2 b^{2}\right)\right]\right\} \\
& /\left[a^{4} d^{4}+\left(a^{2}+d^{2}\right)^{2} c^{4}+2 a^{2} c^{2} d^{2}\left(a^{2}+d^{2}-2 b^{2}\right)\right]^{2} \\
m_{\tilde{\chi}_{2}^{0}}^{2}= & m_{\tilde{l}_{R}}^{2}\left(a^{2}+m_{\tilde{l}_{R}}^{2}-m_{\tilde{\chi}_{1}^{0}}^{2}\right) /\left(m_{\tilde{l}_{R}}^{2}-m_{\tilde{\chi}_{1}^{0}}^{2}\right) \\
m_{\tilde{q}_{L}}= & m_{\tilde{\chi}_{1}^{0}}+b
\end{aligned}
$$

For a given set of endpoint measurements the inversion formulae of most regions will return unacceptable masses. In some regions the masses returned will be in contradiction with the presupposed hierarchy, $m_{\tilde{q}_{L}}>m_{\tilde{\chi}_{2}^{0}}>m_{\tilde{l}_{R}}>m_{\tilde{\chi}_{1}^{0}}$, in some there will even be negative or imaginary masses. Such solutions must be discarded. If the masses returned by the inversion formulae of a region $(i, j)$ do obey the hierarchy, they constitute a physical solution if they themselves belong to $(i, j)$. Solutions which do not satisfy this last constraint will be referred to as 'unphysical'. In principle it should be possible to construct for each region a number of conditions on the endpoint values which, beforehand, would tell if the inversion formulae would return a physical solution or not. Such conditions will however become quite complicated, and have not been sought in this study. Instead the more straightforward approach is taken: try the inversion formulae of each region, then discard any unacceptable solutions.

Originating from realisation (4) of $m_{q l l}^{\max }$, see Eq. (4.4), where masses appear unsquared, the inversion formulae of region $(4, j)$ in principle come in two versions, the difference being a sign within one of the mass expressions. In regions $(4,1)$ and $(4,2)$ it turns out that only one of the signs returns masses in the appropriate region. In Eqs. (4.27) and (4.29) the physical sign is therefore chosen. For (4,3) both versions must be considered.

Once the required endpoints are measured and the inversion formulae used to determine the masses, we encounter a delicate feature of the entire method of obtaining masses from 
endpoints. While the endpoints are given by single-valued functions of the masses, albeit with different expressions for different mass regions, the inverse is not true. A given set of endpoint values can in principle correspond to several sets of mass values. This is equally true for the numerical fit method, and has not received much attention previously (see, however, Ref. 32]). This complication will be faced in Section 6 .

\section{5. 'Data' generation and reconstruction}

\subsection{Event generation}

The SPS points are defined by the low-energy MSSM parameters produced by ISAJET 7.58 [30], given a set of high-energy input parameters. In our analysis PYTHIA 6.2 [44] with CTEQ 5L 45] is used to generate the Monte Carlo sample. ${ }^{3}$ To allow for this the low-energy parameters from ISAJET are fed into PYTHIA via the standard interface. PYTHIA in turn calculates the decay widths and cross-sections. Each event produced is passed through ATLFAST 2.60 [47, a fast simulation of the ATLAS detector. In ATLFAST the output particles of PYTHIA are mapped onto a grid of 'calorimetric cells' of a given spacing in pseudorapidity $\eta$ and azimuth angle $\phi$. Next, the cells are combined into clusters, after which particle identification takes place, including smearing of the four-momenta according to particle type. Jets are built by a cone algorithm with $\Delta R=0.4$, where $\Delta R=\sqrt{(\Delta \eta)^{2}+(\Delta \phi)^{2}}$. Acceptance requirements are imposed: $|\eta|<2.5$ for $e / \mu$ and $|\eta|<5$ for jets as well as $p_{T}>5 / 6 / 10 \mathrm{GeV}$ for $e / \mu /$ jets. Leptons are marked as isolated if there is no other cluster within a distance $\Delta R=0.4$ of the lepton, and if additional energy inside a cone $\Delta R=0.2$ is below $10 \mathrm{GeV}$. While ATLFAST captures quite well the main features of the full simulation, some important effects are left out. Lepton identification efficiencies are not parametrized. A conventional $90 \%$ efficiency per lepton is therefore included by hand in the analysis. Also, the possibility of misidentifying a jet as a lepton is absent in the fast simulation, and has not been included in our analysis. The effect of pileup on the jet energy resolution is accounted for in ATLFAST when run at high luminosity, as in this analysis, but pile-up events have not been simulated, and the underlying event is probably too 'slim'. However, as the selection criteria on jets and leptons are quite hard, we do not expect a more realistic detector simulation to change the results very much. Nevertheless, the numbers quoted at the end of this section should be validated with these effects included.

The signature of a signal event is two opposite-sign same-flavour (SF) leptons, considerable missing $p_{T}$ from the escaping LSPs, and at least two hard jets, one from the signal chain, the other from the decay of the squark nearly always present in the other decay chain. The most important Standard Model process to have the same features as the signal, is $t \bar{t}$ production. Also $W / Z$ together with jets, one of which is a $b$-jet, can mimic the signal, and in combination with the underlying event, pile-up and detector effects, other processes will also now and then result in the given signature. Together with $t \bar{t}$ we therefore include QCD, $Z / W+$ jet as well as $Z Z / Z W / W W$ production. No $K$-factors have been used.

\footnotetext{
${ }^{3}$ The main parts of the analysis have been confirmed with HERWIG [46], see [28].
} 
The precuts (not tuned) used to isolate the chain are the following,

- At least three jets, satisfying: $p_{T}^{\text {jet }}>150,100,50 \mathrm{GeV}$

- $E_{T, \text { miss }}>\max \left(100 \mathrm{GeV}, 0.2 M_{\text {eff }}\right)$ with $M_{\text {eff }} \equiv E_{T, \text { miss }}+\sum_{i=1}^{3} p_{T, i}^{\text {jet }}$

- Two isolated opposite-sign same-flavour leptons ( $e$ or $\mu$ ), satisfying $p_{T}^{\text {lep }}>20,10 \mathrm{GeV}$

The QCD background is cut away by the requirement of two leptons and of considerable missing $p_{T}$. For the processes involving $Z$ and $W$ the requirement of high hadronic activity together with the missing $p_{T}$ removes nearly all events. After the rather hard cuts listed above, the Standard Model background consists of approximately $95 \% t \bar{t}$.

\subsection{Different Flavour (DF) subtraction}

At this stage the main background events, in addition to $t \bar{t}$, will come from other SUSY processes which have two opposite-sign same-flavour leptons. It is useful to distinguish between two types. The first, which will also be referred to as 'lepton-correlated', produces correlated leptons, e.g. leptonic decay of $Z$. In these processes the leptons always have the same flavour. The other type produces uncorrelated leptons which need not be of the same flavour. Typically the uncorrelated leptons are produced in different decay branches within the same event. Lepton universality implies that electrons and muons are produced in equal amounts (apart from negligible mass effects). This means that for events which produce uncorrelated leptons, there should be as many opposite-sign different-flavour (DF) lepton pairs produced as there are opposite-sign same-flavour lepton pairs, and their event characteristics should be the same. The same-flavour leptons are already part of the selected events. If one then subtracts the different-flavour events from the total same-flavour sample, this corresponds statistically to removing the non-signal same-flavour events which come from uncorrelated leptons. The residual of the subtraction is a larger statistical uncertainty in the new signal distribution. Clearly the different-flavour subtraction is a very effective tool which takes care of both $t \bar{t}$ and most of the SUSY background. Only processes with correlated leptons remain.

In a more detailed study one would need to correct for the different acceptances of electrons and muons as functions of $\left(p_{T}, \eta, \phi\right)$ in the different-flavour subtraction. Here we have assumed equal acceptance for electrons and muons, which even for a fast simulation is only approximately true. Also we have assumed that the efficiencies for reconstructing two same-flavour and two different-flavour leptons are the same. For close leptons this is not the case in the ATLFAST reconstruction. This will have some visible effects in the different-flavour-subtracted distributions, see below.

Fig. 11 shows the different-flavour subtraction for the $m_{l l}$ (top) and $m_{q l l}$ (bottom) distributions at points $(\alpha)$ (left) and $(\beta)$ (right). (The jet used in the $m_{q l l}$ distribution is the one of the two $p_{T}$-hardest jets which gives the smaller value of $m_{q l l}$.) All the plots have the same colour code. In red the same-flavour ('SF') distribution is shown by solid curve, the different-flavour ('DF') one by dashed curve. Their difference, the differentflavour-subtracted distribution ('SF-DF'), is shown in black with errors. The curve in blue 

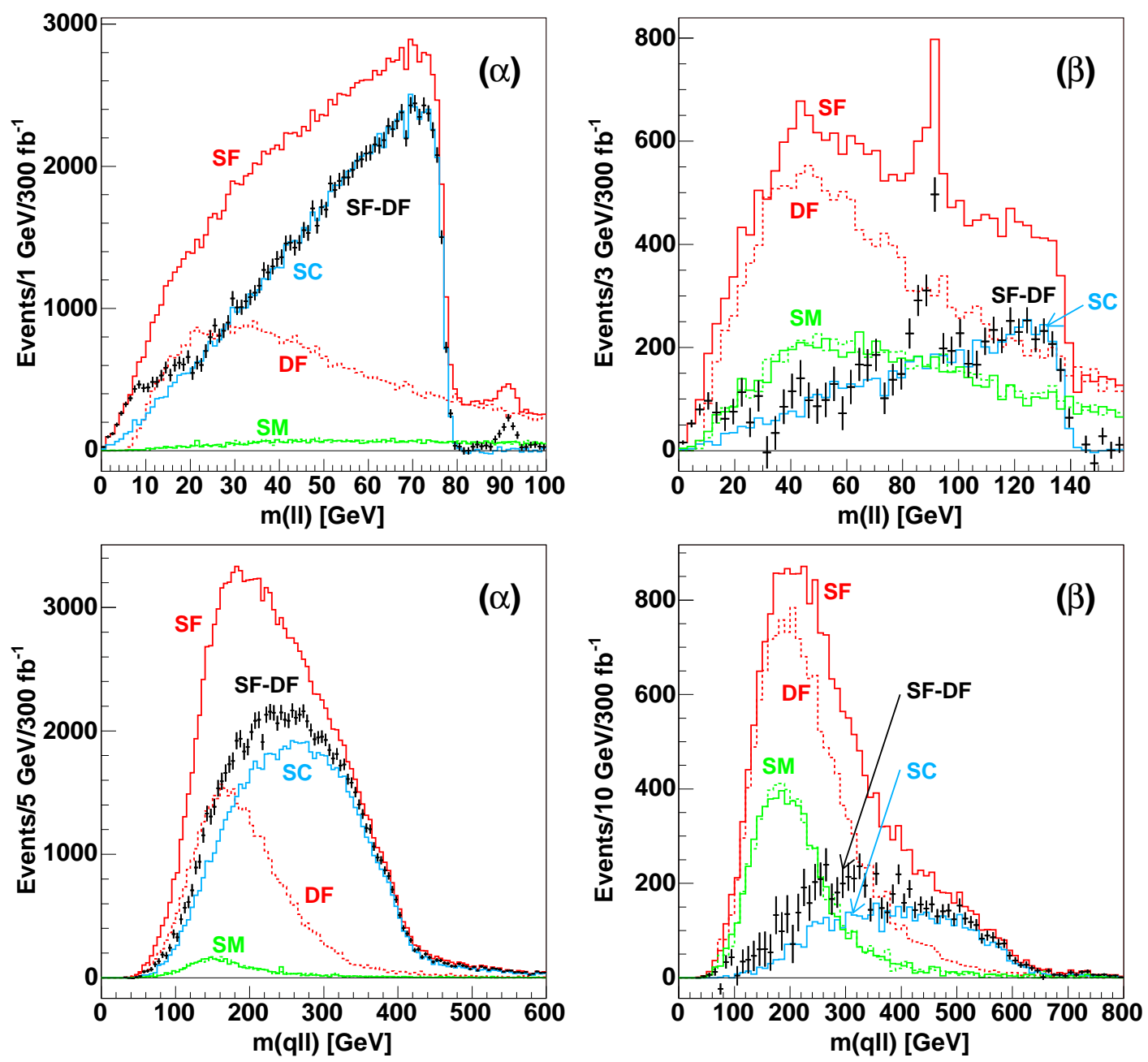

Figure 11: Different-flavour subtraction for $m_{l l}$ and $m_{q l l}$ at $(\alpha)$ (left) and ( $\beta$ ) (right). The solid/dashed red curves are the same-flavour ('SF')/different-flavour ('DF') distributions. In black, their difference, the different-flavour-subtracted distribution ('SF-DF'), is shown with error bars. The blue curve shows the part of the subtracted distribution which contains a signal chain ('SC'). The solid/dashed green curves ('SM') give the Standard Model part (completely dominated by $t \bar{t}$ ) of the same-flavour/different-flavour distributions. They are statistically equal and will cancel each other.

shows the part of the different-flavour-subtracted distribution which contains a signal chain ('SC'). The reason why the blue distribution of $m_{\text {qll }}$ does not have the form of the theory distribution in Fig. 10, is that the jet is only correctly selected in roughly half of the cases. In solid/dashed green the Standard Model contributions to the same-flavour/different-flavour distributions are shown ('SM'). They are statistically identical and will cancel each other through the different-flavour subtraction.

If the samples contained only background events with uncorrelated leptons (and the different-flavour-subtraction procedure removed all of these), the different-flavour-subtracted distribution should fall exactly on top of the blue line. When this does not happen, it implies that the sample also contains background events with correlated leptons. The 
$Z$-peaks in the $m_{l l}$ distribution at both $(\alpha)$ and $(\beta)$ are obviously of this type. The $Z$ 's stem predominantly from the decay of heavier gauginos into lighter ones. At $(\alpha) \sim 80 \%$ of the peak comes from the heavy gauginos $\tilde{\chi}_{3}^{0}, \tilde{\chi}_{4}^{0}$ and $\tilde{\chi}_{2}^{ \pm}$. At $(\beta) \tilde{\chi}_{2}^{0}$ is sufficiently heavy to decay into $\tilde{\chi}_{1}^{0} Z$ and is responsible for $\sim 40 \%$ of the peak. The rest involves the heavy gauginos.

For $m_{l l} \lesssim 20 \mathrm{GeV}$ a bump is visible in the different-flavour-subtracted distribution. This excess turns out to come mainly from lepton-uncorrelated events, predominantly of the type $\tilde{\chi}_{2}^{0} \rightarrow \tilde{\tau}_{1} \tau$ which is abundant in the two scenarios, and where the taus decay leptonically. Since a pair of taus produce same-flavour and different-flavour leptons in identical amounts, the different-flavour subtraction should take care of this background. When it does not, it is because of an asymmetry in the reconstruction algorithm of ATLFAST which accepts close same-flavour leptons at a higher rate than close different-flavour leptons. Since small $\Delta R$ between the leptons means small $m_{l l}$ values, the bump appears at low $m_{l l}$. Such a reconstruction asymmetry may also have noticeable effects in other distributions, e.g. $m_{q l l}$, for which the maximum values in our two mass scenarios appear for parallel leptons. A dedicated study of the impact detector effects of this type may have on the endpoint determinations may be worthwhile.

In the $m_{q l l}$ distributions there is an excess of events (compared with the blue line) at lower masses. Some of this excess comes from the lepton-uncorrelated events in the $m_{l l}$ bump. Typically these land at low $m_{q l l}$ values. The main contribution to the excess is however from $\tilde{\chi}_{2}^{0}$ 's which decay sleptonically, but which originate from $\tilde{q}_{R}, \tilde{t}_{1}, \tilde{g}$ or the heavier gauginos.

At $(\beta)$ two effects complicate the endpoint measurements. One is a reduction of the SUSY cross-section by one order of magnitude relative to $(\alpha)$. This allows for the Standard Model background to have a larger impact, see the green curves in Fig. 11. Since this background (practically only $t \bar{t}$ ) consists of uncorrelated leptons, it is dealt with by the different-flavour subtraction. It leaves however an increased statistical uncertainty in the resulting distribution. The other effect at $(\beta)$ is a reduction in the signal branching ratio, mainly from a reduced $\operatorname{BR}\left(\tilde{\chi}_{2}^{0} \rightarrow \tilde{l}_{R} l\right)$, see Fig. 9. As a result also the SUSY background becomes larger relative to the signal.

While the Standard Model background is practically negligible at $(\alpha)$, it is of similar size as the SUSY background at $(\beta)$. For the $m_{q l l}$ distribution both background types are important only at low values, a fair distance away from the kinematical maximum value. The same is true for the $m_{q l}$ distributions. The endpoint measurements are therefore only

minimally affected by the background. An exception is the $m_{q l l\left(\theta>\frac{\pi}{2}\right)}$ distribution, where a minimum is measured.

\subsection{Selection cuts}

A fair fraction of the events which pass the precuts, $\sim 30 \%$ for $(\alpha)$ and $\sim 20 \%$ for $(\beta)$, have more than two leptons. Each opposite-sign lepton pair which satisfies $p_{T}>20,10 \mathrm{GeV}$ is used, either in the same-flavour or the different-flavour distribution. As usual, the differentflavour subtraction takes care of the incorrect combinations. 

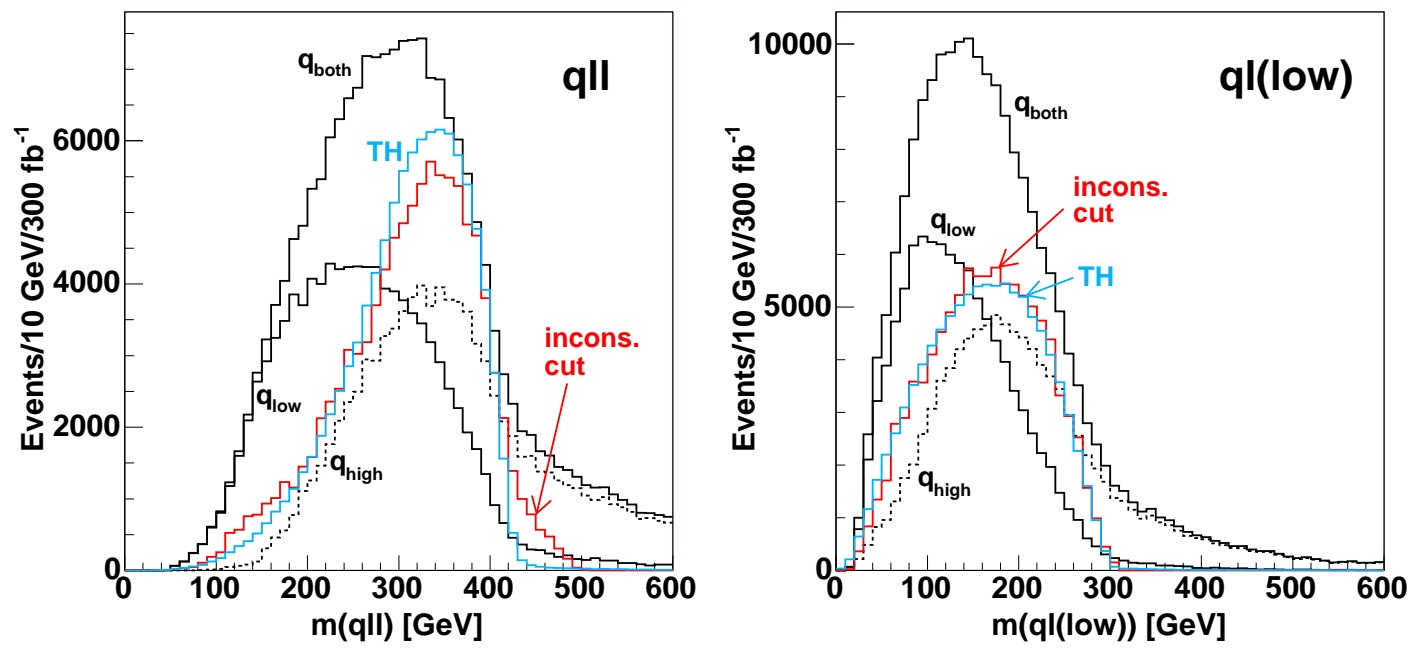

Figure 12: Quark selection cuts for $m_{q l l}$ (left) and $m_{q l(\text { low })}$ (right). Left: The two lower black curves in solid and dashed show the $m_{q_{\mathrm{low}} l l}$ (' $q_{\mathrm{low}}$ ') and $m_{q_{\mathrm{high}} l l}\left({ }^{\prime} q_{\mathrm{high}}\right.$ ') distributions. The highest black curve shows the sum of the two, $m_{q_{\mathrm{both}} l l}\left({ }^{\prime} q_{\mathrm{both}}{ }^{\prime}\right)$. Notation is defined in the text. Blue: theory distribution ('TH'). Red: inconsistency cuts ('incons. cut') have been applied on $m_{q l(\text { low })}$ and $m_{q l(\text { high) }}$ (the resulting distribution is scaled up). Right: An inconsistency cut is applied on $m_{q l l}$. The jet chosen for $m_{q_{\text {low }} l l}$ is the one used in $m_{q_{\text {low }} l(\text { low })}$ etc. The inconsistency cut is seen to work very well for the $m_{q l(\text { low })}$ distribution.

The selection of the jet to go with the two leptons is more difficult. In the precuts at least three jets are required. If the squarks are considerably heavier than $\tilde{\chi}_{2}^{0}$, and the gluinos are not very much heavier than the squarks, then the two $p_{T}$-hardest jets are expected to come from the decay of the two squarks present in nearly all events. (More specifically we need $m_{\tilde{q}}^{2}-m_{\tilde{\chi}_{2}^{0}}^{2}$ somewhat larger than $m_{\tilde{g}}^{2}-m_{\tilde{q}}^{2}$.) Along the entire SPS 1a line this is the case. The third jet is then expected to come from a gluino decay. Specifically, both for $(\alpha)$ and $(\beta)$ the correct quark is one of the two $p_{T}$ hardest in $94 \%$ of the cases at the parton level. It is therefore reasonable to consider only these two jets.

In a realistic setting all possible combinations will be investigated, along with all kinds of precuts. However, whatever the mass situation, one will not be able to select the correct jet by some simple cut. Combinatorial background from the jet selection procedure is something one will have to live with.

In Fig. 12 (left) the different-flavour-subtracted $m_{q l l}$ distribution at $(\alpha)$ is shown for various jet selections. The two lower black curves are obtained by selecting from the two $p_{T}$-hardest jets the one which gives the smaller (solid) and larger (dashed) value of $m_{q l l}$, for simplicity referred to as $m_{q_{\text {low }} l l}$ (' $q_{\text {low }}$ ') and $m_{q_{\text {high }} l l}$ (' $q_{\text {high }}$ '), respectively. The highest black curve shows the sum of the two, $m_{q_{\mathrm{both}} l l}$ (' $q_{\mathrm{both}}$ '). In blue and marked 'TH', with the same normalisation as $m_{q_{\text {low }} l}$, is the parton level theory distribution, as generated by PYTHIA so that it contains widths, production biases etc.

It is possible to get a reasonable first estimate of the endpoint both from $m_{q_{\text {low }} l l}$ and $m_{q_{\mathrm{both}} l l}$. The shape of the theory distribution is however not reproduced by any of the three experimental distributions (in black), which is quite natural since they all contain 
both combinatorial and lepton-correlated background, and two of them are also biased by the jet selection procedure. For $(\alpha)$ the lepton-correlated background comes mainly from sleptonically decaying $\tilde{\chi}_{2}^{0}$ 's that do not have a $\tilde{q}_{L}$ or $\tilde{b}$ as a parent. As can be seen from Table 3 together with Fig. 9, $\tilde{\chi}_{2}^{0}$ comes from $\tilde{q}_{L}$ or $\tilde{b}$ in $78 \%$ of the times. If, for a signal event, we assume $50 \%$ chance of picking the correct jet, a different-flavour-subtracted distribution will consist of $39 \%$ signal, $39 \%$ combinatorics and $22 \%$ lepton-correlated background. For $(\beta)$ the fractions are $35 \%, 35 \%$ and $30 \%$. For these numbers $\tilde{\chi}_{2}^{0}$ is assumed to be the only source of lepton-correlated background.

We have investigated methods to remove or model this combinatorial background. For example, the sample may be purified [25] by using events where only one of the jets can reasonably be assigned to the signal quark. Consider the case where a measurement of $m_{q l l}^{\max }$ has been made, and one wants to now measure $m_{q l(\mathrm{low})}^{\max }$. In constructing the $m_{q l(\text { low })}$ distribution we must choose one of the two highest $p_{T}$ jets, and with no other input will choose the wrong jet $50 \%$ of the time, giving a combinatorial background. However, in some events, one of the jets will be such that its intepretation as the signal quark would contradict the previous measurement of $m_{q l l}^{\max }$, i.e. using it to construct $m_{q l l}$ would give $m_{q l l}>m_{q l l}^{\max }$. In these events, we can be reassured that in choosing the other jet we have chosen correctly. If neither of the jets in the event gives $m_{q l l}$ in contradiction with the previous measurement, then we cannot be certain that we have the correct jet, and therefore discard the event. In other words, we only use events where only one choice of signal quark is consistent with other endpoint measurements. This allows one to build a purified sample where for signal events the wrong jet is only chosen if the correct jet is not one of the two $p_{T}$-hardest jets. There will however still be background events in the sample.

The red curve of Fig. 12 (left) is constructed from the $m_{q l l}$ distribution by means of 'inconsistency cuts' on $m_{q l(\text { low })}$ and $m_{q l \text { (high) }}$ where we insist that only one of the two highest $p_{T}$ jets has $m_{q l \text { (low) }}<310 \mathrm{GeV}$ and $m_{q l \text { (high) }}<390 \mathrm{GeV}$. Approximately $1 / 4$ of the events pass these cuts; the resulting curve is normalised to have the same number of events as the $m_{q_{\text {low }} l l}$ distribution. The similarity with the blue theory curve has improved, but is still not excellent. In particular there is a large tail towards higher values, which in practice makes it less useful for accurate endpoint determination.

In this respect a better result is obtained for the $m_{q l(\text { low })}$ distribution, shown in Fig. 12 (right) with an inconsistancy cut requiring only one jet with $m_{q l l}<440 \mathrm{GeV}$, and normalisation as $m_{q_{\text {low }} l(\text { low })}$. The red curve matches quite well with the theory curve, and there is no disturbing tail. In fact the red curve is the one we later use to estimate the endpoint for this distribution.

While it is certainly reassuring to regain the theoretical distribution, it is still not clear what is the best way to obtain an accurate measurement of the endpoints. One would probably combine many different methods, looking for convergence as well as inconsistencies. For instance, even though we should choose not to fit the distribution from the inconsistency cut, since it has less statistics, it teaches us that a linear fit to $m_{q_{\text {low }} l \text { (low) }}$ from 150 to $300 \mathrm{GeV}$, which might otherwise seem reasonable, would not at all be a fit to the signal since it is close to linear only in the region $240-300 \mathrm{GeV}$. 
Another approach to obtaining a distribution closer to the original one relies not on purification of the actual sample, but on finding an estimate for the background, combinatorial and/or lepton-correlated, and then subtracting it from the original distribution. Statistics may be better preserved in this way, but systematics are introduced. Some attempts have been made with 'mixed events', i.e. the combination of the lepton pair from one event with jets from other events. The idea is that since the lepton and the jet sectors of mixed events are necessarily uncorrelated, they may mimic both types of background, lepton-correlated and combinatorial, where also the lepton pair is only weakly correlated with the jet.

A first complication of this method is encountered when the four-vectors of different events are to be combined into mixed events. What rest frame is the appropriate one? This already points to the inexact nature of such a method. Its performance is however promising, although somewhat variable, as will be demonstrated later in this section.

The mixed event sample is constructed from the events which make up the distributions, i.e. those which pass the precuts listed in Sect. 5.1. An additional requirement of having exactly two opposite-sign leptons is imposed to avoid ambiguities in the lepton sector. No major differences are however expected from leaving out this constraint. Each event then provides two leptons, uniquely defined because of the additional cut, and its two $p_{T}$-hardest jets. The four-vectors of the selected leptons and jets are here taken in the rest frame of the lepton pair. Due to the prominent role of the lepton pair, this choice is not unreasonable. On the other hand it is not unique. Both the laboratory frame, the rest frame of the entire event or the rest frame of the leptons together with the two $p_{T}$-hardest jets are viable candidates. A dedicated study of the characteristics of these different possibilities would be worthwhile.

The mixed sample is then constructed by consecutively combining the lepton sector of one event with the jet sector of another. In our study each lepton sector was combined with the jet sector of five other events. By increasing this number, very high statistics can be obtained for the mixed sample. For each combination of events all relevant masses were constructed, $m_{q_{\text {low }} l}, m_{q_{\text {high }} l l}, m_{q_{\text {both }} l}, m_{q_{\text {low }} l(\text { low })}$ etc.

Mixed samples for same-flavour and different-flavour events are constructed separately. In the end their difference is taken, in line with the previously described different-flavour subtraction. The resulting mixed distributions are the ones used later in this section.

\subsection{Multiple squark masses}

The theory distributions shown in Fig. 10 are only for one squark mass per scenario. In

reality four different squark masses, $m_{\tilde{d}_{L}} / m_{\tilde{s}_{L}}, m_{\tilde{u}_{L}} / m_{\tilde{c}_{L}}, m_{\tilde{b}_{1}}$ and $m_{\tilde{b}_{2}}$ will contribute to the distribution. The blue theory distribution of Fig. 12 is therefore the sum of four separate distributions, each with a different endpoint, different normalisation and similar though not identical shapes.

While jets from the three lightest quarks, and for most purposes also from the $c$-quark, will be indistinguishable in ATLAS, jets from $b$-quarks will be identified with a certain probability. The expected rejection factors for incorrectly identifying jets from lighter quarks and gluons as $b$-jets, are for high luminosity operation given in the ATLAS TDR 
[20], Fig. 10-41. Low/high $b$-tagging efficiencies come with high/low rejection factors and allow for high-purity $b /$ non- $b$-samples, respectively. The higher the purity, the smaller the sample. In this analysis we have used the following simplistic $b$-tagging prescription for both purposes: For a $b$-tagging efficiency of $50 \%$ the rejection factors against jets from gluons/three lightest jets and from $c$-jets are set to 100 and 10, respectively.

With $b$-tagging one can to a certain extent separate the $\tilde{q}_{L}$ and the $\tilde{b}$ distributions, thus opening for a disentanglement of the squark masses. Nevertheless, even though a high purity separation has been accomplished, each of the two distributions will still contain contributions from two squark masses. Typically a kink can be observed at the position of the lowest endpoint. For mSUGRA scenarios $\tilde{d}_{L} / \tilde{s}_{L}$ and $\tilde{u}_{L} / \tilde{c}_{L}$ only differ by a few $\mathrm{GeV}$, so the kink will appear very near the end of the distribution. Since the proton contains more $u$ than $d$-quarks, $\tilde{u}_{L}$ will be produced at a higher rate than the heavier $\tilde{d}_{L}$. This reduces further the visibility of the kink. Then with the general smearing due to physics and detector effects in addition to background near the endpoint, it may be very difficult to identify such a kink. In case of the two $b$-squarks the separation will be larger. Whether it is possible to identify it or not depends on the rate of $\tilde{b}$ production as well as the level of impurity from $\tilde{q}_{L}$-events in the $b$-tagged distribution.

\subsection{Invariant mass distributions}

SUSY processes for $(\alpha)$ and $(\beta)$ as well as the Standard Model background have been produced for $300 \mathrm{fb}^{-1}$. This corresponds to 3 years at design (high) luminosity. The mass distributions of the available edges for SPS 1a $(\alpha)$ and $(\beta)$ are shown in Figs. 1314.

For all plots the black points with errors show the total different-flavour-subtracted distribution ('SF-DF'). Solid green marks the SUSY background ('SUSY'), and in green with error bars the Standard Model background ('SM') is shown. The solid blue curve then shows the original theory distribution ('TH'), normalised to the different-flavour-subtracted distribution. The fitted function appears in red. In cases where mixed events are used to model the background, the smooth function fitted to the high-statistics mixed-event sample is shown in dashed red. When additional distributions are plotted, they will be described in the accompanying discussion.

For each distribution the endpoint estimation will be discussed. In most cases the edges are fitted to a straight line in combination with a simple background hypothesis, and in some cases convoluted with a Gaussian distribution. At $(\alpha)$ this procedure gives numbers in reasonably good agreement with the nominal values. At $(\beta)$, where the SUSY cross-section is much smaller and also the branching fraction of the signal is reduced, the estimated endpoint values depend more strongly on the fitting method chosen. To control and reduce this systematic effect, a better understanding of the whole chain is required; physics effects, detector effects, multiple masses at different rates, background, precuts. After some years of LHC operation one can expect these issues to be understood sufficiently that the systematics of endpoint estimation is controlled and corrected for in the fitting procedure, up to some small uncertainty.

If this is achieved, it is the statistical error of the endpoint values, in combination with the uncertainty on the absolute energy scale, expected to be $1 \%$ for jets and $0.1 \%$ for 
leptons, which determine the precision at which masses can be obtained for the different scenarios.

In the discussion of the distributions we therefore have the main focus on the statistical uncertainty of the endpoint determination, but are also concerned with the present magnitude of the systematic uncertainty. For some of the distributions it may seem bold to state that the systematic fit uncertainty will be reduced far below the statistical one. One shall however remember that once such distributions become available, a great effort will go into investigating them.

\section{SPS 1a $(\alpha)$}

$\boldsymbol{m}_{l l}$ : The $m_{l l}$ distribution at $(\alpha)$ is shown in Fig. 13 (top left). It is very close to the expected triangular shape. To the right of the endpoint the $Z$ peak is clearly visible. These $Z$ 's come from SUSY decays. The bump at low $m_{l l}$ is discussed in Sect. 5.2.

The distribution is fitted with a straight line convoluted with a Gaussian,

$$
g\left(m_{l l}\right)=\left\{\begin{array}{ll}
A m_{l l}, & m_{l l}<m_{l l}^{\max } \\
0, & m_{l l}>m_{l l}^{\max }
\end{array} \quad, \quad f\left(m_{l l}\right)=\frac{1}{\sqrt{2 \pi} \sigma} \int_{0}^{\infty} d m_{l l}^{\prime} g\left(m_{l l}^{\prime}\right) e^{-\frac{\left(m_{l l}-m_{l l}^{\prime}\right)^{2}}{2 \sigma^{2}}}\right.
$$

Both the endpoint, the width of the Gaussian and the normalisation are kept free in the fit, resulting in an endpoint value of $76.72 \mathrm{GeV}$ at a statistical error of $0.04 \mathrm{GeV}$. Compared to the nominal value this is $0.35 \mathrm{GeV}$ too low. When a more appropriate model for loss of lepton energy is implemented in the fit function, such a systematic shift is expected to be reduced to the level of the statistical error or below. The fit results are quite stable with respect to variations in bin size and range of fit. We thus arrive at the well-known result that the $m_{l l}$ endpoint is expected to be estimated to high precision.

$\boldsymbol{m}_{\boldsymbol{q} l l}$ : As for all cases where jets are involved, the $m_{q l l}$ distribution comes in many versions, depending on the jet selection procedure as well as the use of $b$-tagging information. Plotted in Fig. 13 (top right) is $m_{q_{\text {low }} l l}$ (see Sect. 5.3) with no $b$-veto. Mixed events have been used in an attempt to model the background. A $6^{\text {th }}$ degree polynomial was first fitted to the high statistics mixed event sample. Then, with the normalisation kept free, the polynomial was combined with a straight line for the signal part. This procedure returns $m_{q l l}^{\max }=427.7(0.9) \mathrm{GeV}$, statistical error in parenthesis. The nominal value is $425.9 / 431.1 \mathrm{GeV}$ for $\tilde{u}_{L} / \tilde{d}_{L}$. Alterations to the bin size and fit range has a $1-2 \mathrm{GeV}$ effect on the fit value, but the statistical error remains the same.

The dashed red line shows the shape of the mixed event sample. It matches the $m_{q_{\text {low }} l} l$ distribution very well at values beyond the endpoint, justifying its use, and is sufficient to obtain a reasonable endpoint fit. Similar results can be found by modeling the background tail with an exponential or a polynomial. If, at some later stage, the entire distribution is to be compared with the theory distribution, the size of the background must be known for any $m_{q l l}$ value. An exponential or a polynomial based on the upper tail can never be a good background model for values somewhat below the edge. It is here the mixed events may have a role to play. In the particular case of the $m_{q_{\text {low }} l l}$ distribution, the mixed sample actually appears to be a good background estimate at all values. For $m_{q_{\mathrm{both}} l l}$ and some 

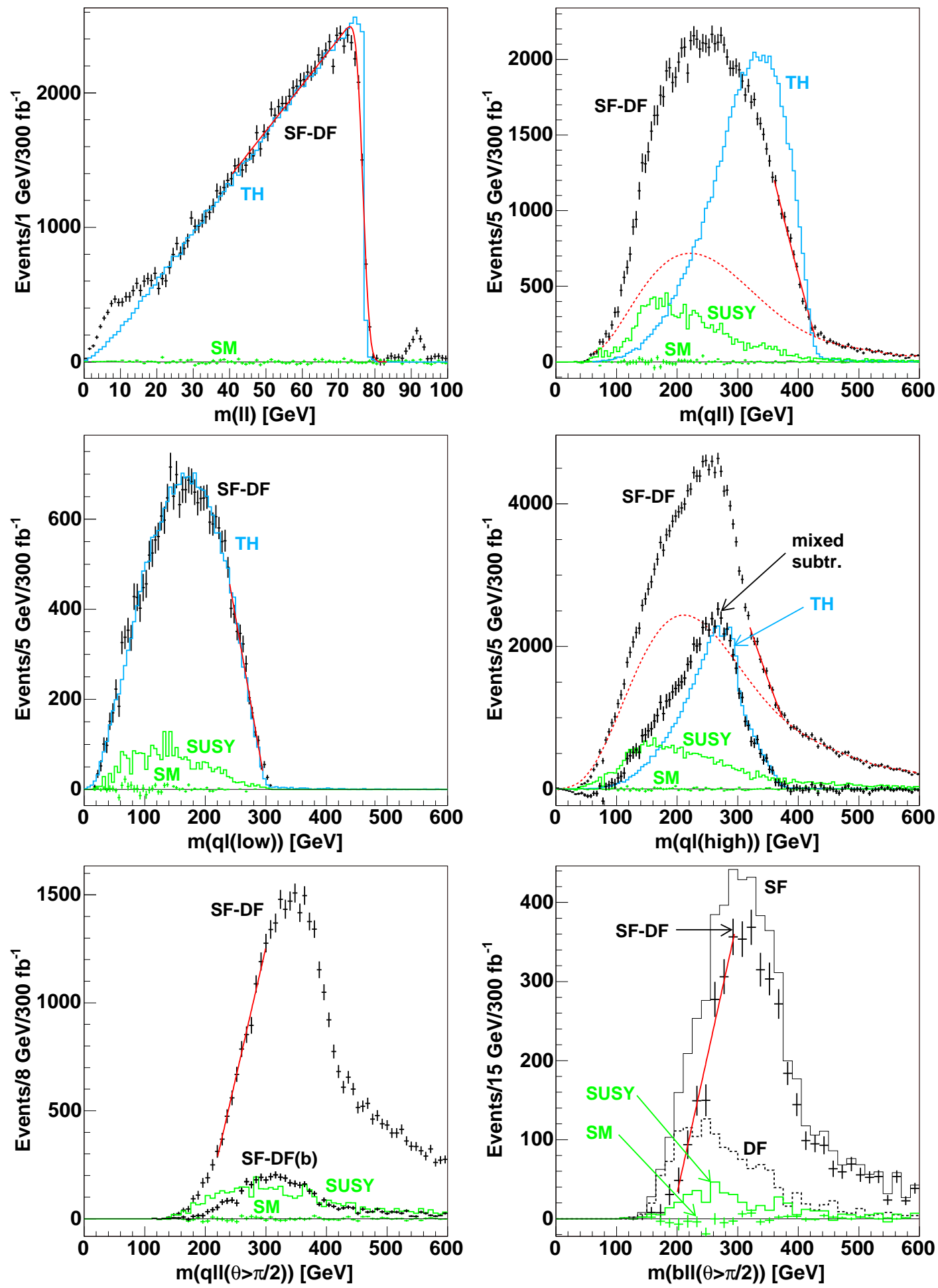

Figure 13: Invariant mass distributions for SPS 1a $(\alpha)$. See the text for details.

other distributions, it does not do so well. Nevertheless, there are many ways to construct the mixed sample. Further study of the method seems worthwhile. 
It is tempting to suggest that it is mainly the $\tilde{u}_{L} / \tilde{c}_{L}$ endpoint we measure, and that the extra events just above the endpoint (see the figure), make up the hard-to-detect kink from the $\tilde{d}_{L} / \tilde{s}_{L}$ edge. At the present level of detail it is impossible to say if such an effect could be isolated to give an additional measurement for the heaviest mass. It does seem difficult, though, as the kink will be washed out by other effects, e.g. sparticle widths. At $(\alpha)$ the intrinsic width of $\tilde{u}_{L}$ is $5.3 \mathrm{GeV}$, see Fig. 6 (right), to be compared with $m_{\tilde{d}_{L}}-m_{\tilde{u}_{L}}=5.8 \mathrm{GeV}$. Also detector effects will result in a general smoothing of the distributions.

$\boldsymbol{m}_{\boldsymbol{q l}(\text { low })}$ : For $m_{q l(\text { low })}$ the mixed sample is fine above the endpoint, but overestimates for lower masses. While the edge can be fitted and the endpoint measured by a mixed sample subtraction, due to its good behaviour in the edge region, we have here instead used the inconsistency cut $m_{q_{\mathrm{high}} l}>440 \mathrm{GeV}$ to purify the sample, Fig. 13 (middle left). The ability of the inconsistency cut to bring the distribution very close to the original one, was already discussed in relation to Fig. 12 .

For a zero background hypothesis a straight line fit gives $m_{q l(\text { low })}^{\max }=300.7(0.9) \mathrm{GeV}$, to compare with the nominal $298.5 / 302.1 \mathrm{GeV}$ for $\tilde{u}_{L} / \tilde{d}_{L}$. If the few bins around the endpoint are also included, the value increases by $1-2 \mathrm{GeV}$. Whether these high-mass events are signal or background is not easy to tell from the given distribution, since there is virtually no background structure to extrapolate from. This is because a 'consistency cut' has been imposed which requires $m_{q_{\text {low }} l l}<440 \mathrm{GeV}$, in accordance with the already measured $m_{q l l}$ endpoint. From Eq. (4.10) this implies $m_{q l(\text { low })}<440 / \sqrt{2} \mathrm{GeV}=311.1 \mathrm{GeV}$. The consistency cut takes away a large part of the background, but also has the effect that it becomes difficult to see what structure the $m_{q l \text { (low) }}$ background has. If the consistency cut is dropped, the usual background tail appears and can be modeled, although at the cost of a slight increase in the statistical error.

$\boldsymbol{m}_{\boldsymbol{q l}(\text { high })}$ : Following the same procedure as for $m_{q l l}$ the background of the $m_{q l(\text { high })}$ distribution was modeled by the mixed event sample. In Fig. 13 (middle right) the relevant distributions are shown. Also the result from subtracting the mixed sample is shown ('mixed subtr.'); the lower black points with error bars. This subtracted distribution follows the original theory distribution (blue) closely in the edge region, but overestimates at lower values.

In the range $m_{q l(\text { high }} \in(320,550) \mathrm{GeV}$ the endpoint was estimated to $374.0(2.0) \mathrm{GeV}$. The nominal value is $375.8 / 380.3 \mathrm{GeV}$ for $\tilde{u}_{L} / \tilde{d}_{L}$. At $(\alpha)$ the $m_{q l(\text { high })}$ edge consists of two parts. This is clear from the theory distribution (blue), but also in the reconstructed distributions (black). In the fit only the lowest near-linear stretch was used. It is clearly incorrect to apply a straight line for the whole edge, but if done, the statistical error would be reduced to $\sim 1 \mathrm{GeV}$. When a good signal function is at hand, the whole edge will be described. It may therefore be reasonable to expect a statistical error of $1 \mathrm{GeV}$ rather than $2 \mathrm{GeV}$.

$\boldsymbol{m}_{\boldsymbol{q l l}\left(\boldsymbol{\theta}>\frac{\pi}{2}\right)}$ : The $m_{q l l\left(\theta>\frac{\pi}{2}\right)}$ distribution differs from the previous ones in that a minimum is to be measured. This has two important consequences. One can be seen from Fig. 11 
(bottom left), even though this plot is for the unconstrained $m_{q l l}$ distribution. The leptonuncorrelated background, modeled by the different-flavour distribution (dashed red), sits mostly at low values, far away from any maximum edge, but quite near the $m_{q l l\left(\theta>\frac{\pi}{2}\right)}$ threshold. While this background type is removed (statistically) by the different-flavour subtraction, the price is an increased statistical uncertainty which directly will affect the statistical precision of the $m_{q l l\left(\theta>\frac{\pi}{2}\right)}^{\min }$ estimation.

The other consequence of measuring a minimum is related to the multiple squark masses. As noted earlier, the distributions can be purified using $b$-tagging information. For the measurements discussed so far we have considered the total sample. This is because the $\tilde{q}_{L}$-squarks are so much heavier than the $\tilde{b}$-squarks that the upper edge regions are practically free of $\tilde{b}$-events anyway. A $b$-veto has therefore no effect, at least not at our level of precision. For the threshold measurement it is the other way around. Here the threshold related to the lightest squark, $\tilde{b}_{1}$, will be the threshold for the total distribution. A $b$-veto may therefore be important in order to obtain the threshold for the non- $\tilde{b}$-events.

A third complication is that the edge is very non-linear, as can be seen from the theory distributions in Fig. 10. Also, the fact that this non-linearity is of the concave type, could make it very difficult to see where the edge ends and where smearing from various sources takes over. This is all the more relevant since the $\tilde{b}$-events come at a significantly smaller rate than $\tilde{q}_{L}$-events (ratio $\sim 25 \%$ ), and may therefore appear as just another contribution to the lower tail, not easily distinguishable from other effects.

The $m_{q l l\left(\theta>\frac{\pi}{2}\right)}$ distribution is shown in Fig. 13 (bottom left). The upper distribution ('SF-DF') shows the non- $b$-tagged $m_{q l l\left(\theta>\frac{\pi}{2}\right)}$ sample. The lower black points ('SF-DF $(b)^{\prime}$ ) mark the $b$-tagged sample. Only approximately $50 \%$ of the events which contain a $b$ are actually $b$-tagged. This means that the non- $b$-tagged distribution contains a 'hidden' $b$-sample, statistically similar to the 'visible' $b$-sample both in size and shape. (There would be a shift to the left, though, since in the reconstruction $b$-tagged jets have larger recalibration factors. This is not accounted for here.) Analogous to the different-flavour subtraction it is therefore possible to get the non- $b$-tagged distribution closer to the original $q$-distribution by subtracting the visible $b$-sample, possibly scaled up or down according to the $b$-tagging efficiency applied. A slightly different approach would be to use a much harder $b$-veto and no subtraction. The price would be a significant reduction in sample size.

However, the main problem in the threshold region is not the $b$-contamination, but the lepton-correlated SUSY background shown in solid green. It comes primarily from events where $\tilde{\chi}_{2}^{0}$ decays sleptonically but does not descend from $\tilde{q}_{L}$ or $\tilde{b}$. It is this background which needs appropriate modeling. The difficulty with having a concave theory distribution together with an unknown background is evident from the figure. It is really difficult to separate the background from the signal.

As a simple estimate of the statistical uncertainty of the edge position, a straight line fit somewhat away from the threshold was performed on the non-subtracted sample ('SF-DF'), returning a statistical error on the endpoint around $1.8 \mathrm{GeV}$. If the subtracted sample (subtracting 'SF-DF $(b)$ ') is used, the error increases. The actual endpoint obtained from such a simple zero-background hypothesis is of course incorrect. At present it seems a 
bit optimistic to expect the systematics of the fit to be dominated by the statistical error.

$\boldsymbol{m}_{b l l\left(\boldsymbol{\theta}>\frac{\pi}{2}\right)}$ : The $m_{b l l\left(\theta>\frac{\pi}{2}\right)}$ distributions are shown in Fig. 13 (bottom right). The differentflavour-subtracted distribution ('SF-DF') is shown in black with error bars. The sameflavour ('SF') and different-flavour ('DF') curves are also plotted. In the threshold region the same-flavour and different-flavour distributions are considerably larger than their difference, which necessarily gives large statistical uncertainties in the different-flavoursubtracted samples.

As for $m_{q l l\left(\theta>\frac{\pi}{2}\right)}$ more studies are required to control and reduce the systematic error induced by the background and the nonlinear theory curve. A linear fit was performed in the near-linear region to measure the precision with which the position of the edge can be found. Variations in the bin size and the fit range yield systematic errors within $1 \mathrm{GeV}$ and statistical error between 4 and $5 \mathrm{GeV}$.

$\boldsymbol{m}_{\boldsymbol{b l l}}, \boldsymbol{m}_{\boldsymbol{b l}(\text { low })}, \boldsymbol{m}_{\boldsymbol{b l}(\mathbf{h i g h})}$ : Given that the background can be controlled and a good signal function can be found, $m_{b l l\left(\theta>\frac{\pi}{2}\right)}$ is a good distribution since the lowest threshold is given by $\tilde{b}_{1}$, which also has a considerable rate. For the edges $m_{b l l}, m_{b l(\text { low) }}$ and $m_{b l(\text { high })}$ one problem is that it is $\tilde{b}_{2}$ which defines the outer endpoint for the $b$-distributions. Since this squark only contributes $\sim 22 \%$ of the $\tilde{b}$-events, the edge above the $\tilde{b}_{1}$ endpoint may look like background. (However, since we expect the heavier $\tilde{b}$-squark to come at a considerably smaller rate as it has less phase space for production, and also a smaller branching ratio to the wino-like $\tilde{\chi}_{2}^{0}$, due to its larger right-handed component, we can explicitly look for such a small tail-like edge.)

A more serious problem is the background from other SUSY events. Typically these have the decay Eq. (3.3) in one chain, providing two leptons, and $\tilde{g} \rightarrow \tilde{b} b$ or $\tilde{g} \rightarrow \tilde{t}_{1} t$ in the other, providing $b$-jets. The mass distributions of this background stretch sufficiently beyond the $\tilde{b}_{1}$ endpoints that the resulting edge structures become difficult to analyse. The positions of these three $b$-edges can typically be measured with a statistical precision of 3-4 GeV, but it remains unclear whether the systematics of the edge can be sorted out. Another approach could be to require a lower $b$-tagging efficiency for these distributions in order to get higher rejection factors and purer, although smaller, samples.

\section{SPS 1a $(\beta)$}

The mass distributions of $(\beta)$ are shown in Fig. 14. Throughout, the total samples have been used. No $b$-veto has been applied.

$\boldsymbol{m}_{l l}$ : The $m_{l l}$ distribution is shown in Fig. 14 (top left). It has the triangular shape with a $Z$-peak on top. Some datapoints in the $Z$-peak lie above the range of this plot, and can be viewed in Fig. 11 (top right).

As was also the case for $(\alpha)$ there is a bump at lower values. The distribution above the $Z$-peak was fitted with the same Gaussian-convoluted triangular shape as earlier, Eq. (5.1), giving $m_{l l}^{\max }=137.4(0.5) \mathrm{GeV}$ which is $0.5 \mathrm{GeV}$ lower than the nominal value. 

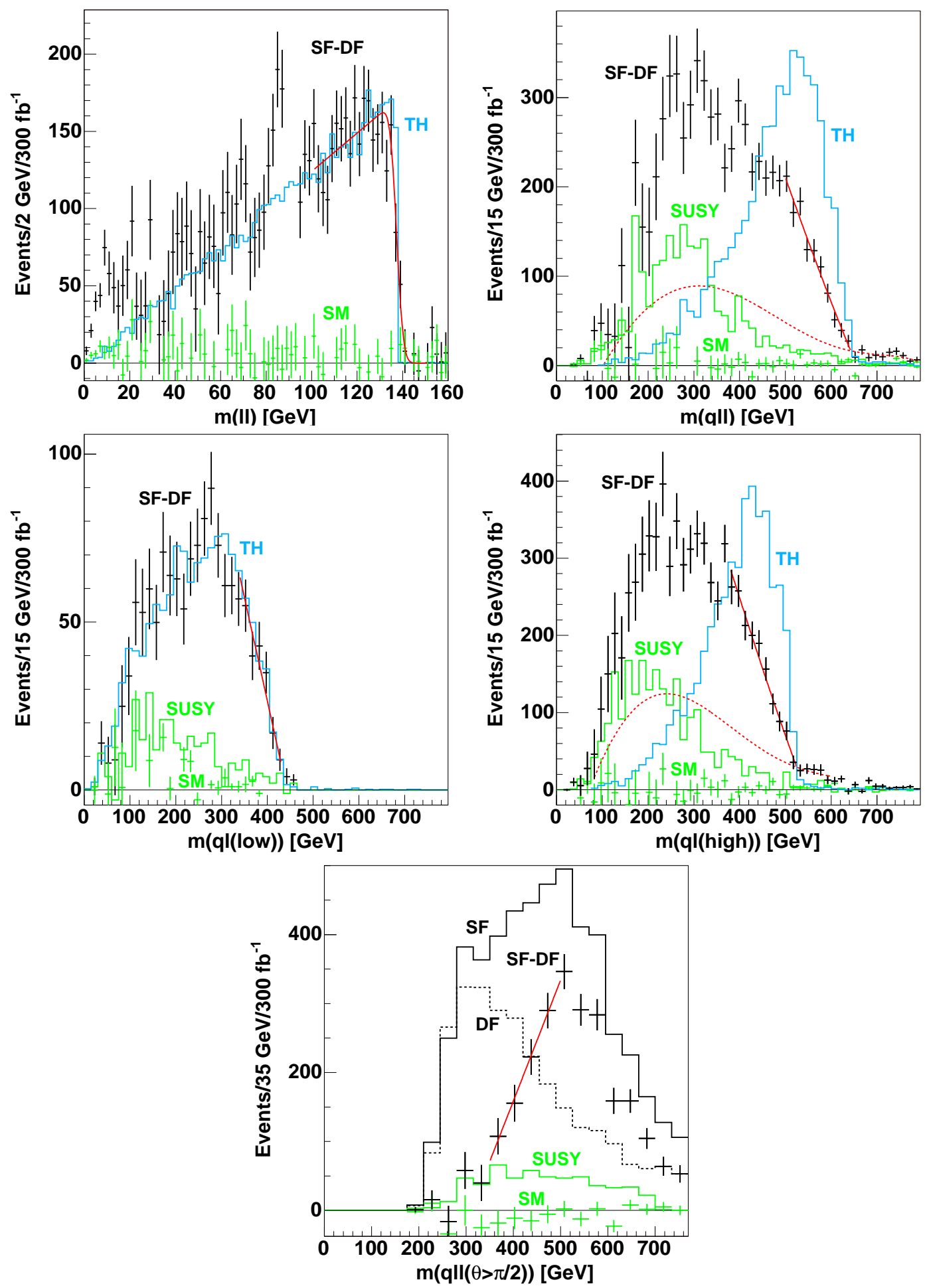

Figure 14: Invariant mass distributions for SPS 1a $(\beta)$. See the text for details.

$\boldsymbol{m}_{\boldsymbol{q} l l}$ : The $m_{q_{\text {low }} l l}$ distribution is shown in Fig. 14 (top right). With the mixed sample as a rough background estimate a straight-line fit gives an endpoint at $640 \mathrm{GeV}$ with a 
systematic shift of $1 \mathrm{GeV}$ from varying the bin size and fit range. The statistical error is $5 \mathrm{GeV}$. If, however, the same fitting procedure is applied to the $m_{q_{\mathrm{both}} l l}$ distribution, the endpoint value increases to $655 \pm 2 \mathrm{GeV}$ and the statistical error is $9 \mathrm{GeV}$.

Surely more study would bring these values closer, and optimally have them converge near the nominal value of $649.1 / 652.5 \mathrm{GeV}$ for $\tilde{u}_{L} / \tilde{d}_{L}$. For later use we take an optimistic statistical error of $5 \mathrm{GeV}$, but also include a systematic fit error of $3 \mathrm{GeV}$ for a more conservative estimate.

$\boldsymbol{m}_{\boldsymbol{q l}(\text { low })}$ : The $m_{\left.q_{\text {low }} l \text { low }\right)}$ distribution is shown in Fig. 14 (middle left). Both a consistency cut $m_{q_{\text {low }} l l}<670 \mathrm{GeV}$ and an inconsistency cut $m_{q_{\text {high }} l l}>670 \mathrm{GeV}$ are used. A straightline fit with no background hypothesis gives a statistical error around $6.3 \mathrm{GeV}$. The actual fit value is $443 \mathrm{GeV}$, which overshoots by a few $\mathrm{GeV}$ since the background plateau has not been included. The nominal value is $436.6 / 438.9 \mathrm{GeV}$ for $\tilde{u}_{L} / \tilde{d}_{L}$.

$\boldsymbol{m}_{\boldsymbol{q} \boldsymbol{l}(\text { high })}$ : The $m_{q_{\mathrm{low}} l(\text { high })}$ distribution with the consistency and the inconsistency cut is shown in Fig. 14 (middle right). The mixed sample was again used to roughly model the background under the edge. A straight-line fit gives an average value of $520.5 \mathrm{GeV}$ with systematics from binning and fit range of $3 \mathrm{GeV}$. The statistical error is at $5.5 \mathrm{GeV}$.

The nominal value is $529.9 / 532.7 \mathrm{GeV}$ for $\tilde{u}_{L} / \tilde{d}_{L}$, some $10 \mathrm{GeV}$ above our estimate. One could argue that the current endpoint measurement has considerable uncertainties and that this discrepancy is not dramatic at the present level of detail. However, such an underestimation is actually to be expected. In Fig. 10 the theoretical $m_{q l(\text { high }}$ distribution is shown for $\tilde{u}_{L}$ at $(\beta)$. There is a long vertical fall towards $517 \mathrm{GeV}$ (for $\tilde{d}_{L}$ it is at $519 \mathrm{GeV}$ ), then just before the bottom is reached, a small foot appears, as anticipated in Sect. 4.1, and takes us up by $11-13 \mathrm{GeV}$. To detect such a small foot would require more statistics than is available at $(\beta)$. Experimentally it is therefore expected to get an endpoint near 517-519 GeV. The incorrect endpoint measurement will have important consequences for the determination of masses from the endpoints. This situation is further discussed in Section 6 .

$\boldsymbol{m}_{\boldsymbol{q l l}\left(\boldsymbol{\theta}>\frac{\pi}{2}\right)}$ : The $m_{q l l\left(\theta>\frac{\pi}{2}\right)}$ distribution is shown in Fig. 14 (bottom). The same-flavour ('SF') and different-flavour ('DF') distributions are shown in solid and dashed black. Clearly, there are large uncertainties from the different-flavour subtraction. The statistical uncertainty of the different-flavour-subtracted sample was estimated with a straight-line fit, giving $13 \mathrm{GeV}$. In addition there will be a systematic error, here conservatively set to $10 \mathrm{GeV}$.

\section{Endpoint measurement values}

The results of the endpoint estimation for $(\alpha)$ and $(\beta)$ are summarized in Table $⿴$. The last column contains an estimate of the systematic error from different fitting techniques, ranges and bin widths. These values are not used in the following, but are included for completeness.

The column with the heading 'Energy Scale Error' shows the expected error on each endpoint estimation from the uncertainty on the absolute energy scale for jets and leptons. 


\begin{tabular}{|lccccc|}
\hline Edge & $\begin{array}{c}\text { Nominal } \\
\text { Value } \\
{[\mathrm{GeV}]}\end{array}$ & $\begin{array}{c}\text { Fit } \\
\text { Value } \\
{[\mathrm{GeV}]}\end{array}$ & $\begin{array}{c}\text { Energy Scale } \\
\text { Error }\left(\sigma^{\text {scale }}\right) \\
{[\mathrm{GeV}]}\end{array}$ & $\begin{array}{c}\text { Statistical } \\
\text { Error }\left(\sigma^{\text {stat }}\right) \\
{[\mathrm{GeV}]}\end{array}$ & $\begin{array}{c}\text { Syst. Fit } \\
\text { Error } \\
{[\mathrm{GeV}]}\end{array}$ \\
\hline$(\alpha)$ & & & & & \\
$m_{l l}^{\max }$ & 77.07 & 76.72 & 0.08 & 0.04 & 0.1 \\
$m_{q l l}^{\max }$ & 425.9 & 427.7 & 2.1 & 0.9 & 0.5 \\
$m_{q l(\text { low })}^{\max }$ & 298.5 & 300.7 & 1.5 & 0.9 & 0.5 \\
$m_{q l(\text { high })}^{\max }$ & 375.8 & 374.0 & 1.9 & 1.0 & 0.5 \\
$m_{q l l\left(\theta>\frac{\pi}{2}\right)}^{\min }$ & 200.7 & - & 1.0 & 2.2 & 2.0 \\
$m_{b l l\left(\theta>\frac{\pi}{2}\right)}^{\min }$ & 183.1 & - & 0.9 & 4.5 & 4.0 \\
\hline$(\beta)$ & & & & & 0.5 \\
$m_{l l}^{\max }$ & 137.9 & 137.4 & 0.14 & 5.0 & 3.0 \\
$m_{q l l}^{\max }$ & 649.1 & 647.0 & 3.2 & 6.3 & 4.0 \\
$m_{q l(\text { low }}^{\max }$ & 436.6 & 443.0 & 2.2 & 5.5 & 3.0 \\
$m_{q l(\text { high }}^{\max }$ & 529.9 & 520.5 & 2.6 & 13.0 & 10.0 \\
$m_{q l l\left(\theta>\frac{\pi}{2}\right)}^{\min }$ & 325.7 & - & 1.6 & & \\
\hline
\end{tabular}

Table 4: Endpoint values found from fitting the edges in Figs. 13 14, for $300 \mathrm{fb}^{-1}$. The nominal values correspond to the mass of $\tilde{u}_{L}$, which due to the proton content is produced at higher rates than the heavier $\tilde{d}_{L}$. For the thresholds no fit values are shown, only the errors. This reflects the fact that a reasonable fit function is lacking for this edge.

This effect has not been taken into account in the simulation. The uncertainties of the energy scales are here set to $1 \%$ for jets and $0.1 \%$ for electrons and muons, see Ch. 12 of [20]. For an invariant mass which consists of only jets or only leptons, this will give the same uncertainties, $1 \%$ and $0.1 \%$, respectively. If the invariant mass is constructed from one jet and one lepton, the endpoint uncertainty is

$$
\frac{\sigma\left(m_{q l}\right)}{m_{q l}}=\frac{\sigma\left(m_{q l}^{2}\right)}{2 m_{q l}^{2}}=\frac{1}{2} \sqrt{\left(\frac{\sigma\left(E_{j}\right)}{E_{j}}\right)^{2}+\left(\frac{\sigma\left(E_{l}\right)}{E_{l}}\right)^{2}}=0.50 \%
$$

where $E_{j}$ and $E_{l}$ are the jet and the lepton energies, respectively. For an invariant mass involving a higher number of jets and leptons, the error on the endpoint value from the energy scale uncertainty will be different for each event. The error of $m_{q l l}$ will depend on the relative size of the three terms on the right-hand side of Eq. (4.10). Since at $(\alpha)$ and $(\beta)$ we are in the region where the mass ratio $m_{\tilde{q}_{L}} / m_{\tilde{\chi}_{2}^{0}}$ dominates the two other mass ratios, see Eq. (4.4)-(1), the quark will usually be very energetic, leaving one or both $m_{q l}$ terms to dominate. This is particularly true at large values, so near the edge of $m_{q l l}$ one can show that the energy scale error will result in an endpoint error between $0.35 \%$ and $0.5 \%$ for each event. For $m_{q l l\left(\theta>\frac{\pi}{2}\right)}$ the average energy scale error will be slightly lower in our two scenarios. For simplicity we have set the energy scale error to $0.5 \%$ for all endpoints involving jets. 


\section{Extraction of masses from edges}

\subsection{0,000 ATLAS experiments}

In the simulation study described in Sect. . values for the endpoints and their statistical uncertainties were found, together with a 'systematic fit uncertainty'. Although not so far from the nominal endpoint values, the fit values in Table 4 are somewhat uncertain due to the as yet not-understood systematics of the fitting procedures. Also, the systematic error on the energy scale has not been addressed.

Assuming that one will eventually be able to control the systematics of the fitting, only the statistical errors together with the systematic error from the energy scale uncertainty will be what determine the LHC potential to measure the SUSY masses. To estimate this potential, consider an ensemble of typical LHC experiments, i.e. where the deviation of each endpoint measurement from the nominal value is based on a Gaussian distribution of width equal to the statistical error estimated for that endpoint, as well as a jet/lepton energy scale error picked from a Gaussian distribution for each experiment, in line with what is done in [31,

$$
E_{i}^{\text {exp }}=E_{i}^{\text {nom }}+A_{i} \sigma_{i}^{\text {stat }}+B \sigma_{i}^{\text {scale }}
$$

Here $E_{i}$ denotes the position of the $i^{\text {th }}$ endpoint. The coefficients $A$ and $B$ are picked from a Gaussian distribution of mean 0 and variance 1 . Each experiment will pick as many $A$ 's as there are endpoint measurements as well as one $B$ for the $m_{l l}$ endpoint and one for the endpoints involving jets, thus neglecting the effect of the lepton energy scale error on the latter.

When a set of edges $\mathbf{E}^{\text {exp }}$ has been found, the task is to find the masses $\hat{\mathbf{m}}$ which best correspond to the measurements. If only four endpoints are measured, the inversion formulae straight away return the possible mass combinations. If more endpoints are available, no mass combination will in general reproduce the edge measurements, and a numerical approach is required, where the measurements are weighted according to their uncertainties. Note that in this procedure we do not make use of the fit values given in Table 4.

It should also be emphasised that the systematics of the endpoint measurements are here assumed to be under control, i.e. the 'Syst. Fit Error' of Table 1 is neglected. The precision we will find in this section and the next for the determination of masses and mass differences at the LHC must be understood in this context. If the endpoint systematics turn out to be comparable to the combined statistical and energy scale errors, then the precision will be worse.

\subsection{Mass estimation via $\Sigma$}

In our case, where the jet energy scale error produces a correlation between the endpoint measurements, the method of least squares is appropriate. The best mass estimate $\hat{\mathbf{m}}$ is then the one which minimises the function

$$
\Sigma=\left[\mathbf{E}^{\mathrm{exp}}-\mathbf{E}^{\mathrm{th}}(\mathbf{m})\right]^{T} \mathbf{W}\left[\mathbf{E}^{\exp }-\mathbf{E}^{\mathrm{th}}(\mathbf{m})\right]
$$


where $\mathbf{E}^{\text {th }}(\mathbf{m})$ contains the theoretical edge values for a set of masses $\mathbf{m}$. The weight matrix $\mathbf{W}$ is the inverse of the correlation matrix or error matrix of the observations, which is given by the variances and covariances of the endpoint measurements,

$$
\begin{aligned}
& \left(\mathbf{W}^{-1}\right)_{i i}=\sigma_{i i}^{\text {stat }}+\sigma_{i i}^{\text {scale }}=\left(\sigma_{i}^{\text {stat }}\right)^{2}+\left(\sigma_{i}^{\text {scale }}\right)^{2} \\
& \left(\mathbf{W}^{-1}\right)_{i j}=\sigma_{i j}^{\text {scale }}=\left\langle E_{i}^{\exp } E_{j}^{\exp }\right\rangle-\left\langle E_{i}^{\exp }\right\rangle\left\langle E_{j}^{\exp }\right\rangle=\sigma_{i}^{\text {scale }} \sigma_{j}^{\text {scale }}, \quad i \neq j \\
& \left(\mathbf{W}^{-1}\right)_{i 1}=0, \quad i \neq 1
\end{aligned}
$$

where $j=1$ refers to $m_{l l}^{\max }$, which to a good approximation is uncorrelated with the other measurements. The covariances are similar to the variances in size, and so cannot be neglected. If the endpoint measurements were uncorrelated, $\mathbf{W}$ would become diagonal, and the least-squares method would reduce to the normal $\chi^{2}$ minimum method.

The ensemble distributions obtained by such a procedure can be interpreted as probability density functions. From these the 'inverse probability problem' can be addressed, which is that of stating something about the true masses on the basis of the ones obtained in one experiment. We will be interested in the mean values of the ensemble distribution, their standard deviations, skewness, as well as the correlation between masses.

\subsection{Minima of $\Sigma$}

Because many endpoints are given by different expressions for different mass regions, see Eqs (4.4)-(4.5), the minimisation function $\Sigma$ is a composite function. For the endpoint measurements used in this paper, $\Sigma$ is made up of nine individual functions, $\Sigma_{(i, j)}$, one for each of the nine regions $(i, j)$. Considered separately each function $\Sigma_{(i, j)}$ has one or more minima. For these to also be minima of the composite function ('physical minima'), they need to be situated in the region of validity ('home region') $(i, j)$ of the corresponding function. Physical minima can also occur on the borders between regions, in which case they will be referred to as 'border minima'.

If the threshold endpoints are left out, there are four measurements for four masses. The clear failure of the endpoint measurements of SPS 1a $(\alpha)$ and $(\beta)$ to comply with Eq. (4.11) already discards the three regions where these four measurements are not sufficient to determine the masses. In each of the other six regions the minima can be sought by use of the inversion formulae. Such solutions correspond to $\Sigma=0$. In cases where no physical solutions are found in this way, border minima exist at $\Sigma>0$, and will have to be found by a least square minimisation.

When the threshold measurement is included, the system of equations becomes overconstrained. This will give a non-uniform increase in the value of $\Sigma$, which may destroy or create minima. Another effect will be to move the minima of $\Sigma$ around in mass space, possibly moving them into or out of their home regions. One way to picture the effect is

to 'tune in' the new measurement by letting its uncertainty go from infinity, in which case the measurement has no effect, to the value specified in Table 1 . The masses and height of each $\Sigma$ minimum will then move continuously from the old to the new position.

Even though composite, $\Sigma$ is continuous, so its realisations in two neighbouring regions attain the same value at their common border. Assume that the endpoint measurements 
are such that one of the realisations has a minimum at the border (not a so-called border minimum). Consider first the case of no threshold measurement. Since $\Sigma=0$, also the other realisation must have a minimum at the border. If now one of the endpoint measurements is shifted up or down, the two minima will be driven off the border and also separated in mass space. To which side of the border they move, and whether they go to the same side or not, will depend on the actual parameters. If the minima are on the same side, as is the case at $(\beta)$, only one of them will be a physical minimum. If they are on opposite sides, as is the case at $(\alpha)$, either both will be physical minima, or neither, in which case there will be a border minimum.

This means that if a mass scenario is situated close to one of the borders, the endpoints it produces may also have been produced by a set of masses from the neighbouring region, provided the minimum there is a physical minimum.

If the threshold measurement is added, this picture is no longer exact. Since $\Sigma$ does not vanish at the minimum, the two regions will in general no longer have a common minimum at the border. However, the threshold measurement often has less weight than the other measurements, so the above picture still has some validity: Near a border two minima will be lurking. Both, one or none may be physical minima.

\subsection{SPS 1a $(\alpha)$}

If we neglect the threshold measurements to start with, and thereby also wait with the $\tilde{b}_{1}$ mass, there are for each 'experiment' two solutions. One solution is in region (1,1), which is the home region of the nominal masses, and one is in region (1,2). This is an example of the situation described above: SPS 1a $(\alpha)$ has $2 m_{\tilde{l}_{R}}^{2} /\left(m_{\tilde{\chi}_{1}^{0}}^{2}+m_{\tilde{\chi}_{2}^{0}}^{2}\right)=1.01$. If this ratio becomes less than one, the region changes, as is seen from Eq. 4.5). A reduction of $m_{\tilde{l}_{R}}$ by $0.7 \mathrm{GeV}$ would put the mass set on the border to region (1,2). In both regions the mass distributions are close to Gaussian. The ensemble means of the home region solution essentially equal the nominal values. The (1,2) solution has central values some $15-20 \mathrm{GeV}$ below the nominal ones. Without additional information both solutions are equally good and it would not be possible to determine which one to choose. One would have to state that the SUSY masses are summarized by either the $(1,1)$ set or by the $(1,2)$ set.

If the non- $b$ threshold endpoint is included as a fifth measurement in a least square minimisation, the situation changes. While a $(1,1)$ minimum exists for practically all the 'experiments', the occurence of a $(1,2)$ minimum is now slightly reduced to $85-90 \%$, but it is in the $\Sigma$ value of the minima the effect is most apparent. The overconstraining fifth measurement lifts the two minima asymmetrically from zero. As could be expected, the $(1,1)$ minimum is more often in accordance with the data, but there is always a nonnegligible $(1,2)$ contribution. The difference in $\Sigma$ value between a given minimum and the global minimum, $\Delta \Sigma$, is a measure of the relevance of the minimum. Table 5 shows how many minima are available, on average, for various $\Delta \Sigma$ cuts, and how these are shared between regions $(1,1)$ and $(1,2)$.

If only the lowest minimum is chosen, the wrong solution is returned in $10 \%$ of the experiments. However, if two minima exist and are close in $\Sigma$ value, one would have to consider both. In a certain fraction of experiments, depending on the $\Delta \Sigma$ cut, there 
would thus be two solution sets, e.g. for $\Delta \Sigma \leq 1$ we will have two solutions in $12 \%$ of the experiments. Whether or not it would be possible to select one of the solutions, and preferably the correct one, hinges on other measurements. In this case, where the masses of the two sets are quite close, they might be very difficult to distinguish, by e.g. cross-section considerations.

\begin{tabular}{|c|c|ccc|rrr|}
\hline & & \multicolumn{3}{|c|}{$(1,1)$} & \multicolumn{3}{|c|}{$(1,2)$} \\
& Nom & $\langle m\rangle$ & $\sigma$ & $\gamma_{1}$ & $\langle m\rangle$ & $\sigma$ & $\gamma_{1}$ \\
\hline$m_{\tilde{\chi}_{1}^{0}}$ & 96.1 & 96.3 & 3.8 & 0.2 & 85.3 & 3.4 & 0.1 \\
$m_{\tilde{l}_{R}}$ & 143.0 & 143.2 & 3.8 & 0.2 & 130.4 & 3.7 & 0.1 \\
$m_{\tilde{\chi}_{2}^{0}}$ & 176.8 & 177.0 & 3.7 & 0.2 & 165.5 & 3.4 & 0.1 \\
$m_{\tilde{q}_{L}}$ & 537.2 & 537.5 & 6.1 & 0.1 & 523.2 & 5.1 & 0.1 \\
$m_{\tilde{b}_{1}}$ & 491.9 & 492.4 & 13.4 & 0.0 & 469.6 & 13.3 & 0.1 \\
\hline$m_{\tilde{l}_{R}}-m_{\tilde{\chi}_{1}^{0}}$ & 46.92 & 46.93 & 0.28 & 0.0 & 45.08 & 0.72 & -0.2 \\
$m_{\tilde{\chi}_{2}^{0}}-m_{\tilde{\chi}_{1}^{0}}$ & 80.77 & 80.77 & 0.18 & 0.0 & 80.18 & 0.29 & -0.1 \\
$m_{\tilde{q}_{L}}-m_{\tilde{\chi}_{1}^{0}}$ & 441.2 & 441.3 & 3.1 & 0.0 & 438.0 & 2.7 & 0.0 \\
$m_{\tilde{b}_{1}}-m_{\tilde{\chi}_{1}^{0}}$ & 395.9 & 396.2 & 12.0 & 0.0 & 384.4 & 12.0 & 0.1 \\
\hline
\end{tabular}

Table 6: SPS 1a $(\alpha)$ : Minima for $\Delta \Sigma \leq 1$ in regions (1,1) and (1,2). Ensemble means, $\langle m\rangle$, and root-mean-square distances from the mean, $\sigma$, are in $\mathrm{GeV}$. The three lightest masses are very correlated. The mass of $\tilde{q}_{L}$ is fairly correlated to the lighter masses, but $m_{\tilde{b}_{1}}$ is essentially uncorrelated. The distributions are very close to symmetric.

The upper part of Table 6 shows the ensemble means of the masses, $\langle m\rangle$, the rootmean-square distances from the mean, $\sigma$, and skewness $\gamma_{1}^{4}$ of the two solutions for $\Delta \Sigma \leq 1$. The values are relatively stable with respect to the $\Delta \Sigma$ cut: The same table for $\Delta \Sigma \leq 99$ would for $(1,1)$ show a decrease in the masses by $0.1-0.2 \mathrm{GeV}$, and for $(1,2)$ an increase by

\begin{tabular}{|l|c|cc|}
\hline & \# Minima & $(1,1)$ & $(1,2)$ \\
\hline$\Delta \Sigma \leq 0$ & 1.00 & $90 \%$ & $10 \%$ \\
$\Delta \Sigma \leq 1$ & 1.12 & $94 \%$ & $17 \%$ \\
$\Delta \Sigma \leq 3$ & 1.30 & $97 \%$ & $33 \%$ \\
$\Delta \Sigma \leq 99$ & 1.88 & $99 \%$ & $88 \%$ \\
\hline
\end{tabular}

Table 5: Number of minima for various $\Delta \Sigma$ cuts and their whereabouts. $1-1.3 \mathrm{GeV}$.

The inclusion of the threshold measurement has very little effect on the ensemble values of the $(1,1)$ solution. For the $(1,2)$ solution, to better comply with the additional measurement, the masses have increased, and are now 10-15 GeV below the nominal ones. Also the $m_{\text {bll }}$ threshold was included in the fit which returned the values of Table 6. It is measured with much less precision than the other endpoints, so its inclusion has practically no effect on the other masses, only on $m_{\tilde{b}_{1}}$, for which it is the only measurement here.

The fact that the ensemble means of $(1,1)$ reproduce the nominal values, relates to the good average performance of the ensemble of experiments. The probability of doing well with only one experiment relies in addition on the spread of the ensemble values, given by $\sigma$. For SPS 1a $(\alpha)$ the high precision of the endpoint measurements translates into rather small $\sigma$ values. From the table we see e.g. that in $\sim 68 \%$ of the experiments the mass of $\tilde{\chi}_{1}^{0}$ from the $(1,1)$ solution will lie within $3.8 \mathrm{GeV}$ of the nominal value. The root-mean-square

${ }^{4}$ Skewness is defined by $\gamma_{1}=\mu_{3} /\left(\mu_{2}\right)^{3 / 2}=\mu_{3} / \sigma^{3}$, where $\mu_{i}=(x-\bar{x})^{i}$ is the $i^{\text {th }}$ moment about the mean. 


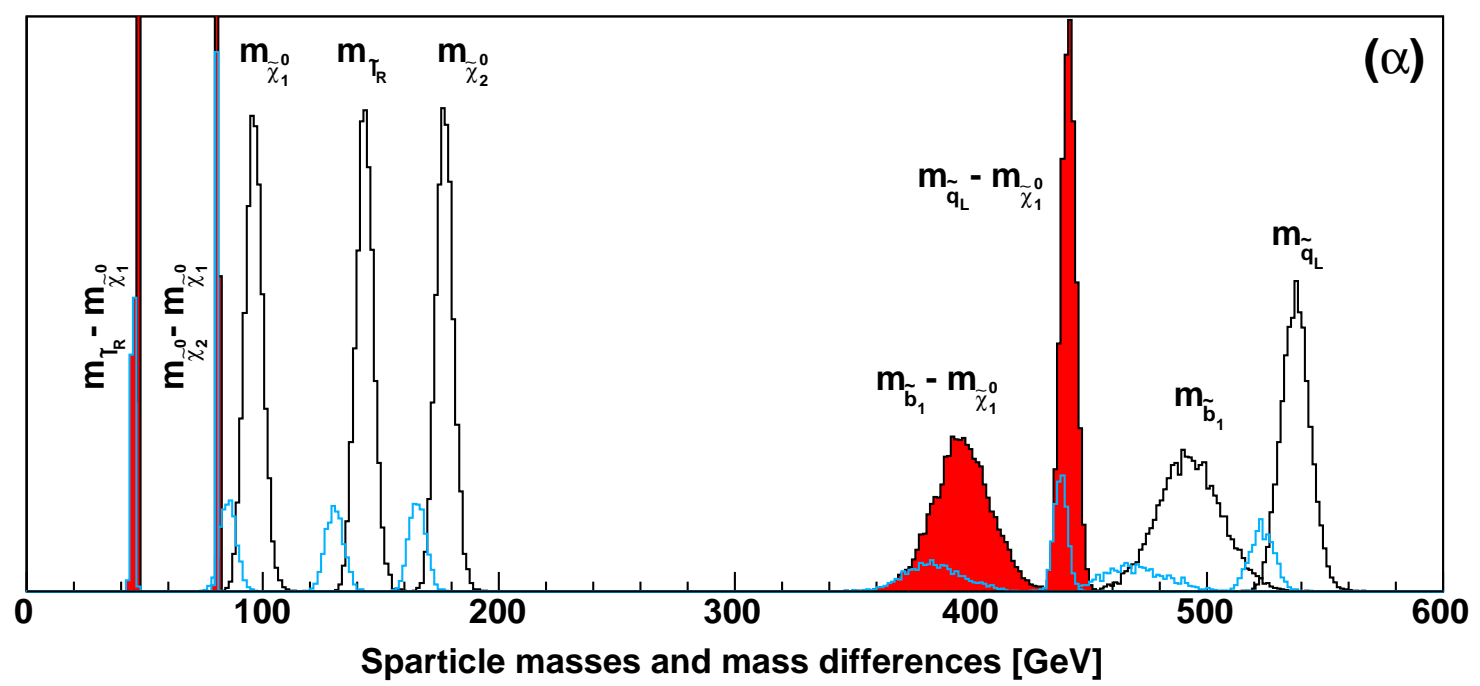

Figure 15: Sparticle masses and mass differences at SPS 1a $(\alpha)$ for solutions with $\Delta \Sigma \leq 1$. The unfilled distributions in black show from left to right $m_{\tilde{\chi}_{1}^{0}}, m_{\tilde{l}_{R}}, m_{\tilde{\chi}_{2}^{0}}, m_{\tilde{b}_{1}}$ and $m_{\tilde{q}_{L}}$ for solutions in the nominal region $(1,1)$. We will have such a solution in $94 \%$ of the experiments, see Table 5 . The unfilled distributions in blue show the same masses for solutions in region (1,2). Such a solution occurs in $17 \%$ of the experiments, and the masses returned are lower. The smaller rate of the $(1,2)$ solutions is reflected in the smaller area under the blue curves. The ratio of probabilities between $(1,2)$ and $(1,1)$ solutions is $17 \% / 94 \%=18 \%$. The area under one of the blue curves is $18 \%$ of the area under the corresponding black curve. The filled distributions show from left to right $m_{\tilde{l}_{R}}-m_{\tilde{\chi}_{1}^{0}}$, $m_{\tilde{\chi}_{2}^{0}}-m_{\tilde{\chi}_{1}^{0}}, m_{\tilde{b}_{1}}-m_{\tilde{\chi}_{1}^{0}}$ and $m_{\tilde{q}_{L}}-m_{\tilde{\chi}_{1}^{0}}$. Again, the most populated distributions (black curves) are for solutions in region $(1,1)$, the least populated (blue curves) for $(1,2)$ solutions. For mass differences there is more overlap between the $(1,1)$ and $(1,2)$ solutions, in particular for $m_{\tilde{l}_{R}}-m_{\tilde{\chi}_{1}^{0}}$ and $m_{\tilde{\chi}_{2}^{0}}-m_{\tilde{\chi}_{1}^{0}}$, of which only the lower parts of the distributions are visible. Mass differences are better determined than the masses themselves, reflected here by the narrower distributions of the former. The exception is $m_{\tilde{b}_{1}}$ which largely decouples from the other masses.

distances from the ensemble means are in principle unknown, as seen from one experiment. They can however be approximated by the procedure of simulating $10^{4}$ experiments, where the measured values play the role as 'nominal'. This will engender a systematic shift, but $\sigma$ and any skewness should be fairly well approximated. The root-mean-square distances from the mean values also have their counterparts in the $1 \sigma$ errors returned by the fit of each 'experiment'. To within a few percent they are found to be identical. This means that this information is available for the experiment actually performed. One can then make the inverse statement: For a given experiment one can with $\sim 68 \%$ confidence state that the nominal value of $m_{\tilde{\chi}_{1}^{0}}$ lies within $3.8 \mathrm{GeV}$ of the mass returned.

Due to the way masses enter in the endpoint expressions, the fit returns masses which have a strong positive correlation. If one mass is low at the minimum of the $\Sigma$ function, so the others tend to be and by a similar amount. In the lower part of Table 6 ensemble mean and root-mean-square values of mass differences are shown. It is clear that the three lightest sparticles are very correlated. Fix one and the others are given very accurately. The 
squark masses are less correlated. Also the results in region (1,2) are closer to $(1,1)$ and the nominal ones when considering mass differences. Fig. 15 shows the ensemble distributions corresponding to Table 6 .

Because of this strong correlation between the masses, not only the mean values and their $1 \sigma$ uncertainties, but the entire error matrix should be considered if one wants to use the result obtained by this method as input for other analyses.

A less involved solution would be to use less dependent variables, e.g. $m_{\tilde{\chi}_{1}^{0}}$ to set the scale, then differences for the remaining masses, $m_{\tilde{l}_{R}}-m_{\tilde{\chi}_{1}^{0}}, m_{\tilde{\chi}_{2}^{0}}-m_{\tilde{\chi}_{1}^{0}}, m_{\tilde{q}_{L}}-m_{\tilde{\chi}_{1}^{0}}$ and $m_{\tilde{b}_{1}}-m_{\tilde{\chi}_{1}^{0}}$.

Due to the high cross-section, most of the endpoints are determined with high precision, which in turn gives narrow and approximately symmetric ensemble distributions. The masses are thus determined with quite high precision. As a result of the strong correlations between in particular the lighter masses, even better estimates can be obtained for other combinations of the variables, e.g. mass differences. At SPS 1a $(\alpha)$ there is however a fair chance that two sets of masses do equally well in the minimisation procedure. Other considerations must in that case be made in order to choose between them, or both must be kept.

\subsection{SPS 1a $(\beta)$}

In combination with the theory plots of Section 4 we found in Section 5 that the $m_{q l(\mathrm{migh})}^{\max }$ value of $(\beta)$ would most probably on the average be underestimated by $11-13 \mathrm{GeV}$. We will find that this has a dramatic effect on the masses returned. However, for easier comparison with nominal values we first consider the situation without such a systematic effect. Then, afterwards, the impact of the mismeasurement will be shown, together with a way to mend the situation.

As in the previous case we start out without the threshold measurement. Also here two solutions are available for all 'experiments', one in $(1,1)$, the other in either $(1,2)$ or $(1,3)$. The nominal region for $(\beta)$ is $(1,2)$, but it is quite close to $(1,3)$. This becomes clear from an inspection of the 'border parameter',

$$
b=\frac{m_{\tilde{l}_{R}}^{2}}{m_{\tilde{\chi}_{1}^{0}} m_{\tilde{\chi}_{2}^{0}}},
$$

which is 1 on the border between these two regions. For $(\beta)$ we have $b=1.02$. If $m_{\tilde{l}_{R}}$ is reduced by $2.5 \mathrm{GeV}$, this ratio becomes unity and the the mass set sits on the border, see Eq. (4.5). The other point, $(\alpha)$, was near the border between $(1,1)$ and (1,2). There, both solutions (or none) were available. Here, the derivatives of $\Sigma$ are such that only one of the two solutions is available. In $71 \%$ of the cases we get $(1,2)$, in $29 \%$ we get $(1,3)$. While the 'low-mass' solution, (1,2) or (1,3), is in the vicinity of the nominal masses, the $(1,1)$ solution, which is always present, usually sits at much higher masses, $\left\langle m_{\tilde{\chi}_{1}^{0}}\right\rangle=514 \mathrm{GeV}$. Because the two solutions are so separated one may hope that the incorrect one will be sufficiently disfavoured by other measurements, e.g. cross-sections, that it can be discarded.

For $(\alpha)$ the solution in the nominal region on the average reproduced the nominal values to within $0.2-0.5 \mathrm{GeV}$. Here the $(1,2) /(1,3)$ solution has a mean of $m_{\tilde{\chi}_{1}^{0}}$ at $183 \mathrm{GeV}$, 
some $22 \mathrm{GeV}$ above the nominal value. The most probable value of the ensemble distribution is much closer to the nominal value. The distributions are infested with considerable skewness. On the way from endpoint measurement to mass determination a systematic effect which favours higher masses has been introduced. In statistical language our estimators of the true masses are not consistent: they do not converge to the nominal values. This of course has implications for the interpretation of the masses we obtain. What can be said about the true masses on the basis of the measured ones? We will return to the reasons for the skewness later.

When the threshold measurement is included, the (1,1) minimum usually yields a large $\Sigma$ value. Only in a small fraction of the experiments does it challenge the other minima. In the other sector there is either one minimum, positioned in (1,2), (1,3) or on the border (B), or there are two minima, in $(1,2)$ and $(1,3)$. These minima are usually in good agreement with the threshold measurement and have low $\Sigma$ values.

\begin{tabular}{|l|c|c|ccc|c|}
\hline & & & \multicolumn{3}{|c|}{1 sol } & 2 sol \\
& \# Min & $(1,1)$ & $(1,2)$ & $(1,3)$ & B & $(1,2) \&(1,3)$ \\
\hline$\Delta \Sigma \leq 0$ & 1.0 & $3 \%$ & $60 \%$ & $25 \%$ & $12 \%$ & $0 \%$ \\
$\Delta \Sigma \leq 1$ & 1.2 & $5 \%$ & $52 \%$ & $18 \%$ & $12 \%$ & $16 \%$ \\
$\Delta \Sigma \leq 3$ & 1.4 & $13 \%$ & $46 \%$ & $14 \%$ & $12 \%$ & $28 \%$ \\
$\Delta \Sigma \leq 99$ & 2.3 & $99 \%$ & $41 \%$ & $13 \%$ & $12 \%$ & $34 \%$ \\
\hline
\end{tabular}

Table 7: SPS 1a $(\beta)$ : Average number of minima and the fraction of experiments with the specified solution types, for different $\Delta \Sigma$ cuts.

Table 7 shows the average number of minima for different $\Sigma$ cuts. The three rightmost sections show the fraction of experiments which have the specified solution type. The two rightmost sections exclude one another. Either there is one solution in the low-mass sector, or there are two. For the one-solution case the whereabouts of the minimum is also shown. The home region of the nominal masses, (1,2), is seen to dominate. As it may well be possible to discard the $(1,1)$ minimum on the basis of other observations, it is logically separated from the low-mass minima. E.g. in $13 \%$ of the cases, regardless of the low-mass solution type, there is a $(1,1)$ minimum at $\Delta \Sigma \leq 3$. To get the average number of minima shown in column 2, sum horizontally, adding twice the two-solution percentage. For small $\Delta \Sigma$ cuts the two rightmost sectors do not add up to 1 . This simply means that in some cases the global minimum lies in $(1,1)$, and no low-mass minimum is available in the given $\Delta \Sigma$ range. For the current set of endpoint measurements the $(1,1)$ contamination is seen to be very moderate. However, the systematic fit error (column 6 of Table 1 ) is here assumed to be zero. If it should become impossible to obtain the threshold value with such optimistic precision, the fraction of $(1,1)$ solutions at low $\Sigma$ will grow rapidly.

In Table $8\langle m\rangle, \sigma$ and $\gamma_{1}$ of the ensemble masses and mass differences are shown for the different solution types and the cut $\Delta \Sigma \leq 1$. The masses of the $(1,1)$ solution are much higher than what the low-mass minima give. Even though the distributions are broad, allowing for low values to occur, it is very rare that the masses stretch down to the lowmass sector. In section 4 of the table the low-mass one-solution values are shown. Since 


\begin{tabular}{|c|c|c|c|c|c|c|c|c|c|c|c|c|c|}
\hline & & & & \multirow{2}{*}{\multicolumn{3}{|c|}{$\begin{array}{c}1 \text { solution } \\
(1,2) /(1,3) / \mathrm{B} \\
\end{array}$}} & \multicolumn{6}{|c|}{2 solutions } \\
\hline & & \multicolumn{3}{|c|}{$(1,1)$} & & & & \multicolumn{3}{|c|}{$(1,2)$} & \multicolumn{3}{|c|}{$(1,3)$} \\
\hline & $\mathrm{om}$ & $\langle m\rangle$ & $\sigma$ & $\gamma_{1}$ & $\langle m\rangle$ & $\sigma$ & $\gamma_{1}$ & $\langle m\rangle$ & $\sigma$ & $\gamma_{1}$ & $\langle m\rangle$ & $\sigma$ & $\gamma_{1}$ \\
\hline$\tilde{\chi}_{1}^{0}$ & 161 & 438 & 88 & 0.9 & 175 & 35 & 1.0 & 161 & 22 & 0.3 & 166 & 27 & 0.6 \\
\hline$\tilde{l}_{R}$ & 222 & 518 & 85 & 0.7 & 236 & 37 & 0.8 & 221 & 24 & 0.3 & 223 & 28 & 0.5 \\
\hline$\tilde{\chi}_{2}^{0}$ & 299 & 579 & 85 & 0.7 & 313 & 35 & 1.0 & 299 & 22 & 0.3 & 304 & 27 & 0.6 \\
\hline$\tilde{q}_{L}$ & 826 & 1146 & 104 & 0.8 & 843 & 44 & 0.9 & 826 & 30 & 0.3 & 835 & 36 & 0.5 \\
\hline$\tilde{l}_{R}-\tilde{\chi}_{1}^{0}$ & 61 & 81 & 1.8 & -0.3 & 61 & 4.4 & 0.4 & 61 & 1.9 & -0.2 & 57 & 1.3 & -0.2 \\
\hline$\tilde{\chi}_{2}^{0}-\tilde{\chi}_{1}^{0}$ & 138 & 141 & 0.9 & 0.1 & 138 & 0.6 & 0.2 & 138 & 0.5 & 0.0 & 138 & 0.5 & 0.0 \\
\hline$\tilde{q}_{L}-\tilde{\chi}_{1}^{0}$ & 665 & 708 & 17 & 0.1 & 668 & 10 & 0.5 & 665 & 9 & 0.1 & 669 & 10 & 0.2 \\
\hline
\end{tabular}

Table 8: SPS 1a $(\beta)$ : Nominal masses ('Nom') and $\Delta \Sigma \leq 1$ ensemble distribution values for the three solution types. High-mass sector: The $(1,1)$ solutions return masses far beyond the nominal values. Low-mass sector: For the one-solution case the values are based on the common distribution of $(1,2),(1,3)$ and border (B) solutions. In the two-solution case the ensemble variables of both solutions are shown. Ensemble means, $\langle m\rangle$, and root-mean-square values, $\sigma$, are in GeV.

in such a case only one acceptable solution is available (discarding $(1,1)$ ), and since $(\beta)$ anyway is situated close to the border, it makes sense to show the combined distribution of the (1,2), (1,3) and border (B) minima. From Table 7 this situation is seen to occur in $52 \%+18 \%+12 \%=82 \%$ of the experiments. The mean values of the masses lie some $15 \mathrm{GeV}$ above the nominal ones. This is an improvement compared to the non-threshold situation, but it remains an undesirable feature. The mass distributions are skewed and the most probable value is found close to the nominal value. The root-mean-square values of the ensemble distributions are large, nearly an order of magnitude larger than at $(\alpha)$.

The rightmost sections show the values for the two-solution type. The ensemble means of the two distributions do not differ too much, and they are much closer to the nominal values than is the case for the one-solution type. Since the values are rather close, it will probably be quite difficult to find other measurements which favours one of the sets. On the other hand, since the root-mean-square values are larger than the differences between the two solutions (also within one experiment), taking the average value, perhaps somehow weighted with the $\Sigma$ value, might be a possible compromise.

Again, the mass correlation is very strong. This is evident from the lower part of Table 8, where the ensemble distributions of mass differences come with much smaller root-mean-square distance to the mean values than what the masses themselves do. They are also very close to the nominal values. Even more seems to be gained by using mass differences here than at $(\alpha)$. Fig. 16 shows the ensemble distributions for the masses of all solutions with $\Delta \Sigma \leq 1$ and which lie in the regions $(1,2),(1,3)$ or on their common border. See figure caption for details.

\section{Skewness}

The ensemble distributions are not symmetric. While the most probable values are close to the nominal values, the means lie above. For $(\alpha)$ the tendency of such an asymmetry is 


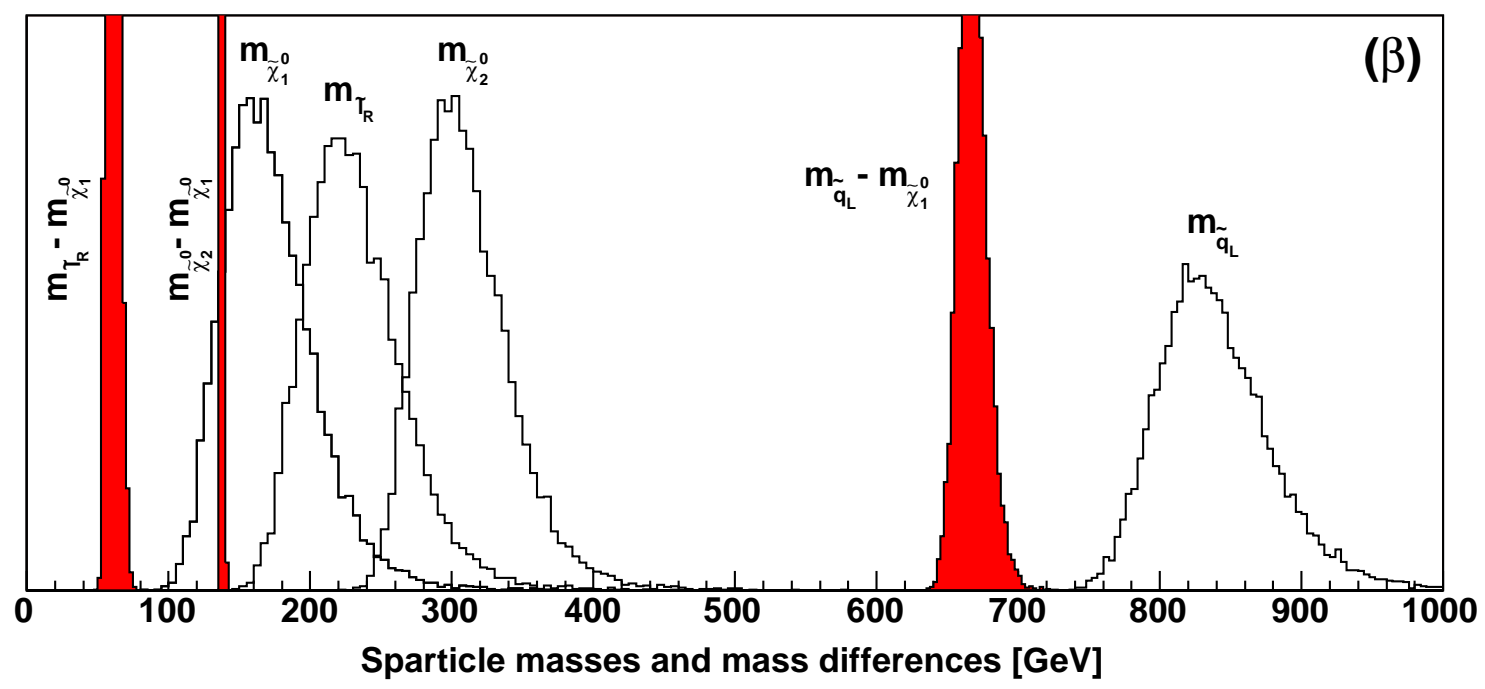

Figure 16: Sparticle masses and mass differences at SPS 1a $(\beta)$. All masses of solutions with $\Delta \Sigma \leq 1$ which lie in regions $(1,2),(1,3)$ and on their common border are shown. From left to right the unfilled distributions show $m_{\tilde{\chi}_{1}^{0}}, m_{\tilde{l}_{R}}, m_{\tilde{\chi}_{2}^{0}}$ and $m_{\tilde{q}_{L}}$. The filled distributions show the narrower mass differences $m_{\tilde{l}_{R}}-m_{\tilde{\chi}_{1}^{0}}, m_{\tilde{\chi}_{2}^{0}}-m_{\tilde{\chi}_{1}^{0}}$ and $m_{\tilde{q}_{L}}-m_{\tilde{\chi}_{1}^{0}}$. Skewness of mass distributions is visible.

small, but for $(\beta)$ the effect is large. The reason why we naively would expect a symmetric distribution around the nominal masses in the first place, is that the endpoint measurements are generated symmetrically. For complex functions like Eqs. (4.13)-(4.36) symmetric fluctuation of the endpoint arguments will produce near-symmetric variation of the function only for small fluctuations. As the arguments fluctuate more, the deviation from symmetry in the function values grows. At $(\alpha)$ the endpoint fluctuations are so small that the effect is negligible. For $(\beta)$, where the endpoint fluctuations are larger, the effect of the 'asymmetric propagation' is a noticeable increase of $3-4 \mathrm{GeV}$ for the ensemble means.

This is however not sufficient to explain the low-mass $\left\langle m_{\tilde{\chi}_{1}^{0}}\right\rangle$ of 183 and $173 \mathrm{GeV}$ $(\Delta \Sigma \leq 99)$ without and with the threshold measurement, respectively. 'Border effects' need to be considered. As described earlier, $(\beta)$ lies in $(1,2)$ but close to the border to $(1,3)$. First consider the situation without the threshold measurement. There is then always only one low-mass solution. If the (1,2) solution is physical, i.e. lies in (1,2), then the true minimum of $\Sigma_{(1,3)}$ also lies in region (1,2) and so is unphysical, and vice versa, as described in Sect. 6.3.

In Fig. 17 the mass of $\tilde{\chi}_{1}^{0}$ is plotted as a function of the border parameter, $b$, of Eq. (6.4), for both physical and unphysical minima of $\Sigma_{(1,2)}$ and $\Sigma_{(1,3)}$. The minima of $\Sigma_{(1,2)}$ are shown in red, from upper right to lower left. The $\Sigma_{(1,3)}$ solutions are in blue. Filled boxes are physical solutions, i.e. $\Sigma_{(1,2)}$ (red) for $b>1$ and $\Sigma_{(1,3)}$ (blue) for $b<1$. Empty boxes are unphysical solutions. An asymmetry arises from the accidental fact that for both functions the lower masses tend to lie in the unphysical region. The average of the entire $\Sigma_{(1,2)}$ distribution, both physical and unphysical minima, returns $164 \mathrm{GeV}$, the nominal value plus the $3 \mathrm{GeV}$ of the asymmetric propagation effect. It is then obvious that 


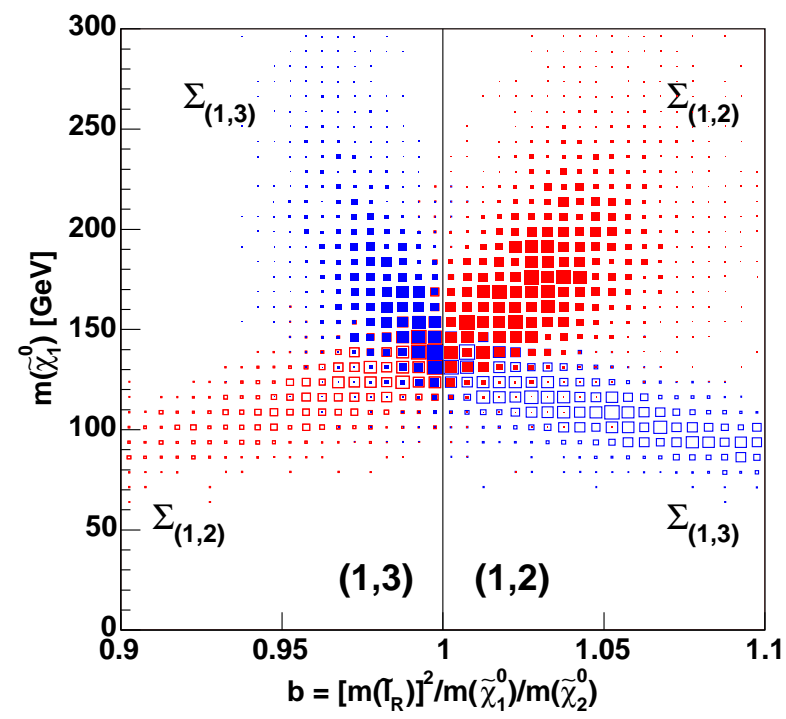

Figure 17: Border effect between region (1,2) and (1,3); the mass of $\tilde{\chi}_{1}^{0}$ as a function of the border parameter $b$, see Eq. (6.4), demonstrating border effects. Filled boxes represent physical solutions, empty boxes represent unphysical solutions.

when the unphysical $(1,2)$ solutions are replaced by the physical $(1,3)$ solutions which lie at higher masses, the ensemble mean increases. Here, this effect brings the average value for $m_{\tilde{\chi}_{1}^{0}}$ to $183 \mathrm{GeV}$, an additional increase of nearly $20 \mathrm{GeV}$.

When the threshold measurement is added, the border effect is reduced, giving an average value of $173 \mathrm{GeV}$ (for one solution). This is because a measurement will, unless there is any bias, on the average be conservative. It will try to keep the masses at their nominal values. Here, as the border effect is pushing the masses upwards, away from the nominal values, the threshold is holding back.

If, in a realistic situation, a set of endpoints has been measured, and the resulting set of masses is found to lie close to a border, caution should be exercised. Ad hoc procedures seem necessary for such a case. At least one is in a position to be aware of the danger. It is probably appropriate to consider unphysical minima as well.

\section{Mismeasured $m_{q l(\text { high })}^{\max }$}

As pointed out at the start of this subsection it is very likely that, for $(\beta), m_{q l \text { (high) }}^{\max }$ will be understimated by 11-13 GeV. Without the threshold measurement, the effect of this as returned by the inversion formulae, is an increase of $50 \mathrm{GeV}$ for the three lightest masses and $60 \mathrm{GeV}$ for the squark! If the threshold measurement is included, the effect is reduced to 30-40 GeV for the lighter masses, depending on the solution type, and 40-55 GeV for the squark. The increase is still surprisingly large and represents a serious threat to the applicability of the method in the case where the nominal masses happen to sit near a border. (Not to be confused with the previously mentioned border effect.) 
It may however be possible to discover afterwards that such a mismeasurement has or may well have been made. If the masses obtained are used to generate the corresponding theory distributions, one may spot the problem. A quick test showed that in our case the $m_{q l \text { (high) }}$ theory distribution as generated with the obtained masses, did have a small foot. This is then a sign that the original $m_{q l(\text { high })}^{\max }$ measurement may have been wrong, since no such foot was seen and included in the fit. This test probably has quite wide applicability. The reason is that even though, as we have seen, the masses may change a lot in the case of a mismeasured $m_{q l(\text { high })}^{\max }$, mass differences are not so far off the nominal values. The main change is therefore in the overall scale. For theory distributions, the shapes are independent of the overall scale. Only mass ratios are relevant. The theory shapes based on the masses obtained from the partly mismeasured endpoints, will therefore usually not deviate too much from the nominal shapes.

If one is certain that a mismeasurement has been made, either from the test described above or from other considerations, not only does one know that the $m_{q l(\text { high })}^{\max }$ measurement is not to be trusted. There is also a way to pursue the matter further. It relies on the fact that the vertical fall we see and measure, is the endpoint of the $m_{q l_{n}}$ distribution. We have the explicit expression for this endpoint, and so we can just replace the $m_{q l(\text { high }}^{\max }$ expression and redo the least square minimisation.

At SPS 1a $(\beta)$ many new aspects of the endpoint method have emerged. For a large fraction of the experiments a total of three minima were competing for a given set of endpoint values, some returning masses near the nominal values, some returning much higher masses. A generic characteristic is the skewness of the ensemble distributions. The problems and the causes of the asymmetry have been discussed, but a way to handle the asymmetry has not been proposed. The ensemble distributions are much broader than at SPS 1a $(\alpha)$. Mass differences are however quite precisely determined. The problem of mismeasuring $m_{q l \text { (high) }}^{\max }$, which will be a danger in a large fraction of mass space, has been discussed and a solution proposed. Throughout, we have also seen that the inclusion of a fifth measurement in general improves on the results.

\section{Linear Collider inputs}

As compared with the LHC, the Linear Collider 48, 49] will more directly provide very precise measurements of the lightest neutralino mass (and possibly also the second lightest). Thus, the LC input fixes the scale, whereas the cascade decays primarily provide mass differences. This kind of input, if it becomes available during the analysis of LHC data, will have a dramatic influence on the over-all analysis [27].

To each LHC experiment a corresponding Linear Collider experiment is considered. The Linear Collider measurement of the LSP, $m_{\tilde{\chi}_{1}^{0}}^{\mathrm{LC}}$, was for every experiment picked randomly from a Gaussian distribution of mean equal to $m_{\tilde{\chi}_{1}^{0}}^{\text {nom }}$, and standard deviation set by the expected uncertainty, $\sigma_{m_{\tilde{\chi}_{1}^{0}}}^{\mathrm{LC}}$. We used $\sigma_{m_{\tilde{\chi}_{1}^{0}}^{\mathrm{LC}}}^{\mathrm{L}}=0.05 \mathrm{GeV}$, in accordance with 48. Such a small uncertainty practically means fixing the mass at the nominal value. Still, for 
completeness the measurement was appropriately included in the least square minimisation by adding the term $\left[\left(m_{\tilde{\chi}_{1}^{0}}-m_{\tilde{\chi}_{1}^{0}}^{\mathrm{LC}}\right) / \sigma_{m_{\tilde{\chi}_{1}^{0}}}^{\mathrm{LC}}\right]^{2}$ to the $\Sigma$ function, Eq. (6.2).

\begin{tabular}{|l|c|ccc|c|}
\hline & & \multicolumn{3}{|c|}{$1 \mathrm{sol}$} & 2 sol \\
& \# Min & $(1,2)$ & $(1,3)$ & B & $(1,2) \&(1,3)$ \\
\hline$\Delta \Sigma \leq 0$ & 1.0 & $65 \%$ & $26 \%$ & $9 \%$ & $0 \%$ \\
$\Delta \Sigma \leq 1$ & 1.2 & $52 \%$ & $18 \%$ & $9 \%$ & $21 \%$ \\
$\Delta \Sigma \leq 3$ & 1.4 & $36 \%$ & $10 \%$ & $9 \%$ & $45 \%$ \\
$\Delta \Sigma \leq 99$ & 1.6 & $24 \%$ & $7 \%$ & $9 \%$ & $59 \%$ \\
\hline
\end{tabular}

Table 9: Number of minima for SPS 1a $(\beta)$ with LC input.

For $(\alpha)$ the fixing of $m_{\tilde{\chi}_{1}^{0}}$ reduces the occurrences of multiple minima to the per mille level for any usable minima, $\Delta \Sigma \leq 3$. In nearly all cases, $98-99 \%$, it is the home region $(1,1)$ minimum which survives. This seems reasonable since without the LC measurement the (1,2) minimum has $\left\langle m_{\tilde{\chi}_{1}^{0}}\right\rangle$ at some $10 \mathrm{GeV}$ below the nominal value, see Table 6 .

At $(\beta)$ the high-mass minimum $(1,1)$ is absent, disfavoured as it is by the Linear Collider measurement. The number of $(1,3)$ minima has increased, which is reflected in an increase of the two-solution case, compare Tables 7 and 9. For small $\Delta \Sigma$ cuts

\begin{tabular}{|c|r|rc|}
\hline & & \multicolumn{2}{|c|}{$(1,1)$} \\
& Nom & \multicolumn{1}{|c|}{$\langle m\rangle$} & \multicolumn{1}{c|}{$\sigma$} \\
\hline$\tilde{\chi}_{1}^{0}$ & 96.05 & 96.05 & 0.05 \\
$\tilde{l}_{R}$ & 142.97 & 142.97 & 0.29 \\
$\tilde{\chi}_{2}^{0}$ & 176.82 & 176.82 & 0.17 \\
$\tilde{q}_{L}$ & 537.25 & 537.2 & 2.5 \\
$\tilde{b}_{1}$ & 491.92 & 492.1 & 11.7 \\
\hline
\end{tabular}

Table 10: The SPS 1a $(\alpha)$ masses with LC input. All values in GeV. Region $(1,2)$ solutions now occur only in $\sim 1 \%$ of the cases and are left out. this increase is not drastic. Fixing $m_{\tilde{\chi}_{1}^{0}}$ thus does not help us to uniquely determine one minimum, contrary to what one might have expected.

The Tables 1011 show the mean and root-mean-square values of the ensemble distributions at $(\alpha)$ and $(\beta)$. For both SUSY scenarios the ensemble means fall very close to the nominal masses, even for minima not situated in the nominal home region. The uncertainty on the scale, which for the LHC alone is the main contribution to the spread of the ensemble distributions, is set to zero by the LC measurement. The root-mean-square values are therefore strongly reduced. Without Linear Collider measurements, the mass differences were more accurately determined in that they were less dependent on the mass scale. A comparison of the root-mean-square values of the masses in Tables 10 11 with the root-mean-square values of the mass differences in Tables 6 8 shows to which extent mass differences are scale independent variables. When a Linear Collider measurement is available, the mass differences no longer out-perform the masses themselves in terms of precision. With the fixing of the scale also the skewness of the distributions has vanished.

\section{Conclusions}

We have investigated the measurement of supersymmetric masses from the decay chain $\tilde{q} \rightarrow \tilde{\chi}_{2}^{0} q \rightarrow \tilde{l}_{R} l q \rightarrow \tilde{\chi}_{1}^{0} l l q$, in the Snowmass mSUGRA scenario SPS 1a. Since the lightest 


\begin{tabular}{|c|c|cc|cc|cc|}
\hline & & \multicolumn{2}{|c|}{1 solution } & \multicolumn{3}{|c|}{2 solutions } \\
\cline { 3 - 8 } & & \multicolumn{2}{|c|}{$(1,2) /(1,3) / \mathrm{B}$} & \multicolumn{2}{|c|}{$(1,2)$} & \multicolumn{2}{|c|}{$(1,3)$} \\
\hline & Nom & \multicolumn{1}{|c|}{$\langle m\rangle$} & $\sigma$ & \multicolumn{1}{|c|}{$\langle m\rangle$} & $\sigma$ & \multicolumn{1}{|c|}{$\langle m\rangle$} & $\sigma$ \\
\hline$\tilde{\chi}_{1}^{0}$ & 161.02 & 161.02 & 0.05 & 161.02 & 0.05 & 161.02 & 0.05 \\
$\tilde{l}_{R}$ & 221.86 & 221.15 & 3.26 & 222.22 & 1.32 & 217.48 & 1.01 \\
$\tilde{\chi}_{2}^{0}$ & 299.05 & 299.15 & 0.57 & 299.11 & 0.53 & 299.05 & 0.52 \\
$\tilde{q}_{L}$ & 826.29 & 826.1 & 6.3 & 825.9 & 5.8 & 828.6 & 5.5 \\
\hline
\end{tabular}

Table 11: The SPS 1a $(\beta)$ masses with LC input. Nominal, ensemble averages, $\langle m\rangle$, and rootmean-square deviations from the mean, $\sigma$, are all in $\mathrm{GeV}$.

neutralino $\tilde{\chi}_{1}^{0}$ is the LSP in most interesting mSUGRA scenarios, it will escape detection and only the quark and two leptons are available for the construction of invariant mass distributions. Nevertheless, the kinematic endpoints of these distributions have a well defined dependence on the masses of the particles in the decay chain and their measurement allows the extraction of the masses either by analytic inversion or numerical fit. The analytic expressions for the endpoints in terms of the masses were confirmed and presented together with their analytic inversions.

In order to measure the endpoints of the invariant mass distributions pertaining to the chosen decay chain, one must have the correct mass hierarchies for the decay chain and a large enough cross-section. To ensure that this decay chain could be used over a wide range of scenarios, we performed a scan over the SUSY parameters. We found that as long as $m_{0}$ was not too large in comparison to $m_{1 / 2}$, a large proportion of the allowed parameter space would display the correct mass hierarchy. Furthermore, on examination of the sparticle production cross-sections and decay branching ratios we found that a large cross-section for the decay was available over much of this region. The Snowmass mSUGRA SPS 1a line/point falls into this region and is a good candidate for study. However, we noted that the cross-section for the decay chain is particularly high for the SPS 1a point, and it is instructive to examine a second point on the SPS 1a line with a less optimistic cross-section. We have denoted this new point as SPS 1a $(\beta)$ while the original point became SPS 1a $(\alpha)$.

The LHC measurements of the endpoints were simulated using PYTHIA and ATLFAST. Hard kinematic cuts remove practically all Standard Model backgrounds, except $t \bar{t}$. Up to statistical fluctuations the powerful different-flavour subtraction then cancels the remaining $t \bar{t}$ as well as any lepton-uncorrelated background from other SUSY channels. The resulting distributions are however contaminated by lepton-correlated background from $\tilde{\chi}_{2}^{0}$ 's not taking part in the decay chain under study and combinatorial background from choosing the wrong jet. The inconsistency cut was shown to address the latter part with great efficiency, giving distributions much closer to the theoretical ones. Also mixed events were studied, revealing their potential to describe the background. More study is however needed.

The endpoints were found by simplistic fitting of the edges, usually with a straight line together with a reasonable background estimate. A Gaussian smearing was sometimes included as a first approximation of the various smearing effects which take place. The 
statistical precision of the edge was sought rather than an accurate determination of the endpoint. Still, the endpoints were seen to be in reasonably good agreement with the nominal values. However, the fitting procedure is clearly an area for improvement. On one hand, more realistic study of how the detector affects the distributions, and in particular the end regions, is called for. On the other hand, further study of the many realisations of the theory curves seems necessary. It is important to find good fit functions for the signal. A central part of such a programme is the incorporation of multiple squark masses at different rates. At a less ambitious scale the theory distributions should be studied for sheer acquaintance. The importance of such an awareness was demonstrated for the $m_{q l(\text { high })}^{\max }$ measurement at $(\beta)$.

In order to turn the endpoint measurements into particle masses, and understand the resulting errors of this procedure, we considered an ensemble of 10,000 'gedanken' experiments. For each experiment a numerical fit for the particle masses was performed, using the method of least squares, thus appropriately handling the correlation between measurements due to the common jet energy scale error. Where available the analytic expressions for the masses in terms of the endpoints were used to provide starting points for the fits.

The least squares function was found to often have two or even three minima of comparable importance, a consequence of the multiple realisations for many of the endpoints. Without the threshold measurement there are for both scenarios usually two equally good minima, one in the correct region and one in another region, giving different masses. When the threshold endpoint is added, the minimum in the correct region is usually preferred. Still, in a noticeable fraction of experiments there will be more than one solution. Due to less precise measurements this applies more to $(\beta)$ than to $(\alpha)$.

At $(\alpha)$ the minima of the correct region give masses very close to the nominal ones. The other (incorrect) region gives masses some 10-15 GeV lower. The ensemble distributions are symmetric. At $(\beta)$ there is one high-mass solution. The more precisely the threshold endpoint is determined, the less important this false minimum becomes. The low-mass solutions, one or two, are closer to the nominal values, but the distributions are skewed. This is a combined effect of the large endpoint uncertainties and the so-called border effect.

The obtained masses of the three lightest particles are found to be very strongly related. Furthermore it was seen that mass differences are better variables, in the sense that they are less correlated than the masses themselves. Due to the form of the endpoint expressions, the LHC will measure mass differences at high precision, but leave the overall scale less certain. A Linear Collider measurement of the LSP mass effectively sets the scale, which is why the precision of the masses improve drastically when the LHC and the Linear Collider measurements are combined.

\section{Acknowledgments}

This work has been performed partly within the ATLAS Collaboration, and we thank collaboration members for helpful discussions. We have made use of the physics analysis framework and tools which are the result of collaboration-wide efforts. It is a great 
pleasure to thank Giacomo Polesello for his contributions in the early stages of this work, and continued interest and advice. BKG would like to thank Steinar Stapnes for useful discussions. DJM would like to thank Ben Allanach for useful discussions. This research has been supported in part by the Research Council of Norway.

\section{References}

[1] S. Weinberg, Phys. Rev. D 13 (1976) 974; Phys. Rev. D 19 (1979) 1277; L. Susskind, Phys. Rev. D 20 (1979) 2619; G. 't Hooft, in Recent developments in gauge theories, Proceedings of the NATO Advanced Summer Institute, Cargese 1979, ed. G. 't Hooft et al. (Plenum, New York 1980).

[2] P. Fayet and S. Ferrara, Phys. Rept. 32 (1977) 249.

[3] S. Dimopoulos and H. Georgi, Nucl. Phys. B 193 (1981) 150.

[4] H. P. Nilles, Phys. Rept. 110 (1984) 1.

[5] H. E. Haber and G. L. Kane, Phys. Rept. 117 (1985) 75.

[6] R. Haag, J. T. Lopuszanski and M. Sohnius, Nucl. Phys. B 88 (1975) 257.

[7] M. B. Green, J. H. Schwarz and E. Witten, "Superstring Theory. Vol. 1: Introduction," Cambridge University Press (1987) (Cambridge Monographs On Mathematical Physics).

[8] N. Sakai and T. Yanagida, Nucl. Phys. B 197 (1982) 533.

[9] K. Inoue, A. Kakuto, H. Komatsu and S. Takeshita, Prog. Theor. Phys. 68 (1982) 927 [Erratum-ibid. 70 (1983) 330]; Prog. Theor. Phys. 71 (1984) 413.

[10] N. K. Falck, Z. Phys. C 30 (1986) 247.

[11] L. E. Ibanez and G. G. Ross, Phys. Lett. B 110 (1982) 215.

[12] L. E. Ibanez, Phys. Lett. B 118 (1982) 73.

[13] J. R. Ellis, D. V. Nanopoulos and K. Tamvakis, Phys. Lett. B 121 (1983) 123.

[14] L. Alvarez-Gaume, J. Polchinski and M. B. Wise, Nucl. Phys. B 221 (1983) 495.

[15] H. Goldberg, Phys. Rev. Lett. 50 (1983) 1419;

L. M. Krauss, Nucl. Phys. B 227 (1983) 556;

J. R. Ellis, J. S. Hagelin, D. V. Nanopoulos, K. A. Olive and M. Srednicki, Nucl. Phys. B 238 (1984) 453.

[16] F. Gianotti, New J. Phys. 4 (2002) 63.

[17] B. C. Allanach, G. A. Blair, S. Kraml, H. U. Martyn, G. Polesello, W. Porod and P. M. Zerwas, arXiv:hep-ph/0403133.

[18] A. H. Chamseddine, R. Arnowitt and P. Nath, Phys. Rev. Lett. 49 (1982) 970.

[19] V. M. Lobashev et al., Phys. Lett. B 460 (1999) 227.

[20] ATLAS Collaboration, ATLAS Detector and Physics Performance Technical Design Report 2. No. CERN-LHCC-99-014 ATLAS-TDR-14. May, 1999.

[21] B. C. Allanach et al., in Proc. of the APS/DPF/DPB Summer Study on the Future of Particle Physics (Snowmass 2001) ed. N. Graf, Eur. Phys. J. C 25 (2002) 113 [eConf C010630 (2001) P125] [arXiv:hep-ph/0202233]. 
[22] H. Baer, C. h. Chen, F. Paige and X. Tata, Phys. Rev. D 53 (1996) 6241 [arXiv:hep-ph/9512383].

[23] I. Hinchliffe, F. E. Paige, M. D. Shapiro, J. Soderqvist and W. Yao, Phys. Rev. D 55 (1997) 5520 [arXiv:hep-ph/9610544].

[24] I. Hinchliffe, F. E. Paige, E. Nagy, M. D. Shapiro, J. Soderqvist and W. Yao, LBNL-40954

[25] H. Bachacou, I. Hinchliffe and F. E. Paige, Phys. Rev. D 62 (2000) 015009 [arXiv:hep-ph/9907518].

[26] G. Polesello, Precision SUSY measurements with ATLAS for SUGRA point 5, ATLAS Internal Note, PHYS-No-111, October 1997.

[27] G. Weiglein et al. [LHC / ILC Study Group], arXiv:hep-ph/0410364.

[28] B. K. Gjelsten, E. Lytken, D. J. Miller, P. Osland, G. Polesello, A detailed analysis of the measurement of SUSY masses with the ATLAS detector at the LHC, ATL-PHYS-2004-007, Geneva, CERN, Jan 2004.

[29] S. Eidelman et al. [Particle Data Group Collaboration], Phys. Lett. B 592 (2004) 1.

[30] H. Baer, F. E. Paige, S. D. Protopopescu and X. Tata, arXiv:hep-ph/9305342, arXiv:hep-ph/0001086.

[31] B. C. Allanach, C. G. Lester, M. A. Parker and B. R. Webber, JHEP 0009 (2000) 004 [arXiv:hep-ph/0007009].

[32] C. G. Lester, Model independent sparticle mass measurements at ATLAS, Ph. D. thesis, http://www.slac.stanford.edu/spires/find/hep/www?r=cern-thesis-2004-003

[33] B. K. Gjelsten, D. J. Miller, P. Osland, arXiv:hep-ph/0501033

[34] J. R. Ellis, K. A. Olive and Y. Santoso, New J. Phys. 4 (2002) 32 [arXiv:hep-ph/0202110].

[35] J. R. Ellis, K. A. Olive, Y. Santoso and V. C. Spanos, Phys. Lett. B 565 (2003) 176 [arXiv:hep-ph/0303043].

[36] A. Raklev, presented at "Nordic LHC Workshop", Copenhagen, June 2004, http://hep.nbi.dk/NLHC9-NG7/

[37] J. A. Casas, A. Lleyda and C. Munoz, Nucl. Phys. B 471 (1996) 3 [arXiv:hep-ph/9507294]; S. Abel and T. Falk, Phys. Lett. B 444 (1998) 427 [arXiv:hep-ph/9810297]; D. G. Cerdeno, E. Gabrielli, M. E. Gomez and C. Munoz, JHEP 0306 (2003) 030 [arXiv:hep-ph/0304115].

[38] B. C. Allanach, in Proc. of the APS/DPF/DPB Summer Study on the Future of Particle Physics (Snowmass 2001) ed. N. Graf, eConf C010630 (2001) P319 [arXiv:hep-ph/0110227].

[39] C. L. Bennett et al., Astrophys. J. Suppl. 148 (2003) 1 [arXiv:astro-ph/0302207].

[40] D. N. Spergel et al., Astrophys. J. Suppl. 148 (2003) 175 [arXiv:astro-ph/0302209].

[41] M. M. Nojiri, D. Toya and T. Kobayashi, Phys. Rev. D 62 (2000) 075009 [arXiv:hep-ph/0001267].

[42] B. K. Gjelsten, Ph. D. thesis, University of Oslo, 2004.

[43] P. Richardson, JHEP 0111 (2001) 029 [arXiv:hep-ph/0110108]; A. J. Barr, arXiv:hep-ph/0405052. 
[44] T. Sjöstrand, P. Edén, C. Friberg, L. Lönnblad, G. Miu, S. Mrenna, E. Norrbin, Comput. Phys. Commun. 135 (2001) 238; T. Sjostrand, L. Lonnblad and S. Mrenna, "PYTHIA 6.2: Physics and manual", arXiv:hep-ph/0108264.

[45] H. L. Lai et al. [CTEQ Collaboration], Eur. Phys. J. C 12 (2000) 375 [arXiv:hep-ph/9903282].

[46] G. Marchesini, B. R. Webber, G. Abbiendi, I. G. Knowles, M. H. Seymour and L. Stanco, Comput. Phys. Commun. 67 (1992) 465;

G. Corcella, I.G. Knowles, G. Marchesini, S. Moretti, K. Odagiri, P. Richardson, M. H. Seymour, B. R. Webber, JHEP 0101 (2001) 010 [arXiv:hep-ph/9912396]

[47] E. Richter-Was, D. Froidevaux and L. Poggioli, "ATLFAST 2.0: a fast simulation package for ATLAS", Tech. Rep. ATL-PHYS-98-131 (1998)

[48] J. A. Aguilar-Saavedra et al. [ECFA/DESY LC Physics Working Group Collaboration], "TESLA Technical Design Report Part III: Physics at an e+e- Linear Collider," arXiv:hep-ph/0106315.

[49] T. Abe et al. [American Linear Collider Working Group Collaboration], "Linear collider physics resource book for Snowmass 2001. 1: Introduction," in Proc. of the APS/DPF/DPB Summer Study on the Future of Particle Physics (Snowmass 2001) ed. N. Graf, SLAC-R-570, arXiv:hep-ex/0106055. 